\title{
THE ALPERIN WEIGHT CONJECTURE AND UNO'S CONJECTURE FOR THE BABY MONSTER B,$p$ ODD
}

\author{
JIANBEI AN AND R. A. WILSON
}

\begin{abstract}
Suppose that $p$ is 3,5 or 7 . In this paper, faithful permutation representations of maximal $p$-local subgroups are constructed, and the radical $p$-chains of the Baby Monster $\mathbb{B}$ are classified. Hence, the Alperin weight conjecture and the Uno reductive conjecture can be verified for $\mathbb{B}$, the latter being a refinement of Dade's reductive conjecture and the Isaacs-Navarro conjecture.
\end{abstract}

\section{Introduction}

Recently, Isaacs and Navarro [12] proposed a new conjecture that is a refinement of the Alperin-McKay conjecture, and Uno [15] raised an alternating sum version of the conjecture that is a refinement of the Dade conjecture [9].

Dade's reductive conjecture [9] has been verified for all of the sporadic simple groups except $\mathrm{Fi}_{24}^{\prime}, \mathbb{B}$ and $\mathbb{M}$. The use of computer algebra systems, (namely MAGMA [6] and GAP [10]) to study permutation (or in some cases matrix) representations of the groups has been a central step of the program. Since the smallest faithful permutation representation of $\mathbb{B}$ has degree 13571955000 , it is difficult to verify the conjecture directly. However, from the classification [16] of maximal $p$-local subgroups of $\mathbb{B}$, we know that when $p$ is equal to 3,5 or 7 , the normalizer of each radical $p$-subgroup of $\mathbb{B}$ is a subgroup of one of precisely thirteen maximal $p$-local subgroups. Thus we can classify radical chains in these maximal subgroups without performing any calculation in $\mathbb{B}$.

In this paper, we construct a faithful permutation representation for each maximal $p$-local subgroup. We then classify radical chains, and hence verify the Alperin weight conjecture and Uno's refinement of Dade's reductive conjecture for $\mathbb{B}$.

The paper is organized as follows. In Section 2, we fix the notation, state the conjectures in detail, and state three lemmas. In Section 3, we explain how to construct faithful permutation representations of the thirteen maximal $p$-local subgroups. In Section 4 , we recall the modified local strategy $[4,5]$; we also explain how we applied it to determine the radical subgroups of each maximal subgroup, and how to determine the fusion of the radical subgroups in $\mathbb{B}$. In Section 5, we classify radical $p$-subgroups of $\mathbb{B}$, and verify the Alperin weight conjecture. In Section 6, we do some cancellations in the alternating sum of Uno's conjecture, and then determine radical chains (up to conjugacy) and their local structures. In the last section, we verify Uno's projective conjecture for $\mathbb{B}$.

The first author was supported by the Marsden Fund of New Zealand via grant \#9144/3368248.

Received 20 January 2004, revised 23 February 2004; published 11 May 2004.

2000 Mathematics Subject Classification 20C20, 20C34, 20 D08.

(C) 2004, Jianbei An and R. A. Wilson 


\section{Conjectures and lemmas}

Let $p$ be a prime and $R$ a $p$-subgroup of a finite group $G$. Then $R$ is radical if $O_{p}(N(R))=R$, where $O_{p}(N(R))$ is the largest normal $p$-subgroup of the normalizer $N(R)=N_{G}(R)$. Denote by $\operatorname{Irr}(G)$ the set of all irreducible ordinary characters of $G$, and let $\operatorname{Blk}(G)$ be the set of $p$-blocks, $B \in \operatorname{Blk}(G)$ and $\varphi \in \operatorname{Irr}(N(R) / R)$. The pair $(R, \varphi)$ is called a $B$-weight if $\mathrm{d}(\varphi)=0$ and $B(\varphi)^{G}=B$ (in the sense of Brauer), where $\mathrm{d}(\varphi)=\log _{p}\left(|G|_{p}\right)-\log _{p}\left(\varphi(1)_{p}\right)$ is the $p$-defect of $\varphi$ and $B(\varphi)$ is the block of $N(R)$ containing $\varphi$. A weight is always identified with its $G$-conjugates. Let $\mathcal{W}(B)$ be the number of $B$-weights, and let $\ell(B)$ be the number of irreducible Brauer characters of $B$. Alperin [1] conjectured that $\mathcal{W}(B)=\ell(B)$ for each $B \in \operatorname{Blk}(G)$.

Given a $p$-subgroup chain

$$
C: P_{0}<P_{1}<\ldots<P_{n}
$$

of $G$, define $|C|=n, C_{k}: P_{0}<P_{1}<\ldots<P_{k}$, and

$$
N(C)=N_{G}(C)=N\left(P_{0}\right) \cap N\left(P_{1}\right) \cap \ldots \cap N\left(P_{n}\right) .
$$

The chain $C$ is said to be radical if it satisfies the following two conditions:

(a) $P_{0}=O_{p}(G)$, and

(b) $P_{k}=O_{p}\left(N\left(C_{k}\right)\right)$ for $1 \leqslant k \leqslant n$.

Denote by $\mathcal{R}=\mathcal{R}(G)$ the set of all radical $p$-chains of $G$. Let $B \in \operatorname{Blk}(G)$, and let $D(B)$ be a defect group of $B$. The $p$-local rank (see [2]) of $B$ is the number

$$
\operatorname{plr}(B)=\max \left\{|C|: C \in \mathcal{R}, C: P_{0}<P_{1}<\ldots<P_{n} \leqslant D(B)\right\} .
$$

Let $Z$ be a cyclic group, $\hat{G}=Z . G$ a central extension of $Z$ by $G$, and $C \in \mathcal{R}(G)$. Denote by $N_{\hat{G}}(C)$ the preimage $\eta^{-1}(N(C))$ of $N(C)$ in $\hat{G}$, where $\eta$ is the natural group homomorphism from $\hat{G}$ onto $G$ with kernel $Z$. Let $\rho$ be a faithful linear character of $Z$, and let $\hat{B}$ be a block of $\hat{G}$ covering the block $B(\rho)$ of $Z$ containing $\rho$. Denote by $\operatorname{Irr}\left(N_{\hat{G}}(C), \hat{B}, d, \rho\right)$ the irreducible characters $\psi$ of $N_{\hat{G}}(C)$ such that $\psi$ lies over $\rho, d(\psi)=d$ and $B(\psi)^{\hat{G}}=\hat{B}$, and set $\mathrm{k}\left(N_{\hat{G}}(C), \hat{B}, d, \rho\right)=\left|\operatorname{Irr}\left(N_{\hat{G}}(C), \hat{B}, d, \rho\right)\right|$.

DADE's PROJECTIVE CONJECTURE (see [9]). If $O_{p}(G)=1$ and $\hat{B}$ is a p-block of $\hat{G}$ covering $B(\rho)$ with defect group $D(\hat{B}) \neq O_{p}(Z)$, then

$$
\sum_{C \in \mathcal{R} / G}(-1)^{|C|} \mathrm{k}\left(N_{\hat{G}}(C), \hat{B}, d, \rho\right)=0,
$$

where $\mathcal{R} / G$ is a set of representatives for the $G$-orbits of $\mathcal{R}$.

If $Z=1$, then $\hat{G}=G, \hat{B}=B$ and $\rho=1$. Set $\mathrm{k}(N(C), B, d)=\mathrm{k}\left(N_{\hat{G}}(C), \hat{B}, d, \rho\right)$. The projective conjecture is then called the ordinary conjecture.

DADE'S ORDINARY CONJECTURE (see [8]). If $O_{p}(G)=1$ and $B$ is a p-block of $G$ with defect group $D(B) \neq 1$, then for any integer $d \geqslant 0$,

$$
\sum_{C \in \mathcal{R} / G}(-1)^{|C|} \mathrm{k}(N(C), B, d)=0 .
$$


Let $\hat{H}$ be a subgroup of a finite group $\hat{G}$, let $\varphi \in \operatorname{Irr}(\hat{H})$ and let $r(\varphi)=r_{p}(\varphi)$ be the integer $0<r(\varphi) \leqslant(p-1)$ such that the $p^{\prime}$-part $(|\hat{H}| / \varphi(1))_{p^{\prime}}$ of $|\hat{H}| / \varphi(1)$ satisfies

$$
\left(\frac{|\hat{H}|}{\varphi(1)}\right)_{p^{\prime}} \equiv r(\varphi)(\bmod p) .
$$

Given an integer $1 \leqslant r \leqslant(p-1) / 2$, let $\operatorname{Irr}(\hat{H},[r])$ be the subset of $\operatorname{Irr}(\hat{H})$ consisting of characters $\varphi$ such that $r(\varphi) \equiv \pm r(\bmod p)$, and let

$$
\begin{gathered}
\operatorname{Irr}(\hat{H}, \hat{B}, d, \rho,[r])=\operatorname{Irr}(\hat{H}, \hat{B}, d, \rho) \cap \operatorname{Irr}(\hat{H},[r]) ; \\
\mathrm{k}(\hat{H}, \hat{B}, d, \rho,[r])=|\operatorname{Irr}(\hat{H}, \hat{B}, d, \rho,[r])| .
\end{gathered}
$$

Suppose that $Z=1$, and let $\hat{B}=B \in \operatorname{Blk}(G)$ with a defect group $D=D(B)$ and the Brauer correspondent $b \in \operatorname{Blk}\left(N_{G}(D)\right)$. Then $\mathrm{k}(N(D), B, d(B),[r])$ is the number of characters $\varphi \in \operatorname{Irr}(b)$ such that $\varphi$ has height 0 and $r(\varphi) \equiv \pm r(\bmod p)$, where $\mathrm{d}(B)$ is the defect of $B$.

IsAaCS-NAVARro CONJECTURE (see [12, Conjecture B]). In the notation above,

$$
\mathrm{k}(G, B, \mathrm{~d}(B),[r])=\mathrm{k}(N(D), B, \mathrm{~d}(B),[r]) .
$$

The following refinement of Dade's conjecture is due to Uno.

Uno's PRoJective CONJECTURE (see [15, Conjecture 3.2]). If $O_{p}(G)=1$ and if $D(\hat{B}) \neq$ $O_{p}(Z)$, then for any integer $d \geqslant 0$, faithful $\rho \in \operatorname{Irr}(Z)$ and $1 \leqslant r \leqslant(p-1) / 2$,

$$
\sum_{C \in \mathcal{R} / G}(-1)^{|C|} \mathrm{k}\left(N_{\hat{G}}(C), \hat{B}, d, \rho,[r]\right)=0 .
$$

Similarly, if $Z=1$, then the projective conjecture is the ordinary conjecture. Note also that if $p=2$ or $p=3$, then Uno's conjecture is equivalent to Dade's conjecture.

Let $G$ be the Baby Monster, $\mathbb{B}$. Then its Schur multiplier is cyclic of order 2, and its outer automorphism group is trivial, so Dade's projective conjecture is equivalent to his reductive conjecture (and Uno's reductive conjecture is also equivalent to his reductive conjecture). Thus it suffices to verify:

1. Dade's ordinary conjecture for $\mathbb{B}$;

2. Dade's projective conjecture for the 2 covering group $2 \cdot \mathbb{B}$ when $p=3$;

3. Uno's ordinary conjecture for $\mathbb{B}$; and

4. Uno's projective conjecture for $2 \cdot \mathbb{B}$ when $p \geqslant 5$.

The proofs of the following two lemmas are straightforward.

Lemma 2.1. Let $\sigma: O_{p}(G)<P_{1}<\ldots<P_{m-1}<Q=P_{m}<P_{m+1}<\ldots<P_{\ell}$ be $a$ fixed radical $p$-chain of a finite group $G$, where $1 \leqslant m<\ell$. Suppose that

$$
\sigma^{\prime}: O_{p}(G)<P_{1}<\ldots<P_{m-1}<P_{m+1}<\ldots<P_{\ell}
$$

is also a radical p-chain such that $N_{G}(\sigma)=N_{G}\left(\sigma^{\prime}\right)$. Let $\mathcal{R}^{-}(\sigma, Q)$ be the subfamily of $\mathcal{R}(G)$ consisting of chains $C$ whose $(\ell-1)$ th subchain, $C_{\ell-1}$, is conjugate to $\sigma^{\prime}$ in $G$. Let $\mathcal{R}^{0}(\sigma, Q)$ be the subfamily of $\mathcal{R}(G)$ consisting of chains $C$ whose $\ell$ th subchain $C_{\ell}$ is conjugate to $\sigma$ in $G$. Then the map $g$ sending any $O_{p}(G)<P_{1}<\ldots<P_{m-1}<P_{m+1}<$ $\ldots<P_{\ell}<\ldots$ in $\mathcal{R}^{-}(\sigma, Q)$ to $O_{p}(G)<P_{1}<\ldots<P_{m-1}<Q<P_{m+1}<\ldots<P_{\ell}<\ldots$ induces a bijection, denoted again by $g$, from $\mathcal{R}^{-}(\sigma, Q)$ onto $\mathcal{R}^{0}(\sigma, Q)$. Moreover, for any $C$ in $\mathcal{R}^{-}(\sigma, Q)$, we have $|C|=|g(C)|-1$ and $N_{G}(C)=N_{G}(g(C))$. 
Lemma 2.2. Suppose that $Q$ is a p-subgroup of $G$. Then $Q$ is radical in $G$ if and only if $N_{G}(Q) \leqslant M$ and $Q$ is radical in $M$ for some maximal p-local subgroup $M$ of $G$.

LEMмa 2.3. If $Q$ is a $p$-subgroup of a finite group $G$, then there is a radical $p$-subgroup $R$ such that

$$
Q \leqslant R \quad \text { and } \quad N_{G}(Q) \leqslant N_{G}(R) .
$$

Proof. This follows by [2, Lemma 2.1].

\section{Construction of permutation representations of maximal p-local subgroups}

We will follow the notation of [7]. In particular, if $p$ is odd, then $p^{1+2 \gamma}=p_{+}^{1+2 \gamma}$ is an extra-special group of order $p^{1+2 \gamma}$ with exponent $p$, and if $\delta=+$ or $\delta=-$, then $2_{\delta}^{1+2 \gamma}$ is an extra-special group of order $2^{1+2 \gamma}$ with type $\delta$. If $X$ and $Y$ are groups, we use $X . Y$, $X \cdot Y$ and $X: Y$ to denote an extension, a nonsplit extension and a split extension of $X$ by $Y$, respectively. Given a positive integer $n$, we use $p^{n}$ to denote the elementary abelian group of order $p^{n}, n$ to denote the cyclic group of order $n, D_{2 n}$ to denote the dihedral group of order $2 n$, and $S D_{2 n}$ to denote the semidihedral group of order $2 n$.

The subgroups of $2 \cdot \mathbb{B}$ that we need to construct are given in Table 1 .

The general strategy in most cases is to make appropriate subgroups of the Monster first, and to centralize a suitable involution to get the desired subgroup of $2 \cdot \mathbb{B}$. We can then quotient by the central involution to obtain the corresponding subgroup of $\mathbb{B}$.

Table 1: Maximal $p$-local subgroups of $\mathbb{B}$ and $2 \cdot \mathbb{B}$.

\begin{tabular}{|c|c|c|}
\hline Shape in $\mathbb{B}$ & Shape in $2 \cdot \mathbb{B}$ & Overgroup in $\mathbb{M}$ \\
\hline$\left(7: 3 \times 2 \cdot L_{3}(4) \cdot 2\right) .2$ & $\left(7: 3 \times 2^{2} \cdot L_{3}(4) \cdot 2\right) .2$ & $(7: 3 \times \mathrm{He}): 2$ \\
\hline$\left(2^{2} \times 7^{2}:\left(3 \times 2 A_{4}\right)\right) \cdot 2$ & $\left(D_{8} \times 7^{2}:\left(3 \times 2 A_{4}\right)\right) .2$ & $\left(L_{2}(7) \times 7^{2}:\left(3 \times 2 A_{4}\right)\right) .2$ \\
\hline $5: 4 \times \mathrm{HS}: 2$ & $\left(D_{10} \times 2 \cdot\right.$ HS.2).2 & $\left(D_{10} \times \mathrm{HN}\right) .2$ \\
\hline $5^{1+4}: 2_{-}^{1+4} \cdot A_{5} \cdot 4$ & $5^{1+4}: 2.2_{-}^{1+4} \cdot A_{5} \cdot 4$ & $5^{1+6}: 2 \cdot J_{2} \cdot 4$ \\
\hline $5^{2}: 4 S_{4} \times S_{5}$ & $\left(5^{2}: 4 A_{4} \times 2 \cdot S_{5}\right) .2$ & $\left(5^{2}: 4 \circ Q_{8} \times U_{3}(5)\right): S_{3}$ \\
\hline $5^{3} \cdot L_{3}(5)$ & $2 \times 5^{3} \cdot L_{3}(5)$ & $\mathrm{n} / \mathrm{a}$ \\
\hline$S_{3} \times \mathrm{Fi}_{22}: 2$ & $\left(S_{3} \times 2 \cdot \mathrm{Fi}_{22}\right) .2$ & $3 \cdot \mathrm{Fi}_{24}$ \\
\hline $3^{1+8}: 2_{-}^{1+6} \cdot U_{4}(2) .2$ & $3^{1+8}: 2.2_{-}^{1+6} \cdot U_{4}(2) .2$ & $3^{1+12} \cdot 2 \cdot \operatorname{Suz}: 2$ \\
\hline$\left(3^{2}: D_{8} \times U_{4}(3) \cdot 2^{2}\right) \cdot 2$ & $\left(3^{2}: 2 \times 2 \cdot U_{4}(3) \cdot 2^{2}\right) \cdot D_{8}$ & $\left(3^{2}: 2 \times O_{8}^{+}(3)\right) \cdot S_{4}$ \\
\hline $3^{2} \cdot 3^{3} \cdot 3^{6} \cdot\left(S_{4} \times 2 \cdot S_{4}\right)$ & $3^{2} \cdot 3^{3} \cdot 3^{6}:\left(2 \cdot S_{4} \times 2 \cdot S_{4}\right)$ & $3^{2} \cdot 3^{5} \cdot 3^{10}\left(M_{11} \times 2 \cdot S_{4}\right)$ \\
\hline $3^{3} \cdot 3 \cdot 3^{3} \cdot 3^{3}\left(L_{3}(3) \times 2\right)$ & $2 \times 3^{3} \cdot 3 \cdot 3^{3} \cdot 3^{3}\left(L_{3}(3) \times 2\right)$ & $2 \times \mathrm{Fi}_{23}$ \\
\hline $3^{3} \cdot 3^{6}\left(L_{3}(3) \times D_{8}\right)$ & $3^{3} \cdot 3^{6}\left(L_{3}(3) \times S D_{16}\right)$ & $\left(2 \times O_{8}^{+}(3)\right) \cdot S_{4}$ \\
\hline $3^{6}:\left(2 \times L_{4}(3)\right) \cdot 2^{2}$ & $2 \times 3^{6}:\left(L_{4}(3) \cdot S D_{16}\right)$ & $\left(2 \times O_{8}^{+}(3)\right) \cdot S_{4}$ \\
\hline
\end{tabular}


Many of the groups required are subdirect products, and are thus easy to construct from representations of the constituent groups. For example, to make $(7: 3 \times \mathrm{He}): 2$ we first make the Frobenius group 7:6 generated by the permutations $a=(1,2,3,4,5,6,7)$ and $b=(1,3,2,6,4,5)$, and He:2 generated by two permutations $c$ and $d$ on 2058 points. Since $a$ and $c$ are in the respective subgroups of index 2, while $b$ and $d$ are not, we see that $a c$ and $b d$ together generate the desired group $(7: 3 \times \mathrm{He}): 2$ acting on $7+2058=2065$ points. Inside this group, we then find the involution centralizer by standard methods, and obtain $\left(7: 3 \times 2^{2} \cdot L_{3}(4) .2\right) .2$ as the required subgroup of $2 \cdot \mathbb{B}$. Taking the quotient by the central involution, we obtain the corresponding subgroup $\left(7: 3 \times 2 \cdot L_{3}(4) .2\right) .2$ of $\mathbb{B}$.

Similarly, we construct the affine group $7^{2}:\left(3 \times 2 S_{4}\right)<7^{2}: \mathrm{GL}_{2}(7)$ as a permutation group on forty-nine points, and $L_{2}(7): 2$ as a permutation group on eight points. Thus we obtain the direct product acting on fifty-seven points, and its subgroup $\left(7^{2}:\left(3 \times 2 A_{4}\right) \times L_{2}(7)\right) .2$ of index 2 in the same way as above. By centralizing an involution in the $L_{2}(7)$ subgroup we obtain the required subgroup $\left(7^{2}:\left(3 \times 2 A_{4}\right) \times D_{8}\right) .2$ of $2 \cdot \mathbb{B}$, and its quotient $\left(7^{2}:\left(3 \times 2 A_{4}\right) \times 2^{2}\right) .2$ in $\mathbb{B}$. This completes the construction of the maximal 7-local subgroups of $\mathbb{B}$ and $2 \cdot \mathbb{B}$.

Next consider the 5-local subgroups. We can make $\left(D_{10} \times \mathrm{HN}\right) .2$ as a subdirect product on $5+1140000$ points, and we centralize an involution to get $\left(D_{10} \times 2 \cdot \mathrm{HS}: 2\right) .2$ and its quotient 5:4 $\times$ HS:2. However, these permutations are rather large, so we actually made the group directly as a subdirect product of $5: 4$ and $4 \cdot \mathrm{HS}: 2$. Note that there is an outer element of $\left(D_{10} \times 2 \cdot \mathrm{HS}: 2\right) \cdot 2$, which acts as the outer automorphism of $2 \cdot \mathrm{HS}: 2$ (multiplying elements outside $2 \cdot$ HS by the central involution) and squares to the product of an involution in the $D_{10}$ and the central involution of $2 \cdot \mathrm{HS}$.

Similarly, we make $5^{1+6}: 2 \cdot J_{2} .4$ by following the instructions in [14] for making groups of extraspecial type. Specifically, we make a matrix representation in eight dimensions over GF(5) in which a complementary $2 J_{2} .4$ and the normal $5^{1+6}$ are represented by matrices of shape

$$
\left(\begin{array}{lll}
\mu & 0 & 0 \\
0 & A & 0 \\
0 & 0 & 1
\end{array}\right) \text { and }\left(\begin{array}{ccc}
1 & 0 & 0 \\
-S v^{T} & I_{6} & 0 \\
\lambda & v & 1
\end{array}\right)
$$

respectively, where $A$ is a typical element of $2 \cdot J_{2} .4$ in its six-dimensional representation over $\mathbb{F}_{5}$, and $S$ is the matrix of the symplectic form preserved by $2 J_{2}$ in this representation (note that $S$ has inadvertently been replaced by $S^{-1}$ from the bottom of [14, p. 316]), and $\mu$ is the scalar such that $A S A^{T}=\mu S$. We can then convert to a permutation representation on the $5^{7}=78125$ images of the vector $(0,0,0,0,0,0,0,1)$. Centralizing a suitable involution leads to the corresponding subgroups $5^{1+4}: 2.2_{-}^{1+4} . A_{5} \cdot 4$ of $2 \cdot \mathbb{B}$ and $5^{1+4}: 2_{-}^{1+4} . A_{5} .4$ of $\mathbb{B}$.

The next subgroup can be made as a subdirect product of the affine group $5^{2}: 4 S_{4}<$ $5^{2}: \mathrm{GL}_{2}(5)$ (on twenty-five points) and the almost simple group $U_{3}(5): S_{3}$ (on 126 points) leading to $\left(5^{2}: 4 \circ Q_{8} \times U_{3}(5)\right): S_{3}$ on 151 points. We then centralize an involution to get $\left(5^{2}: 4 A_{4} \times 2 S_{5}\right) .2$ in $2 \cdot \mathbb{B}$ and $5^{2}: 4 S_{4} \times S_{5}$ in $\mathbb{B}$.

Finally for $p=5$, we find that $5^{3} \cdot L_{3}(5)$ is isomorphic to a subgroup of the Lyons group, for which generators are available [17] as $111 \times 111$ matrices over GF(5). This can be converted to a permutation representation on 7750 points, by permuting a suitable orbit of vectors in a suitable subquotient of this representation. As there is no double cover of $L_{3}(5)$, the corresponding subgroup of $2 \cdot \mathbb{B}$ is a direct product $2 \times 5^{3} \cdot L_{3}(5)$. This concludes the construction of the maximal 5-local subgroups. 
We next take $3 \cdot \mathrm{Fi}_{24}$ acting on $3 \times 306936$ points from [17], and centralize an involution to get $\left(S_{3} \times 2 \mathrm{Fi}_{22}\right)$.2. Its quotient $S_{3} \times \mathrm{Fi}_{22}: 2$ is a direct product; it is thus easily constructed as a permutation group on $3+3510$ points.

For the next group, we could take $3^{1+12}: 2 \cdot \mathrm{Suz}: 2$ as constructed in [14], and convert it into permutations on $3^{13}=1594323$ points. Although this is not a subgroup of the Monster, the involution centralizer $3^{1+8}: 2.2_{-}^{1+6} U_{4}(2) .2$ is isomorphic to the desired subgroup of $2 \cdot \mathbb{B}$. However, these permutations are rather large, so instead we take the involution centralizer $2.2_{-}^{1+6} U_{4}(2) .2$ in $2 \cdot$ Suz.2, acting on an 8 -space and a 4 -space over GF(3). We lift the action on the 8 -space to an action of $3^{1+8}: 2_{-}^{1+6} U_{4}(2) .2$ on a 10 -space, and thence to an action on $3^{9}=19683$ points. To get the double cover, we adjoin the permutation action on the eighty non-zero vectors of the 4-space.

We take $\left(3^{2}: 2 \times O_{8}^{+}(3)\right) . S_{4}$ as a subdirect product of the affine group $3^{2}: 2 S_{4}$ on nine points and $O_{8}^{+}(3): S_{4}$ on 3360 points, and then find the involution centralizer $\left(3^{2}: 2 \times 2 \cdot U_{4}(3) \cdot 2^{2}\right) \cdot D_{8}$ and its quotient $\left(3^{2}: D_{8} \times U_{4}(3) \cdot 2^{2}\right) \cdot 2$. Another involution centralizer $\left(2 \times O_{8}^{+}(3)\right) \cdot S_{4}$ contains the last two 3-locals in Table 1, corresponding to certain maximal parabolic subgroups in $O_{8}^{+}(3): S_{4}$. These are therefore straightforward to construct. The third last of the maximal 3-local subgroups listed in Table 1 is contained in $\mathrm{Fi}_{23}$, which becomes $2 \times \mathrm{Fi}_{23}$ in $2 \cdot \mathbb{B}$. It can therefore be constructed by using information on the character tables of maximal subgroups of $\mathrm{Fi}_{23}$, given in [10].

Finally, we need to construct $3^{2} \cdot 3^{3} \cdot 3^{6}\left(S_{4} \times 2 S_{4}\right)$ and its double cover. The latter is a subgroup of the group $3^{2} \cdot 3^{5} \cdot 3^{10}:\left(M_{11} \times 2 \cdot S_{4}\right)$ in the Monster, and so has shape $3^{2} \cdot 3^{3} \cdot 3^{6}:\left(2 \cdot S_{4} \times 2 \cdot S_{4}\right)$. We first make the corresponding subgroup of $\mathbb{B}$ by explicitly finding words in the standard generators which generate this subgroup. To do this, we start from $3^{1+8}: 2_{-}^{1+6} U_{4}(2) \cdot 2$, and find a subgroup $3^{2} \cdot\left[3^{7}\right] \cdot 2 \cdot 3^{3}\left(S_{4} \times 2\right)$, being the normalizer of a $3 B$-pure $3^{2}$ generated by the centre and one other element of $3^{1+8}$. We then go to work in the centralizer of an $S_{4}$ to find an element swapping the two generators for the $3^{2}$. First we centralize a suitable involution in the $S_{4}$, to get a group $\left(2^{2} \times F_{4}(2)\right): 2$, and then we centralize an element of order 4 squaring to this involution, to get a group $4 \times{ }^{2} F_{4}(2)$. In here, we find by random search, an involution that extends our $3^{2}$ to $A_{6}$, in which it is easy to find the involution that we are seeking. This involution now extends the group $3^{2} \cdot\left[3^{9}\right] .\left(S_{4} \times 2 \times S_{3}\right)$ to the maximal subgroup $3^{2} \cdot 3^{3} \cdot 3^{6} \cdot\left(S_{4} \times 2 \cdot S_{4}\right)$ of $\mathbb{B}$, which we are seeking.

To construct a suitable permutation representation, we find a subgroup of order $2^{5} .3^{5}$ centralizing an involution, and permute the $2^{2} \cdot 3^{8}=26244$ images of a fixed vector of this subgroup. To construct the double cover, observe that we need only to cover the quotient $S_{4}$, which we can do by taking a subdirect product with the group $2 \cdot S_{4} \cong \mathrm{GL}_{2}(3)$ acting on eight points.

\section{A local subgroup strategy and fusions}

From [16], we know that each radical $p$-subgroup $R$ of $\mathbb{B}$ is radical in one of the conjugates $M$ of maximal $p$-local subgroups constructed in Section 3 and, further, $N_{\mathbb{B}}(R)=N_{M}(R)$.

In [4] and [5], a (modified) local strategy was developed to classify the radical $p$ subgroups $R$. We review this method here. Suppose that $M$ is a subgroup of a finite group $G$ satisfying $N_{G}(R)=N_{M}(R)$.

Step (1) We first consider the case where $M$ is $p$-local. Let $Q=O_{p}(M)$, so that $Q \leqslant R$. Choose a subgroup $X$ of $M$. We explicitly compute the coset action of $M$ on the cosets of $X$ 
in $M$; we obtain a group $W$ representing this action, a group homomorphism $f$ from $M$ to $W$, and the kernel $K$ of $f$. For a suitable $X$, we have $K=Q$, and the degree of the action of $W$ on the cosets is much smaller than that of $M$. We can now directly classify the radical $p$-subgroup classes of $W$ (or apply Step (2) below to $W$ ), and the preimages in $M$ of the radical subgroup classes of $W$ are the radical subgroup classes of $M$.

Step (2) Now consider the case where $M$ is not $p$-local. We may be able to find its radical $p$-subgroup classes directly. Alternatively, we find a (maximal) subgroup $K$ of $M$ such that $N_{K}(R)=N_{M}(R)$ for each radical subgroup $R$ of $M$. If $K$ is $p$-local, then we apply Step (1) to $K$. If $K$ is not $p$-local, we can replace $M$ by $K$, and repeat Step (2).

Steps (1) and (2) constitute the modified local strategy. After applying the strategy, we list the radical subgroups of each $M$, and then do the fusions as follows.

Suppose that $R$ is a radical $p$-subgroup of $M$. Using the local structure, we can determine whether or not $N_{M}(R)$ is a subgroup of another maximal subgroup $M^{\prime}$. Suppose that $N_{M}(R)$ is a subgroup of $M^{\prime}$. By Lemma 2.3, there is a radical subgroup $R^{\prime}$ of $M^{\prime}$ such that $R \leqslant R^{\prime}$ and $N_{M}(R) \leqslant N_{M^{\prime}}\left(R^{\prime}\right)$. Using the local structure, we can determine whether or not $R$ is radical in $M^{\prime}$, and if so, we can identify $R$ with a radical subgroup $R^{\prime}$ of $M^{\prime}$. Some details are given in the proof of Proposition 5.1.

The computations reported in this paper were carried out using MAGMA V2.10-6 on a Sun UltraSPARC Enterprise 4000 server.

\section{Radical subgroups and weights}

Let $\mathcal{R}_{0}(G, p)$ be a set of representatives for conjugacy classes of radical $p$-subgroups of $G$. For $H, K \leqslant G$, we write $H \leqslant G K$ if $x^{-1} H x \leqslant K$, and we write $H \in_{G} \mathcal{R}_{0}(G, p)$ if $x^{-1} H x \in \mathcal{R}_{0}(G, p)$ for some $x \in G$.

Let $G$ be the Baby Monster $\mathbb{B}$. Then

$$
|G|=2^{41} \cdot 3^{13} \cdot 5^{6} \cdot 7^{2} \cdot 11 \cdot 13 \cdot 17 \cdot 19 \cdot 23 \cdot 31 \cdot 47,
$$

and we may suppose that $p \in\{2,3,5,7\}$, since both conjectures hold for a block with a cyclic defect group, by [8, Theorem 7.1] and [3, Theorem 5.2]. Suppose that $p$ is odd, so that $p=3,5,7$.

Denote by $\operatorname{Irr}^{0}(H)$ the set of ordinary irreducible characters of $p$-defect 0 of a finite group $H$, and by $\mathrm{d}(H)$ the number $\log _{p}\left(|H|_{p}\right)$. Given $R \in \mathcal{R}_{0}(G, p)$, let $C(R)=C_{G}(R)$, $N=N_{G}(R)$ and $\hat{N}=N_{2 \cdot \mathbb{B}}(R)$, where $R$ is viewed as a subgroup of the covering group 2.BB. If $B_{0}=B_{0}(G)$ is the principal $p$-block of $G$, then (see $\left.[4,(4.1)]\right)$

$$
\mathcal{W}\left(B_{0}\right)=\sum_{R}\left|\operatorname{Irr}^{0}(N / C(R) R)\right|,
$$

where $R$ runs over the set $\mathcal{R}_{0}(G, p)$ such that $\mathrm{d}(C(R) R / R)=0$. The character table of $N / C(R) R$ can be calculated by Magma, and so we find $\left|\operatorname{Irr}^{0}(N / C(R) R)\right|$. Write $\mathcal{W}_{N}=$ $\left|\operatorname{Irr}^{0}(N / C(R) R)\right|$ and $\mathcal{W}_{\hat{N}}=\left|\operatorname{Irr}^{0}\left(N_{2 . \mathbb{B}}(R) / C(R) R\right)\right|-\left|\operatorname{Irr}^{0}(N / C(R) R)\right|$; the latter will be used to calculate the number of weights for $2 . \mathbb{B}$.

Proposition 5.1. The non-trivial radical p-subgroups $R$ of $G=\mathbb{B}$ (up to conjugacy) and their local structures are given in Tables 2 and 3, according to whether $p \geqslant 5$ or $p=3$, where $S \in \mathrm{Syl}_{3}(G)$ is a Sylow 3-subgroup of $G$. 
Proof. Case (1) Suppose that $p=7$. By [16, Section 7], $\mathbb{B}$ has two non-trivial radical 7-subgroups, 7 and $7^{2}$, with local structures given by Table 2 .

Case (2) Suppose that $p=5$. By [16, Theorem 6.4], $\mathbb{B}$ has four maximal 5-local subgroups:

$$
\begin{array}{ll}
M_{1}=N(5 A)=5: 4 \times \mathrm{HS}: 2 ; & M_{2}=N\left(5 B^{3}\right)=5^{3} \cdot L_{3}(5) ; \\
M_{3}=N(5 B)=5_{+}^{1+4}: 2_{-}^{1+4} \cdot A_{5} \cdot 4 ; & M_{4}=N\left(5 A^{2}\right)=5^{2}: 4 S_{4} \times S_{5} .
\end{array}
$$

Let $M=M_{3}$ or $M=M_{4}$. Since $\left|M / O_{5}(M)\right|_{5}=5$, it follows that a Sylow subgroup of $M$ is its only radical 5-subgroup other than $O_{5}(M)$. Thus

$$
\mathcal{R}_{0}\left(M_{i}, 5\right)= \begin{cases}\left\{5^{2}, 5^{3}\right\} & \text { if } i=4 \\ \left\{5_{+}^{1+4}, 5_{+}^{1+4} .5\right\} & \text { if } i=3\end{cases}
$$

in addition, $N_{M_{4}}\left(5^{3}\right)=5^{2}: 4 S_{4} \times 5: 4$, and $N_{M_{3}}\left(5_{+}^{1+4} .5\right)=5_{+}^{1+4} \cdot 5.4^{2}$.

We may take

$$
\mathcal{R}_{0}\left(M_{1}, 5\right)=\left\{5,5^{2}, 5 \times 5_{+}^{1+2}\right\},
$$

where $5 \times 5_{+}^{1+2} \in \operatorname{Syl}_{5}\left(M_{1}\right)$. In addition,

$$
N_{M_{1}}(R)= \begin{cases}5: 4 \times 5: 4 \times S_{5} & \text { if } R=5^{2} \\ 5 \times 5_{+}^{1+2} 4 . D_{8} & \text { if } R=5 \times 5_{+}^{1+2}\end{cases}
$$

Note that $5^{2} \in \mathbb{B} \mathcal{R}_{0}\left(M_{4}, 5\right)$, so $N_{\mathbb{B}}(R) \neq N_{M_{1}}(R)$ for any $R \in \mathcal{R}_{0}\left(M_{1}, 5\right) \backslash\{5\}$.

We may take

$$
\mathcal{R}_{0}\left(M_{2}, 5\right)=\left\{5^{3}, 5_{+}^{1+4}, 5^{2} .5^{1+2}, 5_{+}^{1+4} .5\right\}
$$

in addition,

$$
\begin{aligned}
& C_{M_{2}}\left(5_{+}^{1+4}\right)=5=Z\left(5_{+}^{1+4}\right) \\
& N_{M_{2}}\left(5^{2} \cdot 5^{1+2}\right)=5^{2} \cdot 5^{1+2} \cdot \mathrm{GL}_{2}(5) ; \quad N_{M_{2}}\left(5_{+}^{1+4}\right)=5_{+}^{1+4} \cdot \mathrm{GL}_{2}(5)
\end{aligned}
$$

Table 2: Non-trivial radical $p$-subgroups of $\mathbb{B}$ with $p \geqslant 5$.

\begin{tabular}{lllrr}
\hline$R$ & $C(R)$ & $N$ & $\mathcal{W}_{N}$ & ${ }^{W_{\hat{N}}}$ \\
\hline 7 & $7 \times 2 \cdot L_{3}(4): 2$ & $\left(7: 3 \times 2 \cdot L_{3}(4): 2\right): 2$ & & \\
$7^{2}$ & $2^{2} \times 7^{2}$ & $\left(2^{2} \times 7^{2}:\left(3 \times 2 A_{4}\right)\right): 2$ & 24 & 24 \\
5 & $5 \times \mathrm{HS}: 2$ & $5: 4 \times \mathrm{HS}: 2$ & & \\
$5^{2}$ & $5^{2} \times S_{5}$ & $5^{2}: 4 S_{4} \times S_{5}$ & & \\
$5^{3}$ & $5^{3}$ & $5^{3} \cdot L_{3}(5)$ & & \\
$5_{+}^{1+4}$ & 5 & $5_{+}^{1+4} \cdot 2_{-}^{1+4} \cdot A_{5} \cdot 4$ & 30 & 12 \\
$5^{2} \cdot 5_{+}^{1+2}$ & $5^{2}$ & $5^{2} \cdot 5_{+}^{1+2} \cdot \mathrm{GL}_{2}(5)$ & 4 & 4 \\
$5_{+}^{1+4} \cdot 5$ & 5 & $5_{+}^{1+4} \cdot 5 \cdot 4^{2}$ & 16 & 16 \\
\hline
\end{tabular}


Since $\mathbb{B}$ has exactly two classes, $5 A$ and $5 B$, of elements of order 5 and $|N(5 A)|_{5}=5^{4}$, it follows that a generator of $C_{M_{2}}\left(5_{+}^{1+4}\right)$ is in a $5 B$-class, and so we may suppose that $N_{M_{2}}\left(5_{+}^{1+4}\right) \leqslant M_{3}=N(5 B)$. In particular, $N_{M_{2}}\left(5_{+}^{1+4}\right) \neq N_{\mathbb{B}}\left(5_{+}^{1+4}\right)$.

Since $\left|N_{M_{2}}\left(5^{2} .5^{1+2}\right)\right|_{5}=5^{6}$, it follows that $M_{1}$ and $M_{4}$ have no subgroup conjugate to $N_{M_{2}}\left(5^{2} \cdot 5^{1+2}\right)$. Applying Lemma 2.3 , we see that $N_{M_{2}}\left(5^{2} \cdot 5^{1+2}\right) \mathbb{B}_{\mathbb{B}} M_{3}$, so by Lemma 2.2 , $5^{2} \cdot 5^{1+2}$ is radical in $\mathbb{B}$ with $N_{\mathbb{B}}\left(5^{2} \cdot 5^{1+2}\right)=N_{M_{2}}\left(5^{2} \cdot 5^{1+2}\right)$. This classifies the radical 5subgroups of $\mathbb{B}$.

Case (3) Suppose that $p=3$. By [16, Theorem 5.7], $\mathbb{B}$ has seven maximal 3-local subgroups:

$$
\begin{aligned}
& M_{1}=N(3 B)=3_{+}^{1+8}: 2_{-}^{1+6} \cdot U_{4}(2): 2 ; \quad M_{2}=N\left(3 B^{2}\right)=3^{2} \cdot 3^{3} \cdot 3^{6} \cdot\left(S_{4} \times 2 S_{4}\right) ; \\
& M_{3}=N\left(3 B^{3}\right)=3^{3} \cdot 3^{6} \cdot\left(L_{3}(3) \times D_{8}\right) ; \quad M_{4}=N\left(3 B^{3}\right)=3^{3} \cdot 3 \cdot 3^{3} \cdot 3^{3} \cdot\left(L_{3}(3) \times 2\right) \text {; } \\
& M_{5}=N(3 A)=S_{3} \times \mathrm{Fi}_{22}: 2 ; \quad M_{6}=N\left(3 A^{2}\right)=\left(3^{2}: D_{8} \times U_{4}(3): 2^{2}\right) .2 ; \\
& M_{7}=N\left(3^{6}\right)=3^{6}:\left(2 \times L_{4}(3)\right): 2 \text {. }
\end{aligned}
$$

We first classify the radical subgroups of each $M_{i}$, applying the modified local strategy, and do the fusions in $\mathbb{B}$ using Lemmas 2.3 and 2.2.

Case (3.1) We may take

$$
\mathcal{R}_{0}\left(M_{1}, 3\right)=\left\{3^{1+8}, 3^{1+8} \cdot 3,3^{1+8} \cdot 3^{2}, 3^{1+8} \cdot 3^{3}, 3^{1+8} \cdot 3^{1+2}, S\right\} .
$$

Since $C_{M_{1}}(R)=3=Z(R)$ for each $R \in \mathcal{R}_{0}\left(M_{1}, 3\right)$, it follows that $N_{\mathbb{B}}(R) \leqslant \mathbb{B} M_{1}$, so $R$ is radical in $\mathbb{B}$ with $N_{M_{1}}(R)=N_{\mathbb{B}}(R)$. Thus we may suppose that $\mathcal{R}_{0}\left(M_{1}, 3\right) \subseteq \mathcal{R}_{0}(\mathbb{B}, 3)$.

Table 3: Non-trivial radical 3-subgroups of $\mathbb{B}$.

\begin{tabular}{lllrr}
\hline$R$ & $C(R)$ & $N(R)$ & $W_{N}$ & $W_{\hat{N}}$ \\
\hline 3 & $3 \times \mathrm{Fi}_{22}: 2$ & $S_{3} \times \mathrm{Fi}_{22}: 2$ & & \\
$3^{2}$ & $3^{2} \times U_{4}(3): 2^{2}$ & $\left(3^{2}: D_{8} \times U_{4}(3): 2^{2}\right) \cdot 2$ & & \\
$3^{6}$ & $3^{6}$ & $3^{6}:\left(2 \times L_{4}(3): 2\right): 2$ & 5 & 2 \\
$3_{+}^{1+8}$ & 3 & $3_{+}^{1+8} \cdot 2_{-}^{1+6} \cdot U_{4}(3) \cdot 2$ & 11 & 1 \\
$3^{3} \cdot 3^{6}$ & $3^{3}$ & $3^{3} \cdot 3^{6} \cdot\left(L_{3}(3) \times D_{8}\right)$ & 5 & 2 \\
$3_{+}^{1+8} \cdot 3$ & 3 & $3_{+}^{1+8} \cdot 3 \cdot 2^{2} \cdot 2^{4} \cdot 3^{2} \cdot D_{8}$ & 10 & 4 \\
$3^{3} \cdot 3 \cdot 3^{3} \cdot 3^{3}$ & $3^{3}$ & $3^{3} \cdot 3 \cdot 3^{3} \cdot 3^{3} \cdot\left(L_{3}(3) \times 2\right)$ & 2 & 2 \\
$3_{+}^{1+8} \cdot 3^{2}$ & 3 & $3_{+}^{1+8} \cdot 3^{2} \cdot\left(D_{8} \times 2 S_{4}\right)$ & 10 & 4 \\
$3^{2} \cdot 3^{3} \cdot 3^{6}$ & $3^{2}$ & $3^{2} \cdot 3^{3} \cdot 3^{6} \cdot\left(S_{4} \times 2 S_{4}\right)$ & 4 & 0 \\
$3_{+}^{1+8} \cdot 3^{3}$ & 3 & $3_{+}^{1+8} \cdot 3^{3} \cdot 2^{4} \cdot S_{3}$ & 8 & 0 \\
$3_{+}^{1+8} \cdot 3_{+}^{1+2}$ & 3 & $3_{+}^{1+8} \cdot 3_{+}^{1+2} \cdot\left(2 \times 2 S_{4}\right)$ & 4 & 4 \\
$3^{2} \cdot 3^{3} \cdot 3^{6} \cdot 3$ & $3^{2}$ & $3^{2} \cdot 3^{3} \cdot 3^{6} \cdot 3 \cdot\left(2 \times 2 S_{4}\right)$ & 4 & 4 \\
$S$ & 3 & $S \cdot 2^{3}$ & 8 & 8 \\
\hline
\end{tabular}


Case (3.2) Applying the local strategy, we find four classes of radical subgroups of $M_{2}$; one of them, $R$, has order $3^{12}$ satisfying $C_{M_{2}}(R)=Z(R)=3$ and $N_{M_{2}}(R)=R .2 .\left(S_{4} \times 2\right)$. Thus a generator of $Z(R)$ is a $3 B$-element as $|N(3 A)|_{3}=3^{10}$, and we may suppose that $N_{M_{2}}(R) \leqslant M_{1}$. By Lemma 2.3 and (5.3), $R$ is radical in $M_{1}$, and by the local structures, $R=M_{1} 3^{1+8} \cdot 3^{3}$.

Another radical subgroup $Q$ of $M_{2}$ also has order $3^{12}$ and $C_{M_{2}}(Q)=3^{2}=Z(Q)$. So $N_{\mathbb{B}}(Q) \leqslant \mathbb{B} M_{2}$ and $Q$ is radical in $\mathbb{B}$. We may take

$$
\mathcal{R}_{0}\left(M_{2}, 3\right)=\left\{3^{2} \cdot 3^{3} \cdot 3^{6}, 3^{1+8} \cdot 3^{3}, 3^{2} \cdot 3^{3} \cdot 3^{6} \cdot 3, S\right\},
$$

and $N_{\mathbb{B}}(R)=N_{M_{2}}(R)$ for each $R \in \mathcal{R}_{0}\left(M_{2}, 3\right)$, so we may suppose that $\mathcal{R}_{0}\left(M_{2}, 3\right) \subseteq$ $\mathcal{R}_{0}(\mathbb{B}, 3)$.

Case (3.3) $M_{3}$ has 4 classes of radical subgroups; one of them, $R$, has order $3^{11}$ with $C_{M_{3}}(R)=Z(R)=3^{2}$ and $N_{M_{3}}(R)=R \cdot\left(2 S_{4} \times D_{8}\right)$. Thus $Z(R)$ is $3 B$-pure, and by [16, Theorem 4.4] we may suppose that $N_{M_{3}}(R) \leqslant M_{2}$. By (5.4) and Lemma 2.2, $R$ is non-radical in $\mathbb{B}$.

Another radical subgroup $Q$ of $M_{3}$ also has order $3^{11}$ and $C_{M_{3}}(Q)=Z(Q)=3$, so that $Z(Q)$ is generated by a $3 B$-element and we may suppose that $N_{M_{3}}(Q) \leqslant M_{1}$. By the local structures, we can identify $Q$ with $3_{+}^{1+8} \cdot 3^{2}$. We may take

$$
\mathcal{R}_{0}\left(M_{3}, 3\right)=\left\{3^{3} \cdot 3^{6}, 3^{3} \cdot 3^{6} \cdot 3^{2}, 3_{+}^{1+8} \cdot 3^{2}, 3_{+}^{1+8} \cdot 3^{2} \cdot 3\right\}
$$

and $N_{\mathbb{B}}(R) \neq N_{M_{3}}(R)$ for $R \in \mathcal{R}_{0}\left(M_{2}, 3\right) \backslash\left\{3^{3} \cdot 3^{6}, 3_{+}^{1+8} \cdot 3^{2}\right\}$. Moreover, $C_{M_{3}}\left(3^{3} \cdot 3^{6} \cdot 3^{2}\right)=$ $3^{2}, C_{M_{3}}\left(3_{+}^{1+8} \cdot 3^{2} \cdot 3\right)=3$, and

$$
N_{M_{3}}(R)= \begin{cases}3^{3} \cdot 3^{6} \cdot 3^{2} \cdot\left(2 S_{4} \times D_{8}\right) & \text { if } R=3^{3} \cdot 3^{6} \cdot 3^{2} \\ 3_{+}^{1+8} \cdot 3^{2} \cdot 3 \cdot\left(2^{2} \times D_{8}\right) & \text { if } R=3_{+}^{1+8} \cdot 3^{2} \cdot 3\end{cases}
$$

Case (3.4) The fusions in $\mathbb{B}$ of the radical subgroups of $M_{4}$ with subgroups in other $\mathcal{R}_{0}\left(M_{i}, 3\right)$ can be done similarly to that of Case (3.2). We may take

$$
\mathcal{R}_{0}\left(M_{4}, 3\right)=\left\{3^{3} \cdot 3 \cdot 3^{3} \cdot 3^{3}, 3^{2} \cdot 3^{3} \cdot 3^{6} \cdot 3,3^{1+8} \cdot 3^{1+2}, S\right\}
$$

and $N_{\mathbb{B}}(R)=N_{M_{4}}(R)$ for each $R \in \mathcal{R}_{0}\left(M_{4}, 3\right)$, so we may suppose that $\mathcal{R}_{0}\left(M_{4}, 3\right) \subseteq$ $\mathcal{R}_{0}(\mathbb{B}, 3)$.

Case (3.5) $M_{7}$ has six classes of radical subgroups $R$, three of which have centralizer $C_{M_{7}}(R)=Z(R)=3$, and $\left|N_{M_{7}}(R)\right|_{3} \geqslant 3^{11}$. So a generator of $Z(R)$ is a $3 B$-element, and we may suppose that $N_{M_{7}}(R) \leqslant M_{1}$. By (5.3) and the local structures, we find that one of the subgroups $R$ is conjugate to $3^{1+8} .3$, and the other two are not radical in $\mathbb{B}$.

A radical subgroup $Q$ of $M_{7}$ has order $3^{9}$ and $C_{M_{7}}(Q)=Z(Q)=3^{3}$. Since we have $\left|C_{M_{7}}(x)\right|_{3} \geqslant 3^{11}$ for each $x \in Z(Q), Z(Q)$ is $3 B$-pure, and by [16, Theorem 4.4], $N_{M_{7}}(Q)=Q \cdot\left(2^{2} \times L_{3}(3)\right) \leqslant_{\mathbb{B}} M_{i}$ for some $i=1,2,3,4$. By Lemma 2.2 and equations (5.3)-(5.6), $Q$ is not a radical subgroup of $\mathbb{B}$.

Similarly, $M_{7}$ has a radical subgroup $W$ of order $3^{11}$ with $Z(W)=3^{2}$, so that $Z(W)$ is $3 B$-pure and $N_{M_{7}}(W)=W \cdot\left(2 \times 2 S_{4}: 2\right): 2 \leqslant \mathbb{B} M_{i}$ for some $i=1,2,3,4$. This implies that $W$ is not radical in $\mathbb{B}$. Thus we may take

$$
\mathcal{R}_{0}\left(M_{7}, 3\right)=\left\{3^{6}, 3^{3+6}, 3_{+}^{1+8} \cdot 3,3^{6} \cdot 3^{2+3}, 3^{2} \cdot 3^{3} \cdot 3^{6}, 3^{3+6} \cdot 3^{1+2}\right\},
$$

and $N_{M_{7}}(R) \neq N_{\mathbb{B}}(R)$ for $R \in \mathcal{R}_{0}\left(M_{7}, 3\right) \backslash\left\{3^{6}, 3_{+}^{1+8} .3\right\}$. 
Moreover,

$$
\begin{aligned}
& C_{M_{7}}\left(3^{3+6}\right)=3^{3}, \\
& C_{M_{7}}\left(3_{+}^{1+8} \cdot 3\right) \cong C_{M_{7}}\left(3^{6} \cdot 3^{2+3}\right) \cong C_{M_{7}}\left(3^{3+6} \cdot 3^{1+2}\right)=3, \\
& C_{M_{7}}\left(3^{2} \cdot 3^{3} \cdot 3^{6}\right)=3^{2},
\end{aligned}
$$

and

$$
N_{M_{7}}(R)= \begin{cases}3^{3+6} \cdot\left(2^{2} \times L_{3}(3)\right) & \text { if } R=3^{3+6} \\ 3^{6} \cdot 3^{2+3} \cdot\left(2^{2} \times 2 S_{4}\right) & \text { if } R=3^{6} \cdot 3^{2+3} \\ 3^{2} \cdot 3^{3} \cdot 3^{6} \cdot\left(2 \times 2 S_{4}: 2\right) \cdot 2 & \text { if } R=3^{2} \cdot 3^{3} \cdot 3^{6} \\ 3^{3+6} \cdot 3^{1+2} \cdot 2^{3} \cdot 2^{2} & \text { if } R=3^{3+6} \cdot 3^{1+2}\end{cases}
$$

Case (3.6) Since $M_{5}=S_{3} \times \mathrm{Fi}_{22}: 2$, it follows that each radical subgroup $R$ is of the form $3 \times R_{1}$ for some $R_{1} \in \mathcal{R}_{0}\left(\mathrm{Fi}_{22}: 2,3\right)$. The fusion in $\mathbb{B}$ of elements of order 3 in $\mathrm{Fi}_{22}$ is given by [16, Proposition 3.1]. The radical subgroups of $M_{5}$ and their local structures are given in Table 4.

Note that $R=3^{2}$ is $3 A$-pure, so that $N_{\mathbb{B}}\left(3^{2}\right) \neq N_{M_{5}}\left(3^{2}\right)$. By [16, Proposition 3.2], $\mathbb{B}$ has a unique class of elementary abelian groups of order $3^{6}$ containing $3 A$-elements, and so $M_{7}=\mathbb{B} N_{\mathbb{B}}\left(3^{6}\right) \neq N_{M_{5}}\left(3^{6}\right)$.

Since the commutator subgroup $\left[3 \times 3_{+}^{1+6}, 3 \times 3_{+}^{1+6}\right]=\left(3 \times 3_{+}^{1+6}\right)^{\prime}=3$ is $3 B$-pure, $N_{\mathbb{B}}\left(3 \times 3_{+}^{1+6}\right) \leqslant \mathbb{B} N(3 B)=M_{1}$. But $N_{M_{5}}(R) \leqslant M_{5} N_{M_{5}}\left(3 \times 3_{+}^{1+6}\right)$ for $R=3 \times 3_{+}^{1+6} .3$ and $R=3 \times 3^{5} .3^{3}$; also, $C_{M_{5}}(R) \cong 3^{2}=C_{M_{5}}\left(3 \times 3_{+}^{1+6}\right)$, so $N_{\mathbb{B}}(R) \leqslant \mathbb{B} M_{1}$, and by Lemma 2.2 and (5.3), none of them is radical in $\mathbb{B}$.

Suppose that $R=3 \times 3^{5}: 3_{+}^{1+2}$. Then $\left[R, R^{\prime}\right]=Z\left(3^{5}: 3_{+}^{1+2}\right)=3^{2}$ is $3 B$-pure, $N_{\mathbb{B}}(R) \leqslant \mathbb{B}$ $N\left(3 B^{2}\right)=M_{2}$ and, by (5.4), $R$ is non-radical in $\mathbb{B}$.

Since $\left(3 \times 3^{3+3}\right)^{\prime}=3^{3}$ is $3 B$-pure, $N_{\mathbb{B}}(R) \leqslant \mathbb{B} M_{4}$ and, by (5.6), $R$ is non-radical in $\mathbb{B}$.

It follows that $N_{M_{5}}(R) \neq N_{\mathbb{B}}(R)$ for any $R \in \mathcal{R}_{0}\left(M_{5}, 3\right) \backslash\{3\}$.

Table 4: Radical 3-subgroups of $S_{3} \times \mathrm{Fi}_{22}: 2$.

\begin{tabular}{lll}
\hline$R$ & $C(R)$ & $N_{M_{5}}(R)$ \\
\hline 3 & $3 \times \mathrm{Fi}_{22}: 2$ & $S_{3} \times \mathrm{Fi}_{22}: 2$ \\
$3^{2}$ & $3^{2} \times U_{4}(3): 2^{2}$ & $S_{3} \times\left(S_{3} \times U_{4}(3): 2\right) \cdot 2$ \\
$3^{6}$ & $3^{6}$ & $S_{3} \times 3^{5}:\left(2 \times U_{4}(2): 2\right)$ \\
$3 \times 3^{3+3}$ & $3^{4}$ & $S_{3} \times 3^{3+3}: L_{3}(3)$ \\
$3 \times 3_{+}^{1+6}$ & $3^{2}$ & $S_{3} \times 3_{+}^{1+6} \cdot 2^{3+4}: 3^{2} \cdot 2^{2}$ \\
$3 \times 3_{+}^{1+6} .3$ & $3^{2}$ & $S_{3} \times 3_{+}^{1+6} \cdot 3: 2 S_{4}$ \\
$3 \times 3^{5} .3^{3}$ & $3^{2}$ & $S_{3} \times 3^{5} \cdot 3^{3} \cdot\left(2 \times S_{4}\right) \cdot 2$ \\
$3 \times 3^{5} .3^{1+2}$ & $3^{3}$ & $S_{3} \times 3^{5} \cdot 3^{1+2} \cdot 2 S_{4} \cdot 2$ \\
$3 \times 3_{+}^{1+6} .3^{2}$ & $3^{2}$ & $S_{3} \times 3^{1+6} \cdot 3^{2} \cdot 2^{3}$ \\
\hline
\end{tabular}


Case (3.7) We may take

$$
\mathcal{R}_{0}\left(M_{6}, 3\right)=\left\{3^{2}, 3^{6}, 3^{2} \times 3^{1+4}, 3^{3} \cdot 3^{2} \cdot 3^{3}\right\}
$$

in addition, $C_{M_{6}}\left(3^{2} \times 3^{1+4}\right)=3^{3}=C_{M_{6}}\left(3^{3} \cdot 3^{2} \cdot 3^{3}\right)$ and

$$
N_{M_{6}}(R)= \begin{cases}\left(3^{2}: D_{8} \times 3^{4}: A_{6}: 2^{2}\right) \cdot 2 & \text { if } R=3^{6} \\ \left(3^{2}: D_{8} \times 3^{1+4} \cdot 2 S_{4}: 2^{2}\right) \cdot 2 & \text { if } R=3^{2} \times 3^{1+4} \\ 3^{3} \cdot 3^{2} \cdot 3^{3} \cdot 2^{3} \cdot 2^{2} \cdot 2^{3} & \text { if } R=3^{3} \cdot 3^{2} \cdot 3^{3}\end{cases}
$$

Thus $N_{M_{6}}\left(3^{6}\right) \neq{ }_{\mathbb{B}} M_{7}$.

Since $C_{M_{6}}\left(3^{2}\right)=\mathbb{B} C_{M_{5}}\left(3^{2}\right)$, we may suppose that $R=3^{2} \times 3^{1+4} \leqslant M_{5}$. Now $R^{\prime}=3$ is $3 B$-pure, and so $N_{\mathbb{B}}(R) \leqslant \mathbb{B} N(3 B)$ and $R$ is not radical in $\mathbb{B}$.

It follows that $N_{M_{6}}(R) \neq N_{\mathbb{B}}(R)$ for each $R \in \mathcal{R}_{0}\left(M_{6}, 3\right) \backslash\left\{3^{2}\right\}$.

Thus the radical 3-subgroups of $\mathbb{B}$ are listed in Table 3 , and the centralizers and normalizers are given by MAGMA.

Lemma 5.2. Let $G=\mathbb{B}$ and $B_{0}=B_{0}(G)$, let $\mathrm{Blk}^{+}(G, p)$ be the set of $p$-blocks with a non-trivial defect group, and let $\operatorname{Irr}^{+}(G)$ be the characters of $\operatorname{Irr}(G)$ with positive p-defect. If a defect group $D(B)$ of $B$ is cyclic, then $\operatorname{Irr}(B)$ is given by [11, p. 387].

(a) If $p=7$, then $\operatorname{Blk}^{+}(G, p)=\left\{B_{i} \mid 0 \leqslant i \leqslant 8\right\}$ such that $D\left(B_{i}\right) \cong 7^{2}$ when $0 \leqslant i \leqslant 2$ while $D\left(B_{j}\right) \cong 7$ when $3 \leqslant j \leqslant 8$. In the notation of [7, p. 208],

$$
\begin{aligned}
\operatorname{Irr}\left(B_{1}\right)= & \left\{\chi_{2}, \chi_{4}, \chi_{10}, \chi_{17}, \chi_{20}, \chi_{21}, \chi_{22}, \chi_{27}, \chi_{42}, \chi_{45}, \chi_{46}, \chi_{55}, \chi_{63}, \chi_{73},\right. \\
& \left.\chi_{77}, \chi_{85}, \chi_{88}, \chi_{100}, \chi_{103}, \chi_{106}, \chi_{118}, \chi_{121}, \chi_{134}, \chi_{161}, \chi_{166}, \chi_{167}, \chi_{176}\right\}, \\
\operatorname{Irr}\left(B_{2}\right)= & \left\{\chi_{7}, \chi_{9}, \chi_{11}, \chi_{12}, \chi_{31}, \chi_{32}, \chi_{33}, \chi_{38}, \chi_{43}, \chi_{64}, \chi_{67}, \chi_{69}, \chi_{74}, \chi_{76},\right. \\
& \left.\chi_{90}, \chi_{91}, \chi_{92}, \chi_{94}, \chi_{96}, \chi_{105}, \chi_{107}, \chi_{112}, \chi_{115}, \chi_{117}, \chi_{119}, \chi_{126}, \chi_{135}\right\},
\end{aligned}
$$

and

$$
\operatorname{Irr}\left(B_{0}\right)=\operatorname{Irr}^{+}(G) \backslash\left(\bigcup_{i=1}^{8} \operatorname{Irr}\left(B_{i}\right)\right)
$$

Moreover, $\ell\left(B_{0}\right)=\ell\left(B_{1}\right)=24, \ell\left(B_{2}\right)=21, \ell\left(B_{i}\right)=6$ for $3 \leqslant i \leqslant 7$ and $\ell\left(B_{8}\right)=3$.

(b) If $p=5$, then $\mathrm{Blk}^{+}(G, p)=\left\{B_{i} \mid 0 \leqslant i \leqslant 6\right\}$ such that $D\left(B_{i}\right) \cong 5^{2}$ for $i=1,2$, and $D\left(B_{j}\right) \cong 5$ for $3 \leqslant j \leqslant 6$. In the notation of [7, p. 208],

$$
\begin{aligned}
\operatorname{Irr}\left(B_{1}\right)= & \left\{\chi_{5}, \chi_{9}, \chi_{14}, \chi_{18}, \chi_{32}, \chi_{40}, \chi_{54}, \chi_{56}, \chi_{81}, \chi_{93},\right. \\
& \left.\chi_{97}, \chi_{105}, \chi_{115}, \chi_{130}, \chi_{135}, \chi_{144}, \chi_{151}, \chi_{153}, \chi_{159}, \chi_{177}\right\}, \\
\operatorname{Irr}\left(B_{2}\right)= & \left\{\chi_{27}, \chi_{29}, \chi_{34}, \chi_{44}, \chi_{55}, \chi_{64}, \chi_{67}, \chi_{74}, \chi_{80}, \chi_{82},\right. \\
& \left.\chi_{85}, \chi_{91}, \chi_{92}, \chi_{101}, \chi_{109}, \chi_{134}, \chi_{136}, \chi_{156}, \chi_{162}, \chi_{176}\right\},
\end{aligned}
$$

and

$$
\operatorname{Irr}\left(B_{0}\right)=\operatorname{Irr}^{+}(G) \backslash\left(\bigcup_{i=1}^{6} \operatorname{Irr}\left(B_{i}\right)\right)
$$

Moreover, $\ell\left(B_{0}\right)=51, \ell\left(B_{1}\right)=\ell\left(B_{2}\right)=16$, and $\ell\left(B_{j}\right)=4$ for $3 \leqslant j \leqslant 6$. 
(c) If $p=3$, then $\mathrm{Blk}^{+}(G, p)=\left\{B_{i} \mid 0 \leqslant i \leqslant 5\right\}$ such that $D\left(B_{i}\right) \cong 3^{2}$ for $i=1,2,3$, and $D\left(B_{j}\right) \cong 3$ for $4 \leqslant j \leqslant 5$. In the notation of [7, p. 208],

$$
\operatorname{Irr}\left(B_{i}\right)= \begin{cases}\left\{\chi_{30}, \chi_{33}, \chi_{79}, \chi_{97}, \chi_{107}, \chi_{123}, \chi_{126}, \chi_{151}, \chi_{164}\right\} & \text { if } i=1 \\ \left\{\chi_{43}, \chi_{64}, \chi_{96}, \chi_{109}, \chi_{111}, \chi_{150}, \chi_{171}, \chi_{173}, \chi_{181}\right\} & \text { if } i=2, \\ \left\{\chi_{63}, \chi_{76}, \chi_{91}, \chi_{100}, \chi_{105}, \chi_{106}, \chi_{154}, \chi_{156}, \chi_{162}\right\} & \text { if } i=3\end{cases}
$$

Moreover, $\ell\left(B_{0}\right)=71, \ell\left(B_{1}\right)=\ell\left(B_{2}\right)=7, \ell\left(B_{3}\right)=5$ and $\ell\left(B_{j}\right)=2$ for $j=4,5$.

In addition, let $\hat{G}=2 \cdot \mathbb{B}$ and let $\xi$ be the faithful linear character of $\operatorname{Irr}(Z(\hat{G}))$. If $p \in\{3,5,7\}$, then there is a unique $p$-block $\hat{B}$ of $\hat{G}$ such that $\hat{B}$ covers the block $B(\xi)$ and the defect group $D(\hat{B})$ is non-cyclic. In the notation of [7, p. 218], let

$\Omega= \begin{cases}\left\{\chi_{j}: j \in\{189,192,193,197,202,205,206,207,208,222,225,234\}\right\} & \text { if } p=7, \\ \left\{\chi_{j}: j \in\{204,205,206,209\}\right\} & \text { if } p=5, \\ \varnothing & \text { if } p=3 .\end{cases}$

Then

$$
\operatorname{Irr}(\hat{B})=\operatorname{Irr}^{+}(\hat{G}) \backslash\left(\Omega \cup \operatorname{Irr}^{+}(G)\right)
$$

and $\ell(\hat{B})$ is 24,33 or 31 when $p$ is 7,5 or 3 .

Proof. If $B \in \operatorname{Blk}(G, p)$ is non-principal with $D=D(B)$, then $\operatorname{Irr}^{0}(C(D) D / D)$ has a non-trivial character $\theta$ and $N(\theta) / C(D) D$ is a $p^{\prime}$-group, where $N(\theta)$ is the stabilizer of $\theta$ in $N(D)$. By [11, p. 387], we may suppose that $D$ is non-cyclic, so that by Proposition 5.1, $D=p^{2}$.

If $p=7$, then $C(D)=2^{2} \times 7^{2}$ and $N(D)=\left(2^{2} \times 7^{2}:\left(3 \times 2 A_{4}\right)\right): 2$, so that $N(D)$ has 3 orbits on $\operatorname{Irr}^{0}(C(D) D / D)$ and $\mathbb{B}$ has three blocks with defect group $D=7^{2}$.

If $p=5^{2}$, then $C(D)=5^{2} \times S_{5}$ and $N(D)=5^{2}: 4 S_{4} \times S_{5}$, so that $N(D)$ has two orbits on $\operatorname{Irr}^{0}(C(D) D / D)$ and $\mathbb{B}$ has two blocks with defect group $D=5^{2}$.

If $p=3^{2}$, then $C(D)=3^{2} \times U_{4}(3): 2^{2}$ and $N(D)=\left(3^{2}: D_{8} \times U_{4}(3): 2^{2}\right) .2$, so that $N(D)$ has three orbits on $\operatorname{Irr}^{0}(C(D) D / D)$ and $\mathbb{B}$ has three blocks with defect group $D=3^{2}$.

Using the method of central characters, $\operatorname{Irr}(B)$ is as above. If $D(B)$ is cyclic, then $\ell(B)$ is given by [11, p. 387].

If $p=7$ and $B \in\left\{B_{1}, B_{2}\right\}$, then the non-trivial elements of $D(B)$ are conjugate in $\mathbb{B}$, and $C(x)=7 \times 2 \cdot L_{3}(4): 2$ for any $1 \neq x \in D(B)$. It follows that

$$
\mathrm{k}(B)=\ell(B)+\sum_{b \in \operatorname{Blk}(C(x), B)} \ell(b),
$$

where $\operatorname{Blk}(C(x), B)=\left\{b \in \operatorname{Blk}(C(x)): b^{G}=B\right\}$. In particular, for $b \in \operatorname{Blk}(C(x), B)$, $b=B_{0}(7) \times b^{\prime}$ for some block $b^{\prime} \in \operatorname{Blk}\left(2 \cdot L_{3}(4): 2\right)$ with cyclic defect group 7. Now $C_{2} \cdot L_{3}(4): 2(7)=7 \times 2^{2}, N_{2} \cdot L_{3}(4): 2(7)=2^{2} \times 7: 3$, and so $\ell(b)=\ell\left(b^{\prime}\right)=\mathcal{W}\left(b^{\prime}\right)=3$. Thus if an outer involution in $\left(2^{2} \times 7^{2}:\left(3 \times 2 A_{4}\right)\right): 2$ stabilizes a root block of $B$, then $\ell(B)=27-3=24$; otherwise, $\ell(B)=27-6=21$. We may suppose that $\ell\left(B_{1}\right)=24$ and $\ell\left(B_{2}\right)=21$.

If $p=5$ and $B$ equals $B_{1}$ or $B_{2}$, then the non-trivial elements of $D(B)$ are of type $5 A$, and $C(5 A)=5 \times$ HS:2, so that (5.11) holds with $C(x)=5 \times$ HS:2 and $b=B_{0}(5) \times b^{\prime}$, where $b^{\prime} \in \operatorname{Blk}(\mathrm{HS}: 2)$ with cyclic defect group 5 . Thus $\mathcal{W}\left(b^{\prime}\right)=\ell\left(b^{\prime}\right)=4$ as $N_{\mathrm{HS}: 2}(5)=5: 4 \times S_{5}$, and $\ell(B)=20-4=16$. 
If $p=3$ and $B \in\left\{B_{1}, B_{2}, B_{3}\right\}$, then the non-trivial elements of $D(B)$ are of type $3 A$, and $C(3 A)=S_{3} \times \mathrm{Fi}_{22}: 2$, so that $b=B_{0}\left(S_{3}\right) \times b^{\prime}$ for some $b^{\prime} \in \mathrm{Blk}\left(\mathrm{Fi}_{22}: 2\right)$ with cyclic defect group 3, where $b \in \operatorname{Blk}\left(S_{3} \times \mathrm{Fi}_{22}: 2, B\right)$. Now

$$
\begin{aligned}
& C_{\mathrm{Fi}_{22}: 2}(3)=3 \times U_{4}(3): 2^{2} ; \\
& N_{\mathrm{Fi}_{22}: 2}(3)=\left(S_{3} \times U_{4}(3): 2\right) .2 ; \quad N_{\mathrm{Fi}_{22}: 2}(3) /\left(3 \times U_{4}(3)\right)=2^{3} .
\end{aligned}
$$

Thus, if an element of $N_{\mathrm{Fi}_{22}: 2}(3) \backslash C_{\mathrm{Fi}_{22}: 2}(3)$ stabilizes the root block of $b^{\prime}$, then $\mathcal{W}\left(b^{\prime}\right)=$ $\ell\left(b^{\prime}\right)=2$; otherwise, $\mathcal{W}\left(b^{\prime}\right)=\ell\left(b^{\prime}\right)=4$. We may suppose that $\ell\left(B_{i}\right)=k\left(B_{i}\right)-2=$ $9-2=7$ when $i=1,2$ and $\ell\left(B_{3}\right)=k\left(B_{3}\right)-4=9-4=5$.

If $\ell_{p}(G)$ is the number of $p$-regular $G$-conjugacy classes, then $\ell_{7}(G)=165, \ell_{5}(G)=$ 144 and $\ell_{3}(G)=103$. Thus $\ell\left(B_{0}\right)$ can be calculated by the following equation, due to Brauer:

$$
\ell_{p}(G)=\sum_{B \in \mathrm{Blk}^{+}(G, p)} \ell(B)+\left|\operatorname{Irr}^{0}(G)\right|,
$$

where $\left|\operatorname{Irr}^{0}(G)\right|$ is 63,45 or 9 when $p$ equals 7,5 or 3 .

The set $\operatorname{Irr}(\hat{B})$ is also determined using the method of central characters, and $\ell(\hat{B})$ is given by (5.12) with $G$ replaced by $\hat{G}$, where $\ell_{7}(\hat{G})=220, \ell_{5}(\hat{G})=189$ and $\ell_{3}(\hat{G})=139$.

TheOREM 5.3. Let $G=2 . \mathbb{B}$, and let $B$ be a p-block of $G$ with a non-cyclic defect group. If $p \geqslant 3$, then the number of $B$-weights is the number of irreducible Brauer characters of $B$.

Proof. We may suppose that $p$ is 3,5 or 7. If $B=B_{0}$ or $\hat{B}$, then

$$
\mathcal{W}(B)=\sum_{R} \mathcal{W}_{H},
$$

where $R \in \mathcal{R}_{0}(\mathbb{B}, p)$ with $\mathrm{d}\left(C_{\mathbb{B}}(R) R / R\right)=0$, and $H=N=N_{\mathbb{B}}(R)$ or $\hat{N}=N_{2 . \mathbb{B}}(R)$, according to whether $B$ equals $B_{0}$ or $\hat{B}$. Thus Theorem 5.3 follows by Lemma 5.2 and Tables 2 and 3.

Suppose that $p=7$. Then $\left|\operatorname{Irr}\left(N_{\mathbb{B}}\left(7^{2}\right) / 7^{2}\right)\right|=69, \mathbb{B}$ has sixty-nine weights of the form $\left(7^{2}, \varphi\right)$, twenty-four of which are $B_{0}$-weights. If $b \in \operatorname{Blk}\left(2^{2} \times 7^{2}\right)$ is stabilized by an outer involution of $\left(2^{2} \times 7^{2}:\left(3 \times 2 A_{4}\right)\right): 2$, then its canonical character $\theta$ is stabilized by $N_{\mathbb{B}}\left(7^{2}\right)$, so $\theta$ has an extension to $N_{\mathbb{B}}\left(7^{2}\right)$ and $b^{N_{\mathbb{B}}\left(7^{2}\right)}$ has twenty-four weight characters, which form all twenty-four $B_{1}$-weights. Finally, $B_{2}$ has $21=69-24-24$ weights.

If $p=5$ and $B$ is $B_{1}$ or $B_{2}$, then $N_{\mathbb{B}}(D) / C_{\mathbb{B}}(D) D=4 S_{4}$, which has sixteen irreducible characters, so that $B$ has sixteen weights of the form $(D, \varphi)$.

If $p=3$ and $B \in\left\{B_{1}, B_{2}\right\}$, then $N_{\mathbb{B}}(D) / C_{\mathbb{B}}(D) D$ is a semidihedral group $S D_{16}$, so that $B$ has seven weights of the form $(D, \varphi)$.

If $p=3$ and $B=B_{3}$, then $B$ has five weights of the form $(D, \varphi)$, since $N_{\mathbb{B}}(D) / D$ has nineteen irreducible characters of defect 0 , and fourteen of these are weight characters of $B_{1}$ or $B_{2}$.

\section{Radical chains}

Let $G=\mathbb{B}, C \in \mathcal{R}(G)$ and $N(C)=N_{G}(C)$. We will do some cancellations in the alternating sum of Uno's conjecture. We first list some radical $p$-chains $C(i)$ and their normalizers for certain integers $i$, and then reduce the proof of the conjecture to the subfamily $\mathcal{R}^{0}=\mathcal{R}^{0}(G)$ of $\mathcal{R}(G)$, where $\mathcal{R}^{0}(G)$ is the union of $G$-orbits of all $C(i)$. The subgroups of the $p$-chains in Tables 5 and 6 are given either by Tables 2 and 3, or in the proofs of Proposition 5.1 and Lemma 6.1. 
Lemma 6.1. Let $\mathcal{R}^{0}(G)$ be the $G$-invariant subfamily of $\mathcal{R}(G)$ such that

Then

$$
\mathcal{R}^{0}(G) / G= \begin{cases}\{C(i): 1 \leqslant i \leqslant 10\} & \text { with } C(i) \text { given in Table } 5 \text { if } p=5, \\ \{C(i): 1 \leqslant i \leqslant 36\} & \text { with } C(i) \text { given in Table } 6 \text { if } p=3 .\end{cases}
$$

$$
\sum_{C \in \mathcal{R}(G) / G}(-1)^{|C|} \mathrm{k}(N(C), B, d,[r])=\sum_{C \in \mathcal{R}^{0}(G) / G}(-1)^{|C|} \mathrm{k}(N(C), B, d,[r]) .
$$

Proof. Let $C \in \mathcal{R}(G)$ be given by (2.1), so that we may suppose that $P_{1} \in \mathcal{R}_{0}(G, p)$.

Case (1) Suppose that $p=5$. If $P_{1}=O_{5}\left(M_{1}\right)=5$, then $C \in_{G}\{C(2), C(3), C(4), C(5)\}$, since $\mathcal{R}_{0}\left(N_{\mathbb{B}}\left(5^{2}\right), 5\right)=\left\{5^{2}, 5^{3}\right\}$.

Let $\sigma: 1<Q=5^{3}<5^{2} .5^{1+2}$, so that $\sigma^{\prime}: 1<5^{2} .5^{1+2}$. Thus $\sigma$ and $\sigma^{\prime}$ satisfy the conditions of Lemma 2.1, so there is a bijection $g$ from $\mathcal{R}^{-}\left(\sigma, 5^{3}\right)$ onto $\mathcal{R}^{0}\left(\sigma, 5^{3}\right)$ such that $N\left(C^{\prime}\right)=N\left(g\left(C^{\prime}\right)\right)$ and $\left|C^{\prime}\right|=\left|g\left(C^{\prime}\right)\right|-1$ for each $C^{\prime} \in \mathcal{R}^{-}\left(\sigma, 5^{3}\right)$. So

$$
\mathrm{k}\left(N\left(C^{\prime}\right), B, d,[r]\right)=\mathrm{k}\left(N\left(g\left(C^{\prime}\right)\right), B, d,[r]\right),
$$

and we may suppose that $C \notin\left(\mathcal{R}^{-}\left(\sigma, 5^{3}\right) \cup \mathcal{R}^{0}\left(\sigma, 5^{3}\right)\right)$. In particular, we may suppose that $P_{1} \neq \neq_{G} 5^{2} .5^{1+2}$, and if $P_{1}=5^{3}$, then $P_{2} \neq \neq_{G} 5^{2} \cdot 5^{1+2}$. Similarly, let $C^{\prime}: 1<5^{3}<5^{1+4}<$ $5^{1+4} .5$ and $g\left(C^{\prime}\right): 1<5^{3}<5^{1+4} .5$. Then $N\left(C^{\prime}\right)=N\left(g\left(C^{\prime}\right)\right)$ and (6.2) holds; hence, if $P_{1}=5^{3}=O_{5}\left(M_{2}\right)$, we may suppose that $C \in_{G}\{C(6), C(7)\}$.

Let $C^{\prime}: 1<5^{1+4}<5^{1+4} .5$ and $g\left(C^{\prime}\right): 1<5^{1+4} .5$, so that $N\left(C^{\prime}\right)=N\left(g\left(C^{\prime}\right)\right)=$ $5^{1+4} .5 .4^{2}$ and (6.2) holds. If $P_{1}=5^{1+4}$, then we may suppose that $C={ }_{G} C(8)$. If $P_{1}=5^{2}$, then $C={ }_{G} C(9)$ or $C(10)$.

Case (2) Suppose that $p=3$.

Case (2.1) Let $R \in \mathcal{R}_{0}\left(M_{1}, 3\right) \backslash\left\{3^{1+8}\right\}$ and let $\sigma(R): 1<Q=3^{1+8}<R$, so that $\sigma(R)^{\prime}: 1<R$, where $\mathcal{R}_{0}\left(M_{1}, 3\right)$ is given by (5.3). Then $\sigma(R)$ and $\sigma(R)^{\prime}$ satisfy the conditions of Lemma 2.1 .

Table 5: Some radical 5-chains of $\mathbb{B}$.

\begin{tabular}{lll}
\hline \multicolumn{1}{c}{$C$} & \multicolumn{1}{c}{$N(C)$} \\
\hline$C(1)$ & 1 & $\mathbb{B}$ \\
$C(2)$ & $1<5$ & $5: 4 \times \mathrm{HS}: 2$ \\
$C(3)$ & $1<5<5^{2}$ & $5: 4 \times 5: 4 \times S_{5}$ \\
$C(4)$ & $1<5<5^{2}<5^{3}$ & $5: 4 \times 5: 4 \times 5: 4$ \\
$C(5)$ & $1<5<5 \times 5_{+}^{1+2}$ & $5: 4 \times 5_{+}^{1+2} .4 . D_{8}$ \\
$C(6)$ & $1<5^{3}$ & $5^{3} . L_{3}(5)$ \\
$C(7)$ & $1<5^{3}<5_{+}^{1+4}$ & $5_{+}^{1+4} \cdot \mathrm{GL}_{2}(5)$ \\
$C(8)$ & $1<5_{+}^{1+4}$ & $5_{+}^{1+4} .2_{-}^{1+4} . A_{5} \cdot 4$ \\
$C(9)$ & $1<5^{2}<5^{3}$ & $5^{2}: 4 S_{4} \times 5: 4$ \\
$C(10)$ & $1<5^{2}$ & $5^{2}: 4 S_{4} \times S_{5}$ \\
\hline
\end{tabular}


A similar proof to that of Case (1) shows that we may suppose that

$$
C \notin \bigcup_{R \in \mathcal{R}_{0}\left(M_{1}, 3\right) \backslash\left\{3^{1+8}\right\}}\left(\mathcal{R}^{-}\left(\sigma(R), 3^{1+8}\right) \cup \mathcal{R}^{0}\left(\sigma(R), 3^{1+8}\right)\right) .
$$

In particular, $P_{1} \notin_{G} \mathcal{R}_{0}\left(M_{1}, 3\right) \backslash\left\{3^{1+8}\right\}$, and if $P_{1}=3^{1+8}$, then $C=C(2)$.

We may suppose that

$$
P_{1} \in_{G}\left\{3,3^{2}, 3^{6}, 3^{3} \cdot 3^{6}, 3^{3} \cdot 3 \cdot 3^{3} \cdot 3^{3}, 3^{2} \cdot 3^{3} \cdot 3^{6}, 3^{2} \cdot 3^{3} \cdot 3^{6} \cdot 3\right\}
$$

Case (2.2) Let $\sigma: 1<Q=3^{2} \cdot 3^{3} \cdot 3^{6}<3^{2} \cdot 3^{3} \cdot 3^{6} .3$, so that $\sigma^{\prime}: 1<3^{2} \cdot 3^{3} \cdot 3^{6} \cdot 3$, where $3^{2} \cdot 3^{3} \cdot 3^{6}, 3^{2} \cdot 3^{3} \cdot 3^{6} \cdot 3 \in \mathcal{R}_{0}\left(M_{2}, 3\right)$ given by (5.4). Then $\sigma$ and $\sigma^{\prime}$ satisfy the conditions of Lemma 2.1. A similar proof to that of Case (1) shows that we may suppose that

$$
C \notin\left(\mathcal{R}^{-}\left(\sigma, 3^{2} \cdot 2^{3} \cdot 3^{6}\right) \cup \mathcal{R}^{0}\left(\sigma, 3^{2} \cdot 2^{3} \cdot 3^{6}\right)\right) .
$$

In particular, $P_{1} \neq_{G} 3^{2} \cdot 3^{3} \cdot 3^{6} \cdot 3$ and if $P_{1}=3^{2} \cdot 2^{3} \cdot 3^{6}$, then $P_{2} \neq_{G} 3^{2} \cdot 3^{3} \cdot 3^{6} .3$.

Let $C^{\prime}: 1<3^{2} \cdot 2^{3} \cdot 3^{6}<S$ and $g\left(C^{\prime}\right): 1<3^{2} \cdot 2^{3} \cdot 3^{6}<3^{1+8} \cdot 3^{3}<S$. Then $N\left(C^{\prime}\right)=$ $N\left(g\left(C^{\prime}\right)\right)=S .2^{3}$, and so (6.2) holds. Thus if $P_{1}=3^{2} \cdot 3^{3} \cdot 3^{6}=O_{3}\left(M_{2}\right)$, we may suppose that $C \in_{G}\{C(3), C(4)\}$.

Case (2.3) Let $C^{\prime}: 1<3^{3} .3^{6}<3^{1+8} .3^{2} .3$ and $g\left(C^{\prime}\right): 1<3^{3} .3^{6}<3^{1+8} .3^{2}<$ $3^{1+8} .3^{2} .3$, where $3^{1+8} .3^{2}, 3^{1+8} .3^{2} .3 \in \mathcal{R}_{0}\left(M_{3}, 3\right)$. Then $N\left(C^{\prime}\right)=N\left(g\left(C^{\prime}\right)\right)$, and $(6.2)$ holds. We may suppose that $C \neq_{G} C^{\prime}$ and $C \neq_{G} g\left(C^{\prime}\right)$, so that if $P_{1}=3^{3} .3^{6}$, we may suppose that $C \in_{G}\{C(5), C(6), C(7), C(8)\}$.

Case (2.4) Let $C^{\prime}: 1<3^{3} \cdot 3 \cdot 3^{3} \cdot 3^{3}<S$ and $g\left(C^{\prime}\right): 1<3^{3} \cdot 3 \cdot 3^{3} \cdot 3^{3}<3^{1+8} \cdot 3^{1+2}<S$, where $3^{3} \cdot 3.3^{3} \cdot 3^{3}, 3^{1+8} \cdot 3^{1+2} \in \mathcal{R}_{0}\left(M_{4}, 3\right)$. Then $N\left(C^{\prime}\right)=N\left(g\left(C^{\prime}\right)\right)=S .2^{3}$, and $(6.2)$ holds. We may suppose that $C \neq_{G} C^{\prime}$ and $C \neq_{G} g\left(C^{\prime}\right)$, so that if $P_{1}=3^{3} \cdot 3 \cdot 3^{3} \cdot 3^{3}$, we may suppose that $C \in_{G}\{C(9), C(10), C(11), C(12)\}$.

Case (2.5) Let $L=N_{M_{5}}\left(3^{6}\right)=S_{3} \times 3^{5}:\left(2 \times U_{4}(2): 2\right)$. We may take

$$
\mathcal{R}_{0}(L, 3)=\left\{3^{6}, 3 \times 3^{5} \cdot 3^{3}, 3 \times 3^{5} \cdot 3^{1+2}, 3 \times 3^{1+6} \cdot 3^{2}\right\} \subseteq \mathcal{R}_{0}\left(M_{5}, 2\right),
$$

and hence $N_{M_{5}}(R) \leqslant N_{M_{5}}\left(3^{6}\right)$ for all $R \in \mathcal{R}_{0}(L, 3)$. Let $R \in \mathcal{R}_{0}(L, 3) \backslash\left\{3^{6}\right\}$ and $\sigma(R): 1<3<Q=3^{6}<R$, so that $\sigma(R)^{\prime}: 1<3<R$. A similar proof to that of Case (1) shows that we may suppose that

$$
C \notin \bigcup_{R \in \mathcal{R}_{0}(L, 3) \backslash\left\{3^{6}\right\}}\left(\mathcal{R}^{-}\left(\sigma(R), 3^{6}\right) \cup \mathcal{R}^{0}\left(\sigma(R), 3^{6}\right)\right) .
$$

In particular, if $P_{1}=3$, then $P_{2} \notin_{G} \mathcal{R}_{0}(L, 3) \backslash\left\{3^{6}\right\}$; moreover, if $P_{2}=3^{6}$, then $C=C(17)$.

Let $H=N_{M_{5}}\left(3^{2}\right)=S_{3} \times\left(S_{3} \times U_{4}(3): 2\right) .2$. We may take

$$
\mathcal{R}_{0}(H, 3)=\left\{3^{2}, 3^{6}, 3^{2} \times 3^{1+4}, 3 \times 3^{2} \cdot 3^{2} \cdot 3^{3}\right\} ;
$$

moreover, $C_{M_{5}}\left(3^{6}\right)=3^{6}, C_{M_{5}}\left(3^{2} \times 3^{1+4}\right)=C_{M_{5}}\left(3 \times 3^{2} \cdot 3^{2} \cdot 3^{3}\right)=3^{3}$, and

$$
N_{H}(R)= \begin{cases}S_{3} \times S_{3} \times 3^{4} \cdot 2 S_{6} & \text { if } R=3^{6}, \\ S_{3} \times S_{3} \times 3^{1+4} \cdot 2_{-}^{1+4} \cdot S_{3} & \text { if } R=3^{2} \times 3^{1+4}, \\ S_{3} \times 3^{2} \cdot 3^{2} \cdot 3^{3} \cdot 2^{3} \cdot 2^{2} & \text { if } R=3 \times 3^{2} \cdot 3^{2} \cdot 3^{3} .\end{cases}
$$

Let $C^{\prime}: 1<3<3^{2}<3 \times 3^{2} \cdot 3^{2} \cdot 3^{3}$ and $g\left(C^{\prime}\right): 1<3<3^{2}<3^{2} \times 3^{1+4}<3 \times 3^{2} \cdot 3^{2} \cdot 3^{3}$. Then $N\left(C^{\prime}\right)=N\left(g\left(C^{\prime}\right)\right)=N_{H}\left(3 \times 3^{2} \cdot 3^{2} \cdot 3^{3}\right)$ and (6.2) holds. It follows that if $P_{1}=3$ and $P_{2}=3^{2}$, then we may suppose that $C \in_{G}\{C(13), C(14), C(15), C(16)\}$. 
Table 6: Some radical 3-chains of $\mathbb{B}$.

\begin{tabular}{|c|c|c|}
\hline$C$ & & $N(C)$ \\
\hline$C(1)$ & 1 & $\mathbb{B}$ \\
\hline$C(2)$ & $1<3_{+}^{1+8}$ & $3_{+}^{1+8}: 2_{-}^{1+6} \cdot U_{4}(2) .2$ \\
\hline$C(3)$ & $1<3^{2} \cdot 3^{3} \cdot 3^{6}<3_{+}^{1+8} \cdot 3^{3}$ & $3_{+}^{1+8} \cdot 3^{3} \cdot 2^{4} \cdot S_{3}$ \\
\hline$C(4)$ & $1<3^{2} \cdot 3^{3} \cdot 3^{6}$ & $3^{2} \cdot 3^{3} \cdot 3^{6} \cdot\left(S_{4} \times 2 S_{4}\right)$ \\
\hline$C(5)$ & $1<3^{3} \cdot 3^{6}<3^{3} \cdot 3^{6} \cdot 3^{2}$ & $3^{3} \cdot 3^{6} \cdot 3^{2}\left(2 S_{4} \times D_{8}\right)$ \\
\hline$C(6)$ & $1<3^{3} \cdot 3^{6}$ & $3^{3} \cdot 3^{6} \cdot\left(L_{3}(3) \times D_{8}\right)$ \\
\hline$C(7)$ & $1<3^{3} \cdot 3^{6}<3_{+}^{1+8} \cdot 3^{2}$ & $3_{+}^{1+8} \cdot 3^{2}\left(2 S_{4} \times D_{8}\right)$ \\
\hline$C(8)$ & $1<3^{3} \cdot 3^{6}<3_{+}^{1+8} \cdot 3^{2}<3_{+}^{1+8} \cdot 3^{2} \cdot 3$ & $3_{+}^{1+8} \cdot 3^{2} \cdot 3\left(2^{2} \times D_{8}\right)$ \\
\hline$C(9)$ & $1<3^{3} \cdot 3 \cdot 3^{3} \cdot 3^{3}<3^{2} \cdot 3^{3} \cdot 3^{6} \cdot 3$ & $3^{2} \cdot 3^{3} \cdot 3^{6} \cdot 3\left(2 \times 2 S_{4}\right)$ \\
\hline$C(10)$ & $1<3^{3} \cdot 3 \cdot 3^{3} \cdot 3^{3}$ & $3^{3} \cdot 3 \cdot 3^{3} \cdot 3^{3}\left(L_{3}(3) \times 2\right)$ \\
\hline$C(11)$ & $1<3^{3} \cdot 3 \cdot 3^{3} \cdot 3^{3}<3_{+}^{1+8} \cdot 3_{+}^{1+2}$ & $3_{+}^{1+8} \cdot 3_{+}^{1+2}\left(2 \times 2 S_{4}\right)$ \\
\hline$C(12)$ & $1<3^{3} \cdot 3 \cdot 3^{3} \cdot 3^{3}<3^{2} \cdot 3^{3} \cdot 3^{6} \cdot 3<S$ & $S .2^{3}$ \\
\hline$C(13)$ & $1<3<3^{2}$ & $S_{3} \times\left(S_{3} \times U_{4}(3): 2\right) .2$ \\
\hline$C(14)$ & $1<3<3^{2}<3^{2} \times 3_{+}^{1+4}$ & $S_{3} \times S_{3} \times 3_{+}^{1+4} \cdot 2_{-}^{1+4} \cdot S_{3}$ \\
\hline$C(15)$ & $1<3<3^{2}<3^{6}<3 \times 3^{2} \cdot 3^{2} \cdot 3^{3}$ & $S_{3} \times 3^{2} \cdot 3^{2} \cdot 3^{3} \cdot 2^{3} \cdot 2^{2}$ \\
\hline$C(16)$ & $1<3<3^{2}<3^{6}$ & $S_{3} \times S_{3} \times 3^{4}: 2 S_{6}$ \\
\hline$C(17)$ & $1<3<3^{6}$ & $S_{3} \times 3^{5}:\left(2 \times U_{4}(2): 2\right)$ \\
\hline$C(18)$ & $1<3$ & $S_{3} \times \mathrm{Fi}_{22}: 2$ \\
\hline$C(19)$ & $1<3<3 \times 3^{3+3}$ & $S_{3} \times 3^{3+3}: L_{3}(3)$ \\
\hline$C(20)$ & $1<3<3 \times 3^{3+3}<3 \times 3^{5}: 3_{+}^{1+2}$ & $S_{3} \times 3^{5}: 3_{+}^{1+2} \cdot 2 S_{4}$ \\
\hline$C(21)$ & $1<3<3 \times 3_{+}^{1+6}$ & $S_{3} \times 3_{+}^{1+6} \cdot 2^{3+4}: 3^{2} \cdot 2^{2}$ \\
\hline$C(22)$ & $1<3<3 \times 3_{+}^{1+6}<3 \times 3_{+}^{1+6} .3$ & $S_{3} \times 3_{+}^{1+6} \cdot 3: 2 S_{4}$ \\
\hline$C(23)$ & $1<3<3 \times 3_{+}^{1+6}<3 \times 3_{+}^{1+6} .3<3 \times 3_{+}^{1+6} .3^{2}$ & $S_{3} \times 3_{+}^{1+6} \cdot 3^{2} \cdot 2^{2}$ \\
\hline$C(24)$ & $1<3<3 \times 3_{+}^{1+6}<3 \times 3^{5} .3^{3}$ & $S_{3} \times 3^{5} \cdot 3^{3} \cdot\left(2 \times S_{4}\right) \cdot 2$ \\
\hline$C(25)$ & $1<3^{2}<3^{6}$ & $\left(3^{2}: D_{8} \times 3^{4}: A_{6}: 2^{2}\right) .2$ \\
\hline$C(26)$ & $1<3^{2}<3^{6}<3^{3} \cdot 3^{2} \cdot 3^{3}$ & $3^{3} \cdot 3^{2} \cdot 3^{3} \cdot 2^{3} \cdot 2^{2} \cdot 2^{3}$ \\
\hline$C(27)$ & $1<3^{2}<3^{2} \times 3_{+}^{1+4}$ & $\left(3^{2}: D_{8} \times 3_{+}^{1+4} \cdot 2 S_{4}: 2^{2}\right) \cdot 2$ \\
\hline$C(28)$ & $1<3^{2}$ & $\left(3^{2}: D_{8} \times U_{4}(3): 2^{2}\right) .2$ \\
\hline$C(29)$ & $1<3^{6}<3^{3+6}$ & $3^{3+6} \cdot\left(2^{2} \times L_{3}(3)\right)$ \\
\hline$C(30)$ & $1<3^{6}<3^{3+6}<3^{2} \cdot 3^{3} \cdot 3^{6}$ & $3^{2} \cdot 3^{3} \cdot 3^{6} \cdot\left(2^{2} \times 2 S_{4}\right)$ \\
\hline$C(31)$ & $1<3^{6}<3^{3+6}<3^{2} \cdot 3^{3} \cdot 3^{6}<3^{3+6} \cdot 3_{+}^{1+2}$ & $3^{3+6} \cdot 3_{+}^{1+2} \cdot 2^{4}$ \\
\hline$C(32)$ & $1<3^{6}<3^{3+6}<3^{6} \cdot 3^{2+3}$ & $3^{6} \cdot 3^{2+3} \cdot\left(2^{2} \times 2 S_{4}\right)$ \\
\hline$C(33)$ & $1<3^{6}<3_{+}^{1+8} .3$ & $3_{+}^{1+8} \cdot 3 \cdot 2^{2} \cdot 2^{4} \cdot 3^{2} \cdot D_{8}$ \\
\hline$C(34)$ & $1<3^{6}$ & $3^{6}:\left(2 \times L_{4}(3): 2\right): 2$ \\
\hline$C(35)$ & $1<3^{6}<3^{2} \cdot 3^{3} \cdot 3^{6}$ & $3^{2} \cdot 3^{3} \cdot 3^{6} \cdot\left(2 \times 2 S_{4}: 2\right): 2$ \\
\hline$C(36)$ & $1<3^{6}<3^{2} \cdot 3^{3} \cdot 3^{6}<3^{3+6} \cdot 3_{+}^{1+2}$ & $3^{3+6} \cdot 3_{+}^{1+2} \cdot 2^{3} \cdot 2^{2}$ \\
\hline
\end{tabular}


Let $J=N_{M_{5}}\left(3 \times 3^{3+3}\right)=S_{3} \times 3^{3+3}: L_{3}(3)$. We may take

$$
\mathcal{R}_{0}(J, 3)=\left\{3 \times 3^{3+3}, 3 \times 3^{1+6} .3,3 \times 3^{5}: 3^{1+2}, 3 \times 3^{1+6} .3^{2}\right\} \subseteq \mathcal{R}_{0}\left(M_{5}, 3\right) ;
$$

moreover, $N_{J}\left(3 \times 3^{1+6} .3\right)=N_{M_{5}}\left(3 \times 3^{1+6} .3\right)$, and

$$
N_{J}(R)= \begin{cases}S_{3} \times 3^{5}: 3^{1+2} \cdot 2 S_{4} & \text { if } R=3 \times 3^{5}: 3^{1+2}, \\ S_{3} \times 3^{1+6} \cdot 3^{2} \cdot 2^{2} & \text { if } R=3 \times 3^{1+6} \cdot 3^{2}\end{cases}
$$

Let $\sigma: 1<3<Q=3 \times 3^{3+3}<3 \times 3^{1+6} .3$, so that $\sigma^{\prime}: 1<3<3 \times 3^{1+6}$.3. A similar proof to that of Case (1) shows that we may suppose that

$$
C \notin\left(\mathcal{R}^{-}\left(\sigma, 3 \times 3^{3+3}\right) \cup \mathcal{R}^{0}\left(\sigma, 3 \times 3^{3+3}\right)\right) .
$$

Let $C^{\prime}: 1<3<3 \times 3^{3+3}<3 \times 3^{1+6} .3^{2}$ and $g\left(C^{\prime}\right): 1<3<3 \times 3^{3+3}<3 \times 3^{5}: 3^{1+2}<$ $3 \times 3^{1+6} \cdot 3^{2}$. Then $N\left(C^{\prime}\right)=N\left(g\left(C^{\prime}\right)\right)=N_{J}\left(3 \times 3^{1+6} \cdot 3^{2}\right)$ and (6.2) holds. It follows that if $P_{1}=3$ and $P_{2}=3 \times 3^{3+3}$, then we may suppose that $C \in_{G}\{C(19), C(20)\}$.

Let $K=N_{M_{5}}\left(3 \times 3^{1+6}\right)=S_{3} \times 3^{1+6} \cdot 2^{3+4}: 3^{2} \cdot 2^{2}$. We may take

$$
\mathcal{R}_{0}(K, 3)=\left\{3 \times 3^{1+6}, 3 \times 3^{1+6} .3,3 \times 3^{5} .3^{3}, 3 \times 3^{1+6} .3^{2}\right\} \subseteq \mathcal{R}_{0}\left(M_{5}, 3\right) ;
$$

moreover, $N_{K}(R)=N_{M_{5}}(R)$ for each $R \in \mathcal{R}_{0}(K, 3)$.

Let $C^{\prime}: 1<3<3 \times 3^{1+6}<3 \times 3^{1+6} .3^{2}$ and $g\left(C^{\prime}\right): 1<3<3 \times 3^{1+6}<3 \times 3^{5} .3^{3}<$ $3 \times 3^{1+6} \cdot 3^{2}$. Then $N\left(C^{\prime}\right)=N\left(g\left(C^{\prime}\right)\right)=N_{K}\left(3 \times 3^{1+6} \cdot 3^{2}\right)$ and (6.2) holds. If $P_{1}=3$ and $P_{2}=3 \times 3^{1+6}$, then we may suppose that $C \in_{G}\{C(21), C(22), C(23), C(24)\}$.

It follows that if $P_{1}=3$, then $C={ }_{G} C(i)$ for $13 \leqslant i \leqslant 24$

Case (2.6) Let $\sigma: 1<3^{2}<Q=3^{2} \times 3^{1+4}<3^{3} \cdot 3^{2} \cdot 3^{3}$, so that $\sigma^{\prime}: 1<3^{2}<3^{3} \cdot 3^{2} \cdot 3^{3}$, where $3^{3} \cdot 3^{2} \cdot 3^{3}, 3^{2} \times 3^{1+4} \in \mathcal{R}_{0}\left(M_{6}, 3\right)$. A similar proof to that of Case (1) shows that we may suppose that

$$
C \notin\left(\mathcal{R}^{-}\left(\sigma, 3^{2} \times 3^{1+4}\right) \cup \mathcal{R}^{0}\left(\sigma, 3^{2} \times 3^{1+4}\right)\right) .
$$

In particular, if $P_{1}=3^{2}$, then $P_{2} \neq_{G} 3^{3} \cdot 3^{2} \cdot 3^{3}$; if, moreover, $P_{2}=3^{2} \times 3^{1+4}$, then $P_{3} \neq \neq_{G} 3^{3} .3^{2} .3^{3}$. Thus we may suppose that $C \in_{G}\{C(25), C(26), C(27), C(28)\}$.

Case (2.7) Let $T=N_{M_{7}}\left(3^{1+8} \cdot 3\right)=N\left(3^{1+8} \cdot 3\right)=3^{1+8} \cdot 3 \cdot 2^{2} \cdot 2^{4} \cdot 3^{2} \cdot D_{8}$. We may take

$$
\mathcal{R}_{0}(T, 3)=\left\{3^{1+8} .3,3^{6} \cdot 3^{2+3}, 3^{3+6} \cdot 3^{1+2}\right\} \subseteq \mathcal{R}_{0}\left(M_{7}, 3\right) ;
$$

moreover, $N_{T}(R)=N_{M_{7}}(R)$ for each $R \in \mathcal{R}_{0}(T, 3)$.

Let $R \in \mathcal{R}_{0}(T, 3) \backslash\left\{3^{1+8} .3\right\}, \sigma(R): 1<3^{6}<Q=3^{1+8} .3<R$, so that $\sigma(R)^{\prime}: 1<$ $3^{6}<R$. A similar proof to that of Case (1) shows that we may suppose that

$$
C \notin \bigcup_{R \in \mathcal{R}_{0}(T, 3) \backslash\left\{3^{1+8} .3\right\}}\left(\mathcal{R}^{-}\left(\sigma(R), 3^{1+8} .3\right) \cup \mathcal{R}^{0}\left(\sigma, 3^{1+8} .3\right)\right) .
$$

In particular, if $P_{1}=3^{6}$, then $P_{2} \notin \mathcal{R}_{0}(T, 3) \backslash\left\{3^{1+8} .3\right\}$; if, moreover, $P_{2}=3^{1+8} .3$, then $P_{3} \notin \mathcal{R}_{0}(T, 3)$.

Let $V=N_{M_{7}}\left(3^{3+6}\right)=3^{3+6} \cdot\left(2^{2} \times L_{3}(3)\right)$. We may take

$$
\mathcal{R}_{0}(V, 3)=\left\{3^{3+6}, 3^{2} \cdot 3^{3} \cdot 3^{6}, 3^{6} \cdot 3^{2+3}, 3^{3+6} \cdot 3^{1+2}\right\} \subseteq \mathcal{R}_{0}\left(M_{7}, 3\right) ;
$$

moreover,

$$
N_{V}(R)= \begin{cases}3^{2} \cdot 3^{3} \cdot 3^{6} \cdot\left(2^{2} \times 2 S_{4}\right) & \text { if } R=3^{2} \cdot 3^{3} \cdot 3^{6} \\ 3^{6} \cdot 3^{2+3} \cdot\left(2^{2} \times 2 S_{4}\right) & \text { if } R=3^{6} \cdot 3^{2+3} \\ 3^{3+6} \cdot 3^{1+2} \cdot 2^{4} & \text { if } R=3^{3+6} \cdot 3^{1+2}\end{cases}
$$


Let $C^{\prime}: 1<3^{6}<3^{3+6}<3^{6} .3^{2+3}<3^{3+6} .3^{1+2}$ and $g\left(C^{\prime}\right): 1<3^{6}<3^{3+6}<3^{3+6} \cdot 3^{1+2}$. Then $N\left(C^{\prime}\right)=N\left(g\left(C^{\prime}\right)\right)=N_{V}\left(3^{3+6} .3^{1+2}\right)$ and (6.2) holds. If $P_{1}=3^{6}$ and $P_{2}=3^{3+6}$, then we may suppose that $C \in_{G}\{C(29), C(30), C(31), C(32)\}$.

Since Sylow 3 -subgroups of $N_{M_{7}}\left(3^{2} \cdot 3^{3} \cdot 3^{6}\right)=3^{2} \cdot 3^{3} \cdot 3^{6} \cdot\left(2 \times 2 S_{4}: 2\right): 2$ are the only radical subgroups of $N_{M_{7}}\left(3^{2} \cdot 3^{3} \cdot 3^{6}\right)$ other than $3^{2} \cdot 3^{3} \cdot 3^{6}$, it follows that if $P_{1}=3^{6}$, then $C={ }_{G} C(i)$ for some $29 \leqslant i \leqslant 36$.

REMARK. Let $\hat{G}=2 \cdot \mathbb{B}$ and let $\xi$ be the faithful character of $\operatorname{Irr}(Z(\hat{G}))$ and $\hat{B} \in \operatorname{Blk}(\hat{G})$ covering the block of $B(\xi)$. If $D(\hat{B}) \neq 1$ and $p=3,5$, then

$$
\sum_{C \in \mathcal{R}(G) / G}(-1)^{|C|} \mathrm{k}\left(N_{\hat{G}}(C), \hat{B}, d, \xi,[r]\right)=\sum_{C \in \mathcal{R}^{0}(G) / G}(-1)^{|C|} \mathrm{k}\left(N_{\hat{G}}(C), \hat{B}, d, \xi,[r]\right) .
$$

The proof of the remark is the same as that of Lemma 6.1, since $N\left(C^{\prime}\right)=N\left(g\left(C^{\prime}\right)\right)$ implies that $N_{\hat{G}}\left(C^{\prime}\right)=N_{\hat{G}}\left(g\left(C^{\prime}\right)\right)$.

\section{The proof of Uno's projective conjecture}

Lemma 7.1. Let $G$ be a finite group, and let $B \in \operatorname{Blk}(G)$ with $\operatorname{plr}(B)=2$ and abelian defect group $D=D(B)$. Let $O_{p}(G) \neq R<D$ be radical, and let $b \in B l k\left(N_{G}(R)\right)$ with $b^{G}=B$. Then

$$
\mathrm{k}\left(N_{G}(R) \cap N_{G}(D), b, d, \rho,[r]\right)=\mathrm{k}\left(N_{G}(R), b, d, \rho,[r]\right) .
$$

Proof. Since $R$ is radical and $D$ is abelian, $D$ is a defect group of $b, \operatorname{plr}(b) \neq 0$ and $\operatorname{plr}(b)=1$. By [3], we see that [3, Conjecture 1.3] holds for $b$; that is,

$$
\begin{aligned}
\mathrm{k}\left(N_{G}(R), b, d, \rho,[r]\right)-\mathrm{k}\left(N_{G}(R) \cap N_{G}(D), b, d, \rho,[r]\right) \\
\quad=w\left(N_{G}(R), b, d, \rho,[r], R\right)-w\left(N_{G}(R) \cap N_{G}(D), b, d, \rho,[r], R\right),
\end{aligned}
$$

where $w(H, Q)$ is the number of irreducible characters of $H$ afforded by a $Q$-projective $\mathcal{O H}$-module. By [13], if

$$
w\left(N_{G}(R), b, d, \rho,[r], R\right) \neq 0 \quad \text { or } \quad w\left(N_{G}(R) \cap N_{G}(D), b, d, \rho,[r], R\right) \neq 0,
$$

then $C_{D}(R) \leqslant R$, which is impossible. Thus $w\left(N_{G}(R) \cap N_{G}(D), b, d, \rho,[r], R\right)=$ $w\left(N_{G}(R), b, d, \rho,[r], R\right)=0$ and (7.1) holds.

Suppose that $\hat{G}=2 \cdot \mathbb{B}$, and that $B \in \operatorname{Blk}(\hat{G})$ with $D(B) \cong p^{2}$, so that $\operatorname{plr}(B)=2$. Thus Uno's projective conjecture for $B$ is equivalent to the equation

$$
\mathrm{k}(2 . \mathbb{B}, B, d, \rho,[r])=\mathrm{k}\left(N_{2 \cdot \mathbb{B}}(D(B)), B, d, \rho,[r]\right) .
$$

Note that if $\rho$ is the trivial character, then $B$ is a block of $\mathbb{B}$.

The tables listing the degrees of the irreducible characters referred to in the proof of Theorems 7.2 and 7.3 are available in Appendix A.

THEOREM 7.2. Let $B$ be a p-block of the Baby Monster $G=\mathbb{B}$ with a positive defect. If $p$ is odd, then B satisfies Uno's ordinary conjecture.

Proof. We may suppose that $D(B)$ is non-cyclic; by Lemma 5.2, $B \in\left\{B_{0}, B_{1}, B_{2}\right\}$ when $p=7,5$ and $B \in\left\{B_{0}, B_{1}, B_{2}, B_{3}\right\}$ when $p=3$. 
Case (1) If $p=7$, then $D(B) \cong 7^{2}, N_{\mathbb{B}}(D(B)) \cong\left(2^{2} \times 7^{2}:\left(3 \times 2 A_{4}\right)\right): 2$ and

$$
\mathrm{k}(\mathbb{B}, B, d,[r])=\mathrm{k}(N(D(B)), B, d,[r])= \begin{cases}9 & \text { if } d=2 \text { and } r=1, \\ 9 & \text { if } d=2 \text { and } r=2, \\ 9 & \text { if } d=2 \text { and } r=3, \\ 0 & \text { otherwise. }\end{cases}
$$

Thus (7.2) holds.

Case (2) Suppose that $p=5$ and $B=B_{1}$ or $B_{2}$. Then $D(B) \cong 5^{2}, N(D(B))=$ $N(C(10)) \cong 5^{2}: 4 S_{4} \times S_{5}$ and Theorem 7.2 follows because

$$
\mathrm{k}(\mathbb{B}, B, d,[r])=\mathrm{k}(N(C(10)), B, d,[r])= \begin{cases}10 & \text { if } d=2 \text { and } r=1, \\ 10 & \text { if } d=2 \text { and } r=2, \\ 0 & \text { otherwise. }\end{cases}
$$

Suppose that $B=B_{0}$. Since $N_{\mathbb{B}}(C(3)) \cong 5: 4 \times 5: 4 \times S_{5}$, its principal block $b_{0}=$ $B_{0}(N(C(3)))$ has $p$-local rank one and $N(C(4))=N\left(D\left(b_{0}\right)\right)$. Thus (7.1) holds for $b=b_{0}$ (with $\rho=1)$. Similarly, (7.1) holds for $b_{0}=B_{0}(N(C(10)))$ with $N(C(9))=N\left(D\left(b_{0}\right)\right)$, since $N(C(10)) \cong 5^{2}: 4 S_{4} \times S_{5}$.

We set $\mathrm{k}(i, d, r)=\mathrm{k}(N(C(i)), B, d,[r])$ for integers $i, d$ and $r$. The values $\mathrm{k}(i, d, r)$ are given in Table 7.

It follows that

$$
\sum_{i=1}^{10}(-1)^{|C(i)|} \mathrm{k}\left(N(C(i)), B_{0}, d,[r]\right)=0
$$

Case (3) Suppose that $p=3$, so that Uno's projective conjecture is equivalent to Dade's projective conjecture.

If $B=B_{1}, B_{2}$ or $B_{3}$, then $N(D(B))=N(C(28)) \cong\left(3^{2}: D_{8} \times U_{4}(3): 2^{2}\right) .2$ and Theorem 7.2 follows because

$$
\mathrm{k}(\mathbb{B}, B, d)=\mathrm{k}(N(C(28)), B, d)= \begin{cases}9 & \text { if } d=2 \\ 0 & \text { otherwise }\end{cases}
$$

Table 7: Values of $\mathrm{k}(i, d, r)$ when $p=5$ and $B=B_{0}$.

\begin{tabular}{l|rrrrrrrc}
\hline Defect $d$ & 6 & 5 & 5 & 4 & 4 & 3 & 3 & otherwise \\
Value $r$ & 1 & 1 & 2 & 1 & 2 & 1 & 2 & otherwise \\
\hline $\mathrm{k}(1, d, r)$ & 25 & 22 & 22 & 1 & 0 & 6 & 3 & 0 \\
$\mathrm{k}(2, d, r)=\mathrm{k}(5, d, r)$ & 0 & 0 & 0 & 50 & 50 & 5 & 20 & 0 \\
$\mathrm{k}(6, d, r)$ & 25 & 8 & 4 & 1 & 0 & 1 & 0 & 0 \\
$\mathrm{k}(7, d, r)$ & 25 & 8 & 4 & 4 & 4 & 1 & 0 & 0 \\
$\mathrm{k}(8, d, r)$ & 25 & 22 & 22 & 4 & 4 & 6 & 3 & 0 \\
\hline
\end{tabular}


Suppose that $B=B_{0}$; suppose also that $C \in \mathcal{R}^{0}$ with $\mathrm{d}(N(C))=8$, so that $C={ }_{G} C(i)$ for $13 \leqslant i \leqslant 16$ or $25 \leqslant i \leqslant 28$. Set $\mathrm{k}(i, d)=\mathrm{k}(N(C(i)), B, d)$. The values $\mathrm{k}(i, d)$ are given in Table 8.

It follows that

$$
\sum_{\mathrm{d}(N(C))=8}(-1)^{|C|} \mathrm{k}\left(N(C), B_{0}, d\right)=0 .
$$

Suppose that $C \in \mathcal{R}^{0}$ with $\mathrm{d}(N(C))=10$, so that $C={ }_{G} C(i)$ for $17 \leqslant i \leqslant 24$. The values $\mathrm{k}(i, d)$ are given in Table 9 .

It follows that

$$
\sum_{\mathrm{d}(N(C))=10}(-1)^{|C|} \mathrm{k}\left(N(C), B_{0}, d\right)=0 .
$$

Table 8: Values of $\mathrm{k}(i, d)$ when $p=3$ and $\mathrm{d}(N(C(i)))=8$.

\begin{tabular}{l|rrrrc}
\hline Defect $d$ & 8 & 7 & 6 & 5 & otherwise \\
\hline $\mathrm{k}(13, d)$ & 243 & 108 & 108 & 36 & 0 \\
$\mathrm{k}(14, d)$ & 243 & 108 & 135 & 36 & 0 \\
$\mathrm{k}(15, d)$ & 243 & 108 & 135 & 0 & 0 \\
$\mathrm{k}(16, d)$ & 243 & 108 & 108 & 0 & 0 \\
$\mathrm{k}(25, d)$ & 162 & 54 & 63 & 0 & 0 \\
$\mathrm{k}(26, d)$ & 162 & 54 & 81 & 0 & 0 \\
$\mathrm{k}(27, d)$ & 162 & 81 & 81 & 27 & 0 \\
$\mathrm{k}(28, d)$ & 162 & 81 & 63 & 27 & 0 \\
\hline
\end{tabular}

Table 9: Values of $\mathrm{k}(i, d)$ when $p=3$ and $\mathrm{d}(N(C(i)))=10$.

\begin{tabular}{l|rrrrrrc}
\hline Defect $d$ & 10 & 9 & 8 & 7 & 6 & 5 & otherwise \\
\hline $\mathrm{k}(17, d)$ & 81 & 117 & 114 & 9 & 36 & 0 & 0 \\
$\mathrm{k}(18, d)$ & 81 & 117 & 39 & 9 & 36 & 12 & 0 \\
$\mathrm{k}(19, d)$ & 54 & 36 & 39 & 9 & 0 & 0 & 0 \\
$\mathrm{k}(20, d)$ & 54 & 36 & 138 & 9 & 0 & 0 & 0 \\
$\mathrm{k}(21, d)$ & 81 & 144 & 39 & 54 & 45 & 12 & 0 \\
$\mathrm{k}(22, d)$ & 54 & 63 & 39 & 54 & 9 & 0 & 0 \\
$\mathrm{k}(23, d)$ & 54 & 63 & 138 & 54 & 0 & 0 & 0 \\
$\mathrm{k}(24, d)$ & 81 & 144 & 114 & 54 & 36 & 0 & 0 \\
\hline
\end{tabular}


Suppose that $C \in \mathcal{R}^{0}$ with $\mathrm{d}(N(C))=12$, so that $C={ }_{G} C(i)$ for $5 \leqslant i \leqslant 8$ or $29 \leqslant i \leqslant 36$. The values $\mathrm{k}(i, d)$ are given in Table 10 .

It follows that

$$
\sum_{\mathrm{d}(N(C))=12}(-1)^{|C|} \mathrm{k}\left(N(C), B_{0}, d\right)= \begin{cases}9 & \text { if } d=7 \\ 0 & \text { otherwise. }\end{cases}
$$

Suppose that $C \in \mathcal{R}^{0}$ with $\mathrm{d}(N(C))=13$, so $C={ }_{G} C(i)$ for $1 \leqslant i \leqslant 4$ or $9 \leqslant i \leqslant 12$. The values $\mathrm{k}(i, d)$ are given in Table 11 .

Table 10: Values of $\mathrm{k}(i, d)$ when $p=3$ and $\mathrm{d}(N(C(i)))=12$.

\begin{tabular}{l|rrrrrrrc}
\hline Defect $d$ & 12 & 11 & 10 & 9 & 8 & 7 & 6 & otherwise \\
\hline $\mathrm{k}(5, d)$ & 81 & 27 & 72 & 81 & 2 & 0 & 0 & 0 \\
$\mathrm{k}(6, d)$ & 81 & 27 & 36 & 24 & 2 & 0 & 0 & 0 \\
$\mathrm{k}(7, d)$ & 81 & 72 & 36 & 24 & 36 & 9 & 0 & 0 \\
$\mathrm{k}(8, d)$ & 81 & 72 & 72 & 81 & 36 & 0 & 0 & 0 \\
$\mathrm{k}(29, d)$ & 81 & 27 & 36 & 18 & 1 & 0 & 0 & 0 \\
$\mathrm{k}(30, d)$ & 81 & 27 & 81 & 69 & 1 & 0 & 0 & 0 \\
$\mathrm{k}(31, d)$ & 81 & 87 & 81 & 69 & 30 & 0 & 0 & 0 \\
$\mathrm{k}(32, d)$ & 81 & 87 & 36 & 18 & 30 & 9 & 0 & 0 \\
$\mathrm{k}(33, d)$ & 81 & 72 & 45 & 31 & 36 & 9 & 9 & 0 \\
$\mathrm{k}(34, d)$ & 81 & 27 & 45 & 31 & 2 & 0 & 9 & 0 \\
$\mathrm{k}(35, d)$ & 81 & 27 & 72 & 81 & 2 & 0 & 0 & 0 \\
$\mathrm{k}(36, d)$ & 81 & 72 & 72 & 81 & 36 & 0 & 0 & 0 \\
\hline
\end{tabular}

Table 11: Values of $\mathrm{k}(i, d)$ when $p=3$ and $\mathrm{d}(N(C(i)))=13$.

\begin{tabular}{l|rrrrrrrrrc}
\hline Defect $d$ & 13 & 12 & 11 & 10 & 9 & 8 & 7 & 6 & 5 & otherwise \\
\hline $\mathrm{k}(1, d)$ & 27 & 39 & 13 & 30 & 16 & 0 & 1 & 9 & 7 & 0 \\
$\mathrm{k}(2, d)$ & 27 & 39 & 38 & 30 & 34 & 16 & 13 & 9 & 7 & 0 \\
$\mathrm{k}(3, d)$ & 27 & 48 & 38 & 90 & 51 & 16 & 0 & 0 & 0 & 0 \\
$\mathrm{k}(4, d)$ & 27 & 48 & 13 & 90 & 36 & 0 & 0 & 0 & 0 & 0 \\
$\mathrm{k}(9, d)$ & 27 & 33 & 8 & 90 & 60 & 2 & 0 & 0 & 0 & 0 \\
$\mathrm{k}(10, d)$ & 27 & 24 & 8 & 48 & 18 & 2 & 1 & 0 & 0 & 0 \\
$\mathrm{k}(11, d)$ & 27 & 24 & 41 & 48 & 36 & 26 & 4 & 0 & 0 & 0 \\
$\mathrm{k}(12, d)$ & 27 & 33 & 41 & 90 & 75 & 26 & 0 & 0 & 0 & 0 \\
\hline
\end{tabular}


It follows that

$$
\sum_{\mathrm{d}(N(C))=13}(-1)^{|C|} \mathrm{k}\left(N(C), B_{0}, d\right)= \begin{cases}-9 & \text { if } d=7 \\ 0 & \text { otherwise }\end{cases}
$$

Theorem 7.2 follows for $B_{0}$.

THEOREM 7.3. Let $B$ be a p-block of the covering group $\hat{G}=2 . \mathbb{B}$ of the Baby Monster $\mathbb{B}$ with a positive defect. If $p$ is odd, then B satisfies Uno's projective conjecture.

Proof. We may suppose that $D(B)$ is non-cyclic, and that $\operatorname{Irr}(B) \nsubseteq \operatorname{Irr}(\mathbb{B})$, so that $B=\hat{B}$.

Case (1) If $p=7$, then $N_{2 \cdot \mathbb{B}}(D(B)) \cong 2 .\left(2^{2} \times 7^{2}:\left(3 \times 2 A_{4}\right)\right): 2$. The values of $\mathrm{k}(2 . \mathbb{B}, B, d, \xi,[r])$ and $\mathrm{k}\left(N_{2 . \mathbb{B}}(D(B)), B, d, \xi,[r]\right)$ are the same, and are given by (7.3).

Case (2) Suppose that $p=5$, so that $N_{2 \cdot \mathbb{B}}(C(i))$ has a unique block $\hat{b}(i)$ such that $\hat{b}(i)^{N_{2 . \mathbb{B}}(C(i))}=\hat{B}$. By Lemma 7.1, equation (7.1) holds for $b=\hat{b}(10)$ and $\hat{b}(3)$ (with $\rho=\xi)$. Set $\mathrm{k}(i, d, r)=\mathrm{k}\left(N_{2 \cdot \mathbb{B}}(C(i)), \hat{B}, d, \xi,[r]\right)$ for integers $i, d$ and $r$. The values $\mathrm{k}(i, d, r)$ are given in Table 12 .

Table 12: Values of $\mathrm{k}(i, d, r)$ when $p=5$ and $B=\hat{B}$.

\begin{tabular}{l|rrrrrrrc}
\hline Defect $d$ & 6 & 5 & 5 & 4 & 4 & 3 & 3 & otherwise \\
Value $r$ & 2 & 1 & 2 & 1 & 2 & 1 & 2 & otherwise \\
\hline $\mathrm{k}(1, d, r)$ & 25 & 5 & 12 & 0 & 1 & 5 & 1 & 0 \\
$\mathrm{k}(2, d, r)=\mathrm{k}(5, d, r)$ & 0 & 0 & 0 & 11 & 26 & 4 & 9 & 0 \\
$\mathrm{k}(6, d, r)$ & 25 & 4 & 8 & 0 & 1 & 0 & 1 & 0 \\
$\mathrm{k}(7, d, r)$ & 25 & 4 & 8 & 4 & 4 & 0 & 1 & 0 \\
$\mathrm{k}(8, d, r)$ & 25 & 5 & 12 & 4 & 4 & 5 & 1 & 0 \\
\hline
\end{tabular}

Table 13: Values of $\mathrm{k}(i, d)$ when $p=3$ and $\mathrm{d}\left(N_{2 \cdot \mathbb{B}}(C(i))\right)=8$.

\begin{tabular}{c|ccccc}
\hline Defect $d$ & 8 & 7 & 6 & 5 & otherwise \\
\hline $\mathrm{k}(13, d)$ & 87 & 18 & 27 & 12 & 0 \\
$\mathrm{k}(14, d)$ & 87 & 18 & 63 & 12 & 0 \\
$\mathrm{k}(15, d)$ & 87 & 42 & 63 & 0 & 0 \\
$\mathrm{k}(16, d)$ & 87 & 42 & 27 & 0 & 0 \\
$\mathrm{k}(25, d)$ & 57 & 18 & 18 & 0 & 0 \\
$\mathrm{k}(26, d)$ & 57 & 18 & 30 & 0 & 0 \\
$\mathrm{k}(27, d)$ & 57 & 12 & 30 & 6 & 0 \\
$\mathrm{k}(28, d)$ & 57 & 12 & 18 & 6 & 0 \\
\hline
\end{tabular}


It follows that

$$
\sum_{i=1}^{10}(-1)^{|C(i)|} \mathrm{k}\left(N_{2 . \mathbb{B}}(C(i)), \hat{B}, d, \xi,[r]\right)=0 .
$$

Case (3) Suppose that $p=3$, so that Uno's projective conjecture is equivalent to Dade's projective conjecture. Set $\mathrm{k}(i, d)=\mathrm{k}\left(N_{2 \cdot \mathbb{B}}(C(i)), \hat{B}, d, \xi\right)$.

Suppose that $C=C(i)$ is a chain with $\mathrm{d}\left(N_{2 \cdot \mathbb{B}}(C)\right)=8$. Then $13 \leqslant i \leqslant 16$ or $25 \leqslant i \leqslant 28$, and the values $\mathrm{k}(i, d)$ are given in Table 13 .

It follows that

$$
\sum_{\mathrm{d}\left(N_{2 . \mathbb{B}}(C)\right)=8}(-1)^{|C|} \mathrm{k}\left(N_{2 . \mathbb{B}}(C), \hat{B}, d, \xi\right)=0 .
$$

Suppose that $C=C(i)$ is a chain with $\mathrm{d}\left(N_{2 . \mathbb{B}}(C)\right)=10$. Then $17 \leqslant i \leqslant 24$ and the values $\mathrm{k}(i, d)$ are given in Table 14 .

It follows that

$$
\sum_{\mathrm{d}\left(N_{2 . \mathbb{B}}(C)\right)=10}(-1)^{|C|} \mathrm{k}\left(N_{2 . \mathbb{B}}(C), \hat{B}, d, \xi\right)=0 .
$$

Suppose that $C=C(i)$ is a chain with $\mathrm{d}\left(N_{2 . \mathbb{B}}(C)\right)=12$. Then $5 \leqslant i \leqslant 8$ or $29 \leqslant i \leqslant$ 36 , and the values $\mathrm{k}(i, d)$ are given in Table 15 .

It follows that

$$
\sum_{\mathrm{d}\left(N_{2, \mathbb{B}}(C)\right)=12}(-1)^{|C|} \mathrm{k}\left(N_{2 . \mathbb{B}}(C), \hat{B}, d, \xi\right)= \begin{cases}6 & \text { if } d=7, \\ 0 & \text { otherwise. }\end{cases}
$$

Suppose that $C=C(i)$ is a chain with $\mathrm{d}\left(N_{2 . \mathbb{B}}(C)\right)=13$. Then $1 \leqslant i \leqslant 4$ or $9 \leqslant i \leqslant 12$, and the values $\mathrm{k}(i, d)$ are given in Table 16 .

It follows that

$$
\sum_{\mathrm{d}\left(N_{2 . \mathbb{B}}(C)\right)=13}(-1)^{|C|} \mathrm{k}\left(N_{2 . \mathbb{B}}(C), \hat{B}, d, \xi\right)= \begin{cases}-6 & \text { if } d=7, \\ 0 & \text { otherwise. }\end{cases}
$$

Theorem 7.3 follows for $\hat{B}$.

Table 14: Values of $\mathrm{k}(i, d)$ when $p=3$ and $\mathrm{d}\left(N_{2 \cdot \mathbb{B}}(C(i))\right)=10$.

\begin{tabular}{l|rrrrrc}
\hline Defect $d$ & 10 & 9 & 8 & 7 & 6 & otherwise \\
\hline $\mathrm{k}(17, d, r)$ & 33 & 36 & 48 & 6 & 9 & 0 \\
$\mathrm{k}(18, d, r)$ & 33 & 36 & 3 & 6 & 9 & 0 \\
$\mathrm{k}(19, d, r)$ & 54 & 36 & 39 & 9 & 0 & 0 \\
$\mathrm{k}(20, d, r)$ & 54 & 36 & 138 & 9 & 0 & 0 \\
$\mathrm{k}(21, d, r)$ & 33 & 51 & 3 & 27 & 18 & 0 \\
$\mathrm{k}(22, d, r)$ & 54 & 63 & 39 & 54 & 9 & 0 \\
$\mathrm{k}(23, d, r)$ & 54 & 63 & 138 & 54 & 0 & 0 \\
$\mathrm{k}(24, d, r)$ & 33 & 51 & 48 & 27 & 9 & 0 \\
\hline
\end{tabular}


Table 15: Values of $\mathrm{k}(i, d)$ when $p=3$ and $\mathrm{d}\left(N_{2 \cdot \mathbb{B}}(C(i))\right)=12$.

\begin{tabular}{l|rrrrrrrc}
\hline Defect $d$ & 12 & 11 & 10 & 9 & 8 & 7 & 6 & otherwise \\
\hline $\mathrm{k}(5, d, r)$ & 36 & 12 & 36 & 54 & 2 & 0 & 0 & 0 \\
$\mathrm{k}(6, d, r)$ & 36 & 12 & 18 & 18 & 2 & 0 & 0 & 0 \\
$\mathrm{k}(7, d, r)$ & 36 & 36 & 18 & 18 & 24 & 6 & 0 & 0 \\
$\mathrm{k}(8, d, r)$ & 36 & 36 & 36 & 54 & 24 & 0 & 0 & 0 \\
$\mathrm{k}(29, d, r)$ & 36 & 12 & 27 & 12 & 1 & 0 & 0 & 0 \\
$\mathrm{k}(30, d, r)$ & 36 & 12 & 54 & 51 & 1 & 0 & 0 & 0 \\
$\mathrm{k}(31, d, r)$ & 36 & 51 & 54 & 51 & 24 & 0 & 0 & 0 \\
$\mathrm{k}(32, d, r)$ & 36 & 51 & 27 & 12 & 24 & 6 & 0 & 0 \\
$\mathrm{k}(33, d, r)$ & 36 & 36 & 18 & 10 & 24 & 6 & 3 & 0 \\
$\mathrm{k}(34, d, r)$ & 36 & 12 & 18 & 10 & 2 & 0 & 3 & 0 \\
$\mathrm{k}(35, d, r)$ & 36 & 12 & 36 & 54 & 2 & 0 & 0 & 0 \\
$\mathrm{k}(36, d, r)$ & 36 & 36 & 36 & 54 & 24 & 0 & 0 & 0 \\
\hline
\end{tabular}

Table 16: Values of $\mathrm{k}(i, d)$ when $p=3$ and $\mathrm{d}\left(N_{2 \cdot \mathbb{B}}(C(i))\right)=13$.

\begin{tabular}{l|rrrrrrrrrc}
\hline Defect $d$ & 13 & 12 & 11 & 10 & 9 & 8 & 7 & 6 & 5 & otherwise \\
\hline $\mathrm{k}(1, d)$ & 27 & 9 & 3 & 12 & 1 & 0 & 1 & 3 & 2 & 0 \\
$\mathrm{k}(2, d)$ & 27 & 9 & 14 & 12 & 19 & 6 & 10 & 3 & 2 & 0 \\
$\mathrm{k}(3, d)$ & 27 & 18 & 14 & 63 & 30 & 6 & 0 & 0 & 0 & 0 \\
$\mathrm{k}(4, d)$ & 27 & 18 & 3 & 63 & 15 & 0 & 0 & 0 & 0 & 0 \\
$\mathrm{k}(9, d)$ & 27 & 33 & 8 & 90 & 60 & 2 & 0 & 0 & 0 & 0 \\
$\mathrm{k}(10, d)$ & 27 & 24 & 8 & 48 & 18 & 2 & 1 & 0 & 0 & 0 \\
$\mathrm{k}(11, d)$ & 27 & 24 & 41 & 48 & 36 & 26 & 4 & 0 & 0 & 0 \\
$\mathrm{k}(12, d)$ & 27 & 33 & 41 & 90 & 75 & 26 & 0 & 0 & 0 & 0 \\
\hline
\end{tabular}

Acknowledgements. The authors would like to thank Thomas Breuer for constructing the character tables of a couple of the maximal subgroups of 2.BB. They also thank Eamonn O'Brien and the referee for valuable suggestions. 
Appendix A. Degrees of character tables for chain normalisers of $\mathbb{B}$ and $2 \mathbb{B}$

Let $\xi$ be the faithful linear character of $Z(2 . \mathbb{B})$, let $Z(2 . \mathbb{B}) \leqslant H \leqslant 2 . \mathbb{B}$, and let $\operatorname{Irr}(H \mid \xi)$ be the character of $\operatorname{Irr}(H)$ covering $\xi$.

Table A.1: The degrees of characters in $\operatorname{Irr}\left(3^{1+8} \cdot 2_{-}^{1+6} \cdot U_{4}(2) \cdot 2\right)$.

\begin{tabular}{l|rrrrrrrrr}
\hline Degree & 1 & 6 & 8 & 10 & 15 & 20 & 24 & 27 & 30 \\
Number & 2 & 2 & 2 & 1 & 4 & 3 & 2 & 2 & 2 \\
\hline Degree & 36 & 48 & 60 & 64 & 80 & 81 & 90 & 108 & 120 \\
Number & 4 & 2 & 3 & 2 & 2 & 2 & 1 & 2 & 4 \\
\hline Degree & 135 & 160 & 162 & 180 & 192 & 240 & 270 & 324 & 360 \\
Number & 4 & 3 & 2 & 8 & 2 & 2 & 5 & 4 & 4 \\
\hline Degree & 405 & 480 & 512 & 540 & 576 & 640 & 648 & 720 & 810 \\
Number & 2 & 3 & 2 & 2 & 2 & 1 & 2 & 1 & 2 \\
\hline Degree & 972 & 1296 & 1440 & 1620 & 2430 & 2880 & 3240 & 3888 & 4374 \\
Number & 1 & 1 & 4 & 2 & 2 & 4 & 1 & 1 & 1 \\
\hline Degree & 4860 & 5120 & 5760 & 5832 & 6480 & 7290 & 7776 & 8640 & 9720 \\
Number & 3 & 4 & 5 & 2 & 4 & 2 & 1 & 6 & 1 \\
\hline Degree & 10240 & 10368 & 11520 & 12960 & 13122 & 15360 & 17280 & 17496 & 19440 \\
Number & 2 & 1 & 4 & 6 & 3 & 4 & 7 & 1 & 2 \\
\hline Degree & 21870 & 23040 & 25920 & 29160 & 30720 & 31104 & 34560 & 38880 & 40960 \\
Number & 4 & 1 & 1 & 4 & 4 & 1 & 2 & 3 & 2 \\
\hline Degree & 43740 & 51840 & 52488 & 58320 & 61440 & 65610 & 77760 & 81920 & 82944 \\
Number & 2 & 3 & 2 & 4 & 5 & 1 & 1 & 1 & 1 \\
\hline Degree & 87480 & 93312 & 104976 & & & & & & \\
Number & 1 & 1 & 1 & & & & & & \\
\hline
\end{tabular}

Table A.2: The degrees of characters in $\operatorname{Irr}\left(2.3^{1+8} \cdot 2_{-}^{1+6} \cdot U_{4}(2) .2 \mid \xi\right)$.

\begin{tabular}{l|rrrrrrrrr}
\hline Degree & 8 & 20 & 40 & 60 & 64 & 72 & 80 & 108 & 120 \\
Number & 1 & 2 & 2 & 2 & 3 & 1 & 2 & 2 & 1 \\
\hline Degree & 160 & 288 & 320 & 432 & 480 & 512 & 540 & 576 & 640 \\
Number & 2 & 2 & 2 & 2 & 2 & 2 & 2 & 3 & 2 \\
\hline Degree & 648 & 720 & 960 & 3240 & 5184 & 5760 & 5832 & 9720 & 10240 \\
Number & 3 & 2 & 1 & 5 & 2 & 2 & 2 & 3 & 4 \\
\hline Degree & 10368 & 11520 & 12960 & 17280 & 17496 & 20480 & 23328 & 25920 & 34560 \\
Number & 1 & 4 & 1 & 2 & 1 & 2 & 4 & 5 & 3 \\
\hline Degree & 40960 & 46656 & 52488 & 61440 & 69120 & 69984 & 77760 & 81920 & 82944 \\
Number & 2 & 2 & 2 & 2 & 1 & 1 & 3 & 1 & 1 \\
\hline Degree & 87480 & 93312 & 103680 & 116640 & 122880 & & & & \\
Number & 1 & 1 & 1 & 1 & 1 & & & & \\
\hline
\end{tabular}


Table A.3: The degrees of characters in $\operatorname{Irr}\left(3^{1+8} \cdot 3^{3} \cdot 2^{4} \cdot S_{3}\right)$.

\begin{tabular}{l|rrrrrrrrrrrrrr}
\hline Degree & 1 & 2 & 3 & 4 & 6 & 8 & 12 & 16 & 18 & 24 & 32 & 36 & 48 & 54 \\
Number & 8 & 8 & 8 & 2 & 12 & 4 & 14 & 4 & 8 & 9 & 1 & 10 & 4 & 12 \\
\hline Degree & 72 & 96 & 108 & 144 & 162 & 216 & 324 & 432 & 486 & 648 & 864 & 972 & 1296 & 1944 \\
Number & 16 & 1 & 26 & 4 & 8 & 34 & 16 & 16 & 4 & 21 & 2 & 8 & 6 & 4 \\
\hline
\end{tabular}

Table A.4: The degrees of characters in $\operatorname{Irr}\left(2 \cdot 3^{1+8} \cdot 3^{3} \cdot 2^{4} \cdot S_{3} \mid \xi\right)$.

\begin{tabular}{l|rrrrrrrrrr}
\hline Degree & 2 & 4 & 8 & 12 & 16 & 24 & 32 & 36 & 48 & 72 \\
Number & 8 & 8 & 6 & 4 & 4 & 8 & 1 & 4 & 5 & 2 \\
\hline Degree & 96 & 108 & 144 & 216 & 324 & 432 & 648 & 864 & 1296 & 1944 \\
Number & 1 & 8 & 8 & 34 & 4 & 19 & 18 & 2 & 8 & 6 \\
\hline
\end{tabular}

Table A.5: The degrees of characters in $\operatorname{Irr}\left(3^{2} \cdot 3^{3} \cdot 3^{6}\left(S_{4} \times 2 S_{4}\right)\right)$.

\begin{tabular}{l|rrrrrrrrrrr}
\hline Degree & 1 & 2 & 3 & 4 & 6 & 8 & 9 & 12 & 24 & 32 & 48 \\
Number & 4 & 8 & 8 & 5 & 8 & 1 & 4 & 2 & 8 & 4 & 14 \\
\hline Degree & 54 & 64 & 96 & 108 & 128 & 144 & 162 & 192 & 216 & 288 & 324 \\
Number & 4 & 4 & 5 & 13 & 1 & 4 & 4 & 2 & 21 & 5 & 5 \\
\hline Degree & 384 & 432 & 648 & 864 & 1296 & 1728 & 2592 & 3456 & 5184 & & \\
Number & 1 & 19 & 6 & 21 & 11 & 10 & 8 & 2 & 2 & & \\
\hline
\end{tabular}

Table A.6: The degrees of characters in $\operatorname{Irr}\left(2 \cdot 3^{2} \cdot 3^{3} \cdot 3^{6}\left(S_{4} \times 2 S_{4}\right) \mid \xi\right)$.

\begin{tabular}{l|rrrrrrrrr}
\hline Degree & 2 & 4 & 6 & 8 & 12 & 16 & 32 & 48 & 64 \\
Number & 4 & 8 & 4 & 5 & 2 & 1 & 4 & 4 & 4 \\
\hline Degree & 96 & 108 & 128 & 192 & 216 & 288 & 324 & 384 & 432 \\
Number & 4 & 2 & 1 & 3 & 12 & 2 & 2 & 1 & 17 \\
\hline Degree & 576 & 648 & 864 & 1296 & 1728 & 2592 & 3456 & 5184 & \\
Number & 1 & 3 & 17 & 1 & 13 & 6 & 2 & 3 & \\
\hline
\end{tabular}


Table A.7: The degrees of characters in $\operatorname{Irr}\left(3^{3} \cdot 3^{6} \cdot 3^{2} \cdot\left(D_{8} \times 2 S_{4}\right)\right)$.

\begin{tabular}{l|rrrrrrrrr}
\hline Degree & 1 & 2 & 3 & 4 & 6 & 8 & 16 & 18 & 32 \\
Number & 8 & 14 & 8 & 7 & 2 & 9 & 22 & 4 & 17 \\
\hline Degree & 36 & 48 & 54 & 64 & 72 & 96 & 108 & 144 & 192 \\
Number & 17 & 4 & 4 & 4 & 19 & 9 & 9 & 11 & 4 \\
\hline Degree & 216 & 288 & 432 & 576 & 648 & 864 & 1152 & 1728 & 3456 \\
Number & 16 & 14 & 20 & 6 & 2 & 21 & 1 & 10 & 1 \\
\hline
\end{tabular}

Table A.8: The degrees of characters in $\operatorname{Irr}\left(2 \cdot 3^{3} \cdot 3^{6} \cdot 3^{2} \cdot\left(D_{8} \times 2 S_{4}\right) \mid \xi\right)$.

\begin{tabular}{l|rrrrrrrrrrr}
\hline Degree & 2 & 4 & 6 & 8 & 16 & 32 & 36 & 64 & 72 & 96 & 108 \\
Number & 4 & 6 & 4 & 2 & 4 & 14 & 2 & 6 & 11 & 2 & 2 \\
\hline Degree & 144 & 192 & 216 & 288 & 432 & 576 & 648 & 864 & 1152 & 1728 & 3456 \\
Number & 10 & 6 & 10 & 3 & 10 & 9 & 2 & 20 & 1 & 11 & 1 \\
\hline
\end{tabular}

Table A.9: The degrees of characters in $\operatorname{Irr}\left(3^{3} \cdot 3^{6} \cdot\left(L_{3}(3) \times D_{8}\right)\right)$.

\begin{tabular}{l|rrrrrrrrr}
\hline Degree & 1 & 2 & 12 & 13 & 16 & 24 & 26 & 27 & 32 \\
Number & 4 & 1 & 4 & 4 & 16 & 1 & 13 & 4 & 4 \\
\hline Degree & 39 & 52 & 54 & 78 & 104 & 156 & 208 & 234 & 416 \\
Number & 4 & 11 & 1 & 1 & 12 & 8 & 4 & 4 & 8 \\
\hline Degree & 468 & 624 & 702 & 832 & 936 & 1248 & 1404 & 1872 & 2496 \\
Number & 9 & 4 & 4 & 4 & 7 & 1 & 1 & 7 & 4 \\
\hline Degree & 2808 & 3744 & 5616 & 7488 & 8424 & 14976 & & & \\
Number & 8 & 6 & 6 & 2 & 2 & 1 & & & \\
\hline
\end{tabular}

Table A.10: The degrees of characters in $\operatorname{Irr}\left(2 \cdot 3^{3} \cdot 3^{6} \cdot\left(L_{3}(3) \times D_{8}\right) \mid \xi\right)$.

\begin{tabular}{l|rrrrrrrrrrrr}
\hline Degree & 2 & 24 & 26 & 32 & 52 & 54 & 78 & 104 & 208 & 312 & 468 & 832 \\
Number & 2 & 2 & 2 & 8 & 6 & 2 & 2 & 6 & 6 & 2 & 2 & 6 \\
\hline Degree & 936 & 1248 & 1404 & 1872 & 2496 & 2808 & 3744 & 5616 & 7488 & 8424 & 14976 & \\
Number & 5 & 2 & 2 & 4 & 4 & 8 & 3 & 6 & 3 & 2 & 1 & \\
\hline
\end{tabular}


Table A.11: The degrees of characters in $\operatorname{Irr}\left(3^{1+8} \cdot 3^{2} \cdot\left(2 S_{4} \times D_{8}\right)\right)$.

\begin{tabular}{l|rrrrrrrrrr}
\hline Degree & 1 & 2 & 3 & 4 & 6 & 8 & 12 & 16 & 32 & 48 \\
Number & 8 & 14 & 8 & 15 & 2 & 21 & 8 & 10 & 9 & 16 \\
\hline Degree & 64 & 72 & 96 & 144 & 162 & 192 & 288 & 324 & 384 & 432 \\
Number & 4 & 8 & 24 & 14 & 4 & 12 & 9 & 9 & 2 & 8 \\
\hline Degree & 486 & 576 & 648 & 864 & 972 & 1152 & 1296 & 1728 & 1944 & 2592 \\
Number & 4 & 4 & 13 & 14 & 1 & 1 & 9 & 2 & 4 & 1 \\
\hline
\end{tabular}

Table A.12: The degrees of characters in $\operatorname{Irr}\left(2 \cdot 3^{1+8} \cdot 3^{2} \cdot\left(2 S_{4} \times D_{8}\right) \mid \xi\right)$.

\begin{tabular}{l|rrrrrrrrrrrr}
\hline Degree & 2 & 4 & 6 & 8 & 16 & 24 & 32 & 64 & 96 & 144 & 192 & 288 \\
Number & 4 & 6 & 4 & 8 & 10 & 2 & 2 & 6 & 12 & 4 & 16 & 8 \\
\hline Degree & 324 & 384 & 576 & 648 & 864 & 972 & 1152 & 1296 & 1728 & 1944 & 2592 & \\
Number & 2 & 2 & 5 & 11 & 16 & 2 & 1 & 10 & 2 & 4 & 1 & \\
\hline
\end{tabular}

Table A.13: The degrees of characters in $\operatorname{Irr}\left(3^{1+8} \cdot 3^{2} \cdot 3 \cdot\left(2^{2} \times D_{8}\right)\right)$.

\begin{tabular}{l|rrrrrrrrrrrr}
\hline Degree & 1 & 2 & 4 & 6 & 8 & 12 & 16 & 18 & 24 & 36 & 48 & 54 \\
Number & 16 & 20 & 24 & 8 & 17 & 18 & 4 & 8 & 32 & 30 & 12 & 8 \\
\hline Degree & 72 & 96 & 108 & 144 & 162 & 216 & 288 & 324 & 432 & 648 & 864 & 1296 \\
Number & 25 & 2 & 18 & 8 & 8 & 42 & 1 & 10 & 12 & 16 & 1 & 2 \\
\hline
\end{tabular}

Table A.14: The degrees of characters in $\operatorname{Irr}\left(2 \cdot 3^{1+8} \cdot 3^{2} \cdot 3 \cdot\left(2^{2} \times D_{8}\right) \mid \xi\right)$.

\begin{tabular}{l|rrrrrrrrrr}
\hline Degree & 2 & 4 & 8 & 12 & 16 & 24 & 36 & 48 & 72 & 96 \\
Number & 8 & 8 & 14 & 4 & 6 & 12 & 4 & 18 & 20 & 2 \\
\hline Degree & 108 & 144 & 216 & 288 & 324 & 432 & 648 & 864 & 1296 & \\
Number & 4 & 11 & 34 & 1 & 4 & 15 & 18 & 1 & 2 & \\
\hline
\end{tabular}

Table A.15: The degrees of characters in $\operatorname{Irr}\left(3^{2} \cdot 3^{3} \cdot 3^{6} \cdot 3 \cdot\left(2 \times 2 S_{4}\right)\right)$.

\begin{tabular}{l|rrrrrrrrrrrr}
\hline Degree & 1 & 2 & 3 & 4 & 6 & 8 & 16 & 24 & 32 & 48 & 54 & 96 \\
Number & 4 & 8 & 4 & 5 & 2 & 5 & 4 & 16 & 1 & 10 & 20 & 1 \\
\hline Degree & 108 & 144 & 162 & 216 & 324 & 432 & 486 & 648 & 864 & 1296 & 2592 & \\
Number & 24 & 8 & 14 & 16 & 7 & 26 & 2 & 24 & 4 & 14 & 1 & \\
\hline
\end{tabular}


Table A.16: The degrees of characters in $\operatorname{Irr}\left(2 \times 3^{2} \cdot 3^{3} \cdot 3^{6} \cdot 3 \cdot\left(2 \times 2 S_{4}\right) \mid \xi\right)$.

\begin{tabular}{l|rrrrrrrrrrrr}
\hline Degree & 1 & 2 & 3 & 4 & 6 & 8 & 16 & 24 & 32 & 48 & 54 & 96 \\
Number & 4 & 8 & 4 & 5 & 2 & 5 & 4 & 16 & 1 & 10 & 20 & 1 \\
\hline Degree & 108 & 144 & 162 & 216 & 324 & 432 & 486 & 648 & 864 & 1296 & 2592 & \\
Number & 24 & 8 & 14 & 16 & 7 & 26 & 2 & 24 & 4 & 14 & 1 & \\
\hline
\end{tabular}

Table A.17: The degrees of characters in $\operatorname{Irr}\left(3^{3} \cdot 3 \cdot 3^{3} \cdot 3^{3} \cdot\left(L_{3}(3) \times 2\right)\right)$.

\begin{tabular}{l|rrrrrrrrr}
\hline Degree & 1 & 12 & 13 & 16 & 26 & 27 & 39 & 52 & 54 \\
Number & 2 & 2 & 2 & 8 & 8 & 2 & 2 & 3 & 1 \\
\hline Degree & 78 & 104 & 156 & 208 & 234 & 416 & 468 & 624 & 648 \\
Number & 8 & 1 & 6 & 2 & 2 & 1 & 3 & 6 & 1 \\
\hline Degree & 702 & 864 & 936 & 1404 & 1458 & 2106 & 4212 & 5616 & 6318 \\
Number & 13 & 4 & 3 & 16 & 1 & 11 & 6 & 12 & 2 \\
\hline
\end{tabular}

Table A.18: The degrees of characters in $\operatorname{Irr}\left(2 \times 3^{3} \cdot 3 \cdot 3^{3} \cdot 3^{3} \cdot\left(L_{3}(3) \times 2\right) \mid \xi\right)$.

\begin{tabular}{l|rrrrrrrrr}
\hline Degree & 1 & 12 & 13 & 16 & 26 & 27 & 39 & 52 & 54 \\
Number & 2 & 2 & 2 & 8 & 8 & 2 & 2 & 3 & 1 \\
\hline Degree & 78 & 104 & 156 & 208 & 234 & 416 & 468 & 624 & 648 \\
Number & 8 & 1 & 6 & 2 & 2 & 1 & 3 & 6 & 1 \\
\hline Degree & 702 & 864 & 936 & 1404 & 1458 & 2106 & 4212 & 5616 & 6318 \\
Number & 13 & 4 & 3 & 16 & 1 & 11 & 6 & 12 & 2 \\
\hline
\end{tabular}

Table A.19: The degrees of characters in $\operatorname{Irr}\left(3^{1+8} \cdot 3^{1+2} \cdot\left(2 S_{4} \times 2\right)\right)$.

\begin{tabular}{l|rrrrrrrrrrrrrr}
\hline Degree & 1 & 2 & 3 & 4 & 6 & 8 & 12 & 16 & 18 & 32 & 36 & 48 & 54 & 72 \\
Number & 4 & 6 & 4 & 2 & 6 & 8 & 6 & 6 & 4 & 1 & 6 & 8 & 4 & 16 \\
\hline Degree & 108 & 144 & 162 & 216 & 288 & 324 & 432 & 486 & 648 & 864 & 972 & 1296 & 1458 \\
Number & 4 & 12 & 8 & 25 & 3 & 6 & 14 & 14 & 12 & 1 & 12 & 10 & 4 \\
\hline
\end{tabular}


Table A.20: The degrees of characters in $\operatorname{Irr}\left(2 \times 3^{1+8} \cdot 3^{1+2} \cdot\left(2 S_{4} \times 2\right) \mid \xi\right)$.

\begin{tabular}{l|rrrrrrrrrrrrrr}
\hline Degree & 1 & 2 & 3 & 4 & 6 & 8 & 12 & 16 & 18 & 32 & 36 & 48 & 54 & 72 \\
Number & 4 & 6 & 4 & 2 & 6 & 8 & 6 & 6 & 4 & 1 & 6 & 8 & 4 & 16 \\
\hline Degree & 108 & 144 & 162 & 216 & 288 & 324 & 432 & 486 & 648 & 864 & 972 & 1296 & 1458 & \\
Number & 4 & 12 & 8 & 25 & 3 & 6 & 14 & 14 & 12 & 1 & 12 & 10 & 4 \\
\hline
\end{tabular}

Table A.21: The degrees of characters in $\operatorname{Irr}\left(3^{1+8} \cdot 3^{1+2} \cdot 3 \cdot 2^{3}\right)$.

\begin{tabular}{l|rrrrrrrrr}
\hline Degree & 1 & 2 & 4 & 6 & 8 & 12 & 18 & 24 & 36 \\
Number & 8 & 12 & 6 & 20 & 1 & 12 & 16 & 1 & 22 \\
\hline Degree & 54 & 72 & 108 & 162 & 216 & 324 & 486 & 648 & 972 \\
Number & 52 & 3 & 34 & 48 & 4 & 26 & 24 & 1 & 2 \\
\hline
\end{tabular}

Table A.22: The degrees of characters in $\operatorname{Irr}\left(2 \times 3^{1+8} \cdot 3^{1+2} \cdot 3 \cdot 2^{3} \mid \xi\right)$.

\begin{tabular}{l|rrrrrrrrr}
\hline Degree & 1 & 2 & 4 & 6 & 8 & 12 & 18 & 24 & 36 \\
Number & 8 & 12 & 6 & 20 & 1 & 12 & 16 & 1 & 22 \\
\hline Degree & 54 & 72 & 108 & 162 & 216 & 324 & 486 & 648 & 972 \\
Number & 52 & 3 & 34 & 48 & 4 & 26 & 24 & 1 & 2 \\
\hline
\end{tabular}

Table A.23: The degrees of characters in $\operatorname{Irr}\left(S_{3} \times\left(S_{3} \times U_{4}(3): 2\right) .2\right)$.

\begin{tabular}{l|rrrrrrrrrrrr}
\hline Degree & 1 & 2 & 4 & 21 & 35 & 42 & 70 & 84 & 90 & 140 & 180 & 189 \\
Number & 16 & 16 & 4 & 16 & 32 & 16 & 32 & 4 & 16 & 24 & 16 & 16 \\
\hline Degree & 210 & 280 & 315 & 360 & 378 & 420 & 560 & 630 & 729 & 756 & 840 & 896 \\
Number & 16 & 16 & 32 & 4 & 16 & 32 & 20 & 32 & 16 & 4 & 20 & 16 \\
\hline Degree & 1120 & 1260 & 1280 & 1458 & 1680 & 1792 & 2240 & 2560 & 2916 & 3584 & 4480 & 5120 \\
Number & 20 & 8 & 8 & 16 & 4 & 16 & 8 & 8 & 4 & 4 & 1 & 2 \\
\hline
\end{tabular}


Table A.24: The degrees of characters in $\operatorname{Irr}\left(2 .\left(S_{3} \times\left(S_{3} \times U_{4}(3): 2\right) .2 \mid \xi\right)\right.$.

\begin{tabular}{l|rrrrrrrrrr}
\hline Degree & 40 & 80 & 112 & 140 & 224 & 240 & 280 & 448 & 480 & 560 \\
Number & 4 & 8 & 8 & 12 & 8 & 4 & 14 & 2 & 8 & 7 \\
\hline Degree & 840 & 1008 & 1080 & 1120 & 1260 & 1680 & 1792 & 2016 & 2160 & 2240 \\
Number & 2 & 8 & 4 & 4 & 4 & 4 & 4 & 8 & 8 & 4 \\
\hline Degree & 2520 & 2560 & 3584 & 4032 & 4480 & 5040 & 5120 & 7168 & & \\
Number & 4 & 2 & 4 & 2 & 1 & 1 & 4 & 1 & & \\
\hline
\end{tabular}

Table A.25: The degrees of characters in $\operatorname{Irr}\left(S_{3} \times S_{3} \times 3^{1+4} 2_{-}^{1+4} \cdot S_{3}\right)$.

\begin{tabular}{l|rrrrrrrrrrr}
\hline Degree & 1 & 2 & 3 & 4 & 6 & 8 & 12 & 16 & 18 & 32 & 36 \\
Number & 32 & 48 & 32 & 32 & 32 & 16 & 8 & 38 & 48 & 49 & 48 \\
\hline Degree & 48 & 54 & 64 & 72 & 96 & 108 & 128 & 144 & 192 & 216 & 288 \\
Number & 16 & 16 & 24 & 24 & 16 & 16 & 4 & 12 & 4 & 4 & 3 \\
\hline
\end{tabular}

Table A.26: The degrees of characters in $\operatorname{Irr}\left(2 .\left(S_{3} \times S_{3} \times 3^{1+4} 2_{-}^{1+4} \cdot S_{3}\right) \mid \xi\right)$.

\begin{tabular}{l|rrrrrrrr}
\hline Degree & 4 & 8 & 12 & 16 & 24 & 32 & 36 & 64 \\
Number & 14 & 24 & 2 & 6 & 4 & 17 & 12 & 18 \\
\hline Degree & 72 & 96 & 108 & 128 & 144 & 192 & 216 & 288 \\
Number & 36 & 4 & 4 & 8 & 12 & 8 & 8 & 3 \\
\hline
\end{tabular}

Table A.27: The degrees of characters in $\operatorname{Irr}\left(S_{3} \times 3^{2} \cdot 3^{2} \cdot 3^{3} \cdot 2^{3} \cdot 2^{2}\right)$.

\begin{tabular}{l|rrrrrrrrrrrrrr}
\hline Degree & 1 & 2 & 4 & 8 & 12 & 16 & 18 & 24 & 32 & 36 & 48 & 72 & 96 & 144 \\
Number & 32 & 56 & 68 & 58 & 32 & 25 & 48 & 48 & 4 & 60 & 24 & 24 & 4 & 3 \\
\hline
\end{tabular}

Table A.28: The degrees of characters in $\operatorname{Irr}\left(2 .\left(S_{3} \times 3^{2} \cdot 3^{2} \cdot 3^{3} \cdot 2^{3} \cdot 2^{2}\right) \mid \xi\right)$.

\begin{tabular}{l|rrrrrrrrrrr}
\hline Degree & 2 & 4 & 8 & 16 & 24 & 32 & 36 & 48 & 72 & 96 & 144 \\
Number & 8 & 18 & 34 & 19 & 16 & 8 & 24 & 18 & 36 & 8 & 3 \\
\hline
\end{tabular}


Table A.29: The degrees of characters in $\operatorname{Irr}\left(S_{3} \times S_{3} \times 3^{4}: 2 S_{6}\right)$.

\begin{tabular}{l|rrrrrrrrrrrr}
\hline Degree & 1 & 2 & 4 & 5 & 9 & 10 & 16 & 18 & 20 & 30 & 32 & 36 \\
Number & 16 & 16 & 4 & 32 & 16 & 48 & 8 & 16 & 40 & 32 & 8 & 4 \\
\hline Degree & 40 & 60 & 64 & 80 & 90 & 120 & 160 & 180 & 240 & 320 & 360 & \\
Number & 24 & 48 & 2 & 24 & 32 & 24 & 17 & 32 & 4 & 4 & 8 & \\
\hline
\end{tabular}

Table A.30: The degrees of characters in $\operatorname{Irr}\left(2 .\left(S_{3} \times S_{3} \times 3^{4}: 2 S_{6}\right) \mid \xi\right)$.

\begin{tabular}{l|rrrrrrrrrr}
\hline Degree & 2 & 4 & 8 & 10 & 18 & 20 & 32 & 36 & 40 & 60 \\
Number & 4 & 4 & 1 & 8 & 4 & 12 & 2 & 4 & 14 & 16 \\
\hline Degree & 64 & 80 & 120 & 160 & 180 & 240 & 320 & 360 & 720 & \\
Number & 4 & 1 & 18 & 11 & 8 & 8 & 6 & 8 & 2 & \\
\hline
\end{tabular}

Table A.31: The degrees of characters in $\operatorname{Irr}\left(S_{3} \times 3^{5}:\left(2 \times U_{4}(2): 2\right)\right)$.

\begin{tabular}{l|rrrrrrrrrrr}
\hline Degree & 1 & 2 & 6 & 10 & 12 & 15 & 20 & 24 & 30 & 40 & 48 \\
Number & 8 & 4 & 8 & 4 & 4 & 16 & 14 & 8 & 16 & 6 & 4 \\
\hline Degree & 60 & 64 & 72 & 80 & 81 & 90 & 120 & 128 & 144 & 160 & 162 \\
Number & 16 & 8 & 8 & 12 & 8 & 12 & 6 & 4 & 4 & 10 & 4 \\
\hline Degree & 180 & 240 & 320 & 360 & 480 & 540 & 640 & 648 & 720 & 810 & 960 \\
Number & 14 & 8 & 2 & 30 & 12 & 4 & 4 & 8 & 29 & 8 & 14 \\
\hline Degree & 1080 & 1152 & 1280 & 1296 & 1440 & 1620 & 1920 & 2160 & 2304 & 2560 & 2880 \\
Number & 4 & 4 & 4 & 4 & 10 & 4 & 5 & 1 & 2 & 1 & 1 \\
\hline
\end{tabular}

Table A.32: The degrees of characters in $\operatorname{Irr}\left(2 .\left(S_{3} \times 3^{5}:\left(2 \times U_{4}(2): 2\right)\right) \mid \xi\right)$.

\begin{tabular}{l|rrrrrrrrrr}
\hline Degree & 2 & 4 & 12 & 20 & 24 & 30 & 40 & 48 & 60 & 80 \\
Number & 2 & 1 & 2 & 3 & 1 & 4 & 5 & 2 & 4 & 1 \\
\hline Degree & 96 & 120 & 128 & 144 & 160 & 162 & 180 & 240 & 256 & 288 \\
Number & 1 & 6 & 2 & 2 & 9 & 2 & 9 & 1 & 1 & 1 \\
\hline Degree & 320 & 324 & 360 & 480 & 720 & 960 & 1080 & 1280 & 1296 & 1440 \\
Number & 3 & 1 & 8 & 2 & 11 & 9 & 5 & 5 & 2 & 12 \\
\hline Degree & 1620 & 1920 & 2160 & 2304 & 2560 & 2592 & 2880 & 3240 & 3840 & \\
Number & 2 & 3 & 1 & 3 & 1 & 1 & 2 & 1 & 1 & \\
\hline
\end{tabular}


Table A.33: The degrees of characters in $\operatorname{Irr}\left(S_{3} \times \mathrm{Fi}_{22}: 2\right)$.

\begin{tabular}{|c|c|c|c|c|c|c|c|}
\hline Degree & 1 & 2 & 78 & 156 & 429 & 858 & 1001 \\
\hline Number & 4 & 2 & 4 & 2 & 4 & 2 & 4 \\
\hline Degree & 1430 & 2002 & 2860 & 3003 & 3080 & 6006 & 6160 \\
\hline Number & 4 & 2 & 2 & 4 & 4 & 2 & 2 \\
\hline Degree & 10725 & 13650 & 21450 & 27300 & 30030 & 32032 & 43680 \\
\hline Number & 4 & 4 & 2 & 2 & 4 & 4 & 4 \\
\hline Degree & 45045 & 48048 & 50050 & 60060 & 64064 & 75075 & 81081 \\
\hline Number & 4 & 4 & 8 & 2 & 2 & 12 & 4 \\
\hline Degree & 87360 & 90090 & 96096 & 100100 & 114400 & 150150 & 162162 \\
\hline Number & 2 & 2 & 2 & 4 & 4 & 10 & 2 \\
\hline Degree & 205920 & 228800 & 277200 & 289575 & 300300 & 320320 & 360855 \\
\hline Number & 4 & 2 & 2 & 4 & 6 & 4 & 4 \\
\hline Degree & 370656 & 411840 & 450450 & 554400 & 576576 & 577368 & 579150 \\
\hline Number & 4 & 2 & 8 & 1 & 4 & 4 & 6 \\
\hline Degree & 600600 & 640640 & 675675 & 720720 & 721710 & 741312 & 800800 \\
\hline Number & 6 & 2 & 4 & 4 & 2 & 2 & 8 \\
\hline Degree & 852930 & 900900 & 938223 & 972972 & 1153152 & 1154736 & 1158300 \\
\hline Number & 4 & 4 & 4 & 4 & 2 & 2 & 2 \\
\hline Degree & 1164800 & 1201200 & 1351350 & 1360800 & 1372800 & 1441440 & 1441792 \\
\hline Number & 2 & 8 & 2 & 4 & 4 & 2 & 4 \\
\hline Degree & 1601600 & 1705860 & 1791153 & 1876446 & 1945944 & 1965600 & 2027025 \\
\hline Number & 4 & 2 & 4 & 6 & 2 & 2 & 4 \\
\hline Degree & 2050048 & 2316600 & 2329600 & 2402400 & 2555904 & 2721600 & 2729376 \\
\hline Number & 4 & 4 & 1 & 7 & 8 & 2 & 4 \\
\hline Degree & 2745600 & 2883584 & 3582306 & 3752892 & 3931200 & 4054050 & 4100096 \\
\hline Number & 2 & 2 & 2 & 2 & 1 & 2 & 2 \\
\hline Degree & 4633200 & 4804800 & 5111808 & 5458752 & & & \\
\hline Number & 2 & 2 & 4 & 2 & & & \\
\hline
\end{tabular}


Table A.34: The degrees of characters in $\left.\operatorname{Irr}\left(\left(S_{3} \times 2 . \mathrm{Fi}_{22}\right): 2\right) \mid \xi\right)$.

\begin{tabular}{l|rrrrrrr}
\hline Degree & 704 & 4160 & 8320 & 11648 & 23296 & 27456 & 54912 \\
Number & 3 & 2 & 1 & 4 & 2 & 2 & 4 \\
\hline Degree & 96096 & 192192 & 211200 & 246400 & 266112 & 292864 & 457600 \\
Number & 2 & 1 & 6 & 3 & 3 & 2 & 3 \\
\hline Degree & 471744 & 585728 & 640640 & 800800 & 873600 & 943488 & 960960 \\
Number & 2 & 1 & 2 & 2 & 2 & 1 & 2 \\
\hline Degree & 1281280 & 1372800 & 1601600 & 1747200 & 1830400 & 1921920 & 2059200 \\
Number & 1 & 2 & 1 & 1 & 2 & 1 & 3 \\
\hline Degree & 2402400 & 2594592 & 2745600 & 2883584 & 3326400 & 3660800 & 4392960 \\
Number & 2 & 2 & 1 & 3 & 2 & 1 & 2 \\
\hline Degree & 4717440 & 4804800 & 5111808 & 5189184 & 6652800 & 8785920 & 9434880 \\
Number & 2 & 3 & 2 & 1 & 1 & 1 & 1 \\
\hline Degree & 9609600 & 10223616 & & & & & \\
Number & 1 & 1 & & & & & \\
\hline
\end{tabular}

Table A.35: The degrees of characters in $\operatorname{Irr}\left(S_{3} \times 3^{3+3}: L_{3}(3)\right)$.

\begin{tabular}{l|rrrrrrrrr}
\hline Degree & 1 & 2 & 12 & 13 & 16 & 24 & 26 & 27 & 32 \\
Number & 2 & 1 & 2 & 2 & 8 & 1 & 13 & 2 & 4 \\
\hline Degree & 39 & 52 & 54 & 78 & 104 & 156 & 208 & 234 & 312 \\
Number & 2 & 12 & 1 & 9 & 3 & 10 & 6 & 14 & 3 \\
\hline Degree & 416 & 468 & 624 & 702 & 936 & 1248 & 1404 & & \\
Number & 3 & 19 & 6 & 4 & 6 & 3 & 2 & & \\
\hline
\end{tabular}

Table A.36: The degrees of characters in $\operatorname{Irr}\left(2 \times S_{3} \times 3^{3+3}: L_{3}(3) \mid \xi\right)$.

\begin{tabular}{l|rrrrrrrrr}
\hline Degree & 1 & 2 & 12 & 13 & 16 & 24 & 26 & 27 & 32 \\
Number & 2 & 1 & 2 & 2 & 8 & 1 & 13 & 2 & 4 \\
\hline Degree & 39 & 52 & 54 & 78 & 104 & 156 & 208 & 234 & 312 \\
Number & 2 & 12 & 1 & 9 & 3 & 10 & 6 & 14 & 3 \\
\hline Degree & 416 & 468 & 624 & 702 & 936 & 1248 & 1404 & & \\
Number & 3 & 19 & 6 & 4 & 6 & 3 & 2 & & \\
\hline
\end{tabular}


Table A.37: The degrees of characters in $\operatorname{Irr}\left(S_{3} \times 3^{5} \cdot 3^{1+2} \cdot 2 S_{4}\right)$.

\begin{tabular}{l|rrrrrrrrrr}
\hline Degree & 1 & 2 & 3 & 4 & 6 & 8 & 12 & 16 & 18 & 24 \\
Number & 4 & 8 & 4 & 5 & 8 & 17 & 9 & 16 & 20 & 3 \\
\hline Degree & 32 & 36 & 48 & 54 & 72 & 96 & 108 & 144 & 288 & \\
Number & 4 & 28 & 8 & 6 & 45 & 4 & 3 & 36 & 9 & \\
\hline
\end{tabular}

Table A.38: The degrees of characters in $\operatorname{Irr}\left(2 .\left(S_{3} \times 3^{5} \cdot 3^{1+2} \cdot 2 S_{4}\right) \mid \xi\right)$.

\begin{tabular}{l|rrrrrrrrrr}
\hline Degree & 1 & 2 & 3 & 4 & 6 & 8 & 12 & 16 & 18 & 24 \\
Number & 4 & 8 & 4 & 5 & 8 & 17 & 9 & 16 & 20 & 3 \\
\hline Degree & 32 & 36 & 48 & 54 & 72 & 96 & 108 & 144 & 288 & \\
Number & 4 & 28 & 8 & 6 & 45 & 4 & 3 & 36 & 9 & \\
\hline
\end{tabular}

Table A.39: The degrees of characters in $\operatorname{Irr}\left(S_{3} \times 3^{1+6} 2^{3+4}: 3^{2} \cdot 2^{2}\right)$.

\begin{tabular}{l|rrrrrrrrr}
\hline Degree & 1 & 2 & 4 & 6 & 8 & 9 & 12 & 16 & 18 \\
Number & 8 & 12 & 14 & 12 & 13 & 8 & 20 & 6 & 4 \\
\hline Degree & 24 & 32 & 36 & 48 & 54 & 72 & 96 & 108 & 128 \\
Number & 27 & 1 & 2 & 18 & 12 & 17 & 8 & 12 & 8 \\
\hline Degree & 144 & 192 & 216 & 256 & 324 & 384 & 432 & 486 & 512 \\
Number & 8 & 20 & 15 & 12 & 18 & 25 & 12 & 4 & 6 \\
\hline Degree & 648 & 768 & 864 & 972 & 1024 & 1296 & 1536 & 1944 & \\
Number & 21 & 12 & 3 & 6 & 1 & 6 & 2 & 2 & \\
\hline
\end{tabular}

Table A.40: The degrees of characters in $\operatorname{Irr}\left(2 .\left(S_{3} \times 3^{1+6} 2^{3+4}: 3^{2} \cdot 2^{2}\right) \mid \xi\right)$.

\begin{tabular}{l|rrrrrrrrrr}
\hline Degree & 4 & 8 & 12 & 16 & 24 & 48 & 96 & 192 & 216 & 256 \\
Number & 4 & 10 & 4 & 4 & 8 & 5 & 14 & 4 & 18 & 6 \\
\hline Degree & 288 & 384 & 432 & 512 & 648 & 768 & 1024 & 1296 & 1536 & 2592 \\
Number & 3 & 5 & 9 & 8 & 6 & 6 & 1 & 9 & 5 & 3 \\
\hline
\end{tabular}

Table A.41: The degrees of characters in $\operatorname{Irr}\left(S_{3} \times 3^{1+6} \cdot 3: 2 S_{4}\right)$.

\begin{tabular}{l|rrrrrrrrrr}
\hline Degree & 1 & 2 & 3 & 4 & 6 & 8 & 12 & 16 & 24 & 32 \\
Number & 4 & 14 & 4 & 14 & 4 & 8 & 1 & 10 & 24 & 4 \\
\hline Degree & 48 & 54 & 72 & 96 & 108 & 144 & 162 & 216 & 288 & 324 \\
Number & 24 & 18 & 24 & 6 & 27 & 14 & 6 & 9 & 1 & 3 \\
\hline
\end{tabular}


Table A.42: The degrees of characters in $\operatorname{Irr}\left(2 .\left(S_{3} \times 3^{1+6} \cdot 3: 2 S_{4}\right) \mid \xi\right)$.

\begin{tabular}{l|rrrrrrrrrr}
\hline Degree & 1 & 2 & 3 & 4 & 6 & 8 & 12 & 16 & 24 & 32 \\
Number & 4 & 14 & 4 & 14 & 4 & 8 & 1 & 10 & 24 & 4 \\
\hline Degree & 48 & 54 & 72 & 96 & 108 & 144 & 162 & 216 & 288 & 324 \\
Number & 24 & 18 & 24 & 6 & 27 & 14 & 6 & 9 & 1 & 3 \\
\hline
\end{tabular}

Table A.43: The degrees of characters in $\operatorname{Irr}\left(S_{3} \times 3^{1+6} \cdot 3^{2} \cdot 2^{2}\right)$.

\begin{tabular}{l|rrrrrrrrrrrr}
\hline Degree & 1 & 2 & 4 & 6 & 8 & 12 & 18 & 24 & 36 & 54 & 72 & 108 \\
Number & 8 & 24 & 18 & 28 & 4 & 28 & 72 & 7 & 56 & 36 & 10 & 18 \\
\hline
\end{tabular}

Table A.44: The degrees of characters in $\operatorname{Irr}\left(2 \cdot\left(S_{3} \times 3^{1+6} \cdot 3^{2} \cdot 2^{2}\right) \mid \xi\right)$.

\begin{tabular}{l|rrrrrrrrrrrr}
\hline Degree & 1 & 2 & 4 & 6 & 8 & 12 & 18 & 24 & 36 & 54 & 72 & 108 \\
Number & 8 & 24 & 18 & 28 & 4 & 28 & 72 & 7 & 56 & 36 & 10 & 18 \\
\hline
\end{tabular}

Table A.45: The degrees of characters in $\operatorname{Irr}\left(S_{3} \times 3^{5} \cdot 3^{3} \cdot\left(2 \times S_{4}\right) .2\right)$.

\begin{tabular}{l|rrrrrrrr}
\hline Degree & 1 & 2 & 3 & 4 & 6 & 8 & 12 & 16 \\
Number & 16 & 24 & 16 & 12 & 32 & 10 & 40 & 12 \\
\hline Degree & 18 & 24 & 32 & 36 & 48 & 54 & 64 & 72 \\
Number & 16 & 32 & 6 & 28 & 17 & 24 & 1 & 42 \\
\hline Degree & 96 & 108 & 144 & 162 & 192 & 216 & 288 & 324 \\
Number & 6 & 24 & 24 & 24 & 1 & 6 & 4 & 12 \\
\hline
\end{tabular}

Table A.46: The degrees of characters in $\operatorname{Irr}\left(2 .\left(S_{3} \times 3^{5} \cdot 3^{3} \cdot\left(2 \times S_{4}\right) .2\right) \mid \xi\right)$.

\begin{tabular}{l|rrrrrrrrrr}
\hline Degree & 2 & 4 & 6 & 8 & 12 & 16 & 24 & 32 & 36 & 48 \\
Number & 4 & 10 & 4 & 4 & 8 & 6 & 18 & 8 & 4 & 11 \\
\hline Degree & 64 & 72 & 96 & 108 & 144 & 192 & 216 & 288 & 324 & 648 \\
Number & 1 & 17 & 9 & 18 & 20 & 1 & 9 & 7 & 6 & 3 \\
\hline
\end{tabular}


Table A.47: The degrees of characters in $\operatorname{Irr}\left(\left(3^{2}: D_{8} \times 3^{4}: A_{6}: 2^{2}\right) .2\right)$.

\begin{tabular}{l|rrrrrrrrr}
\hline Degree & 1 & 2 & 4 & 8 & 9 & 10 & 16 & 18 & 20 \\
Number & 8 & 10 & 1 & 8 & 8 & 24 & 8 & 10 & 22 \\
\hline Degree & 32 & 36 & 40 & 60 & 72 & 80 & 120 & 128 & 160 \\
Number & 6 & 1 & 31 & 16 & 8 & 12 & 12 & 4 & 16 \\
\hline Degree & 180 & 240 & 320 & 360 & 480 & 640 & 720 & & \\
Number & 16 & 18 & 4 & 4 & 8 & 8 & 16 & & \\
\hline
\end{tabular}

Table A.48: The degrees of characters in $\operatorname{Irr}\left(2 .\left(\left(3^{2}: D_{8} \times 3^{4}: A_{6}: 2^{2}\right) .2\right) \mid \xi\right)$.

\begin{tabular}{l|rrrrrrrrrrrr}
\hline Degree & 2 & 4 & 16 & 18 & 20 & 32 & 36 & 40 & 64 & 80 & 120 & 144 \\
Number & 4 & 3 & 2 & 4 & 8 & 4 & 3 & 8 & 1 & 11 & 4 & 2 \\
\hline Degree & 160 & 240 & 256 & 320 & 360 & 480 & 640 & 720 & 960 & 1280 & 1440 & \\
Number & 2 & 5 & 1 & 8 & 4 & 8 & 4 & 1 & 1 & 1 & 4 & \\
\hline
\end{tabular}

Table A.49: The degrees of characters in $\operatorname{Irr}\left(3^{3} \cdot 3^{2} \cdot 3^{3} \cdot 2^{3} \cdot 2^{2} \cdot 2^{3}\right)$.

\begin{tabular}{l|rrrrrrrr}
\hline Degree & 1 & 2 & 4 & 8 & 16 & 18 & 24 & 32 \\
Number & 16 & 36 & 22 & 36 & 24 & 8 & 16 & 20 \\
\hline Degree & 36 & 48 & 64 & 72 & 96 & 144 & 192 & 288 \\
Number & 30 & 12 & 8 & 12 & 18 & 25 & 8 & 6 \\
\hline
\end{tabular}

Table A.50: The degrees of characters in $\operatorname{Irr}\left(2 \cdot\left(3^{3} \cdot 3^{2} \cdot 3^{3} \cdot 2^{3} \cdot 2^{2} \cdot 2^{3}\right) \mid \xi\right)$.

\begin{tabular}{l|rrrrrrrrrrrrrrr}
\hline Degree & 2 & 4 & 8 & 16 & 32 & 36 & 48 & 64 & 72 & 96 & 128 & 144 & 192 & 288 & 384 \\
Number & 8 & 14 & 8 & 8 & 10 & 4 & 4 & 8 & 11 & 5 & 1 & 3 & 8 & 12 & 1 \\
\hline
\end{tabular}

Table A.51: The degrees of characters in $\operatorname{Irr}\left(\left(3^{2}: D_{8} \times 3^{1+4}: 2 S_{4}: 2^{2}.\right) .2\right)$.

\begin{tabular}{l|rrrrrrrrr}
\hline Degree & 1 & 2 & 3 & 4 & 6 & 8 & 12 & 16 & 18 \\
Number & 16 & 28 & 16 & 20 & 20 & 27 & 2 & 11 & 8 \\
\hline Degree & 24 & 32 & 36 & 48 & 54 & 64 & 72 & 96 & 108 \\
Number & 16 & 20 & 26 & 8 & 8 & 14 & 9 & 10 & 10 \\
\hline Degree & 128 & 144 & 192 & 216 & 256 & 288 & 384 & 432 & 576 \\
Number & 18 & 31 & 1 & 1 & 8 & 1 & 8 & 8 & 6 \\
\hline
\end{tabular}


Table A.52: The degrees of characters in $\operatorname{Irr}\left(2 .\left(\left(3^{2}: D_{8} \times 3^{1+4}: 2 S_{4}: 2^{2}.\right) .2\right) \mid \xi\right)$.

\begin{tabular}{l|rrrrrrrrrr}
\hline Degree & 4 & 8 & 12 & 16 & 24 & 32 & 64 & 72 & 96 & 128 \\
Number & 12 & 15 & 4 & 3 & 1 & 7 & 6 & 8 & 1 & 5 \\
\hline Degree & 144 & 192 & 216 & 256 & 288 & 512 & 576 & 768 & 864 & \\
Number & 9 & 4 & 4 & 8 & 7 & 1 & 6 & 2 & 2 & \\
\hline
\end{tabular}

Table A.53: The degrees of characters in $\operatorname{Irr}\left(\left(3^{2}: D_{8} \times U_{4}(3): 2^{2}.\right) .2\right)$.

\begin{tabular}{l|rrrrrrrrr}
\hline Degree & 1 & 2 & 4 & 8 & 21 & 42 & 70 & 84 & 90 \\
Number & 8 & 10 & 1 & 8 & 8 & 10 & 16 & 1 & 8 \\
\hline Degree & 140 & 168 & 180 & 189 & 210 & 280 & 360 & 378 & 420 \\
Number & 12 & 8 & 10 & 8 & 8 & 26 & 1 & 10 & 18 \\
\hline Degree & 560 & 630 & 720 & 729 & 756 & 840 & 896 & 1120 & 1260 \\
Number & 1 & 16 & 8 & 8 & 1 & 11 & 8 & 20 & 4 \\
\hline Degree & 1280 & 1458 & 1512 & 1680 & 1792 & 2240 & 2520 & 2560 & 2916 \\
Number & 8 & 10 & 8 & 9 & 10 & 5 & 16 & 6 & 1 \\
\hline Degree & 3360 & 3584 & 4480 & 5832 & 7168 & 8960 & 10240 & & \\
Number & 8 & 1 & 8 & 8 & 8 & 2 & 4 & & \\
\hline
\end{tabular}

Table A.54: The degrees of characters in $\operatorname{Irr}\left(2 .\left(\left(3^{2}: D_{8} \times U_{4}(3): 2^{2}.\right) .2\right) \mid \xi\right)$.

\begin{tabular}{l|rrrrrrrrrr}
\hline Degree & 80 & 140 & 224 & 280 & 320 & 448 & 480 & 560 & 840 & 896 \\
Number & 4 & 4 & 4 & 3 & 2 & 1 & 4 & 4 & 4 & 4 \\
\hline Degree & 1120 & 1260 & 1680 & 1792 & 1920 & 2016 & 2160 & 2240 & 2520 & 2560 \\
Number & 10 & 4 & 1 & 4 & 2 & 4 & 4 & 4 & 3 & 4 \\
\hline Degree & 3584 & 4032 & 5120 & 6720 & 8064 & 8640 & 8960 & 10080 & 14336 & 20480 \\
Number & 3 & 1 & 1 & 1 & 4 & 2 & 2 & 2 & 2 & 1 \\
\hline
\end{tabular}

Table A.55: The degrees of characters in $\operatorname{Irr}\left(3^{3+6} \cdot\left(2^{2} \times L_{3}(3)\right)\right)$.

\begin{tabular}{l|rrrrrrrrrr}
\hline Degree & 1 & 12 & 13 & 16 & 26 & 27 & 39 & 52 & 78 & 104 \\
Number & 4 & 4 & 4 & 16 & 20 & 4 & 4 & 14 & 8 & 7 \\
\hline Degree & 156 & 208 & 234 & 416 & 468 & 624 & 702 & 832 & 936 & 1248 \\
Number & 2 & 9 & 4 & 6 & 10 & 4 & 4 & 1 & 8 & 4 \\
\hline Degree & 1404 & 1872 & 2496 & 2808 & 3744 & 5616 & 7488 & 8424 & & \\
Number & 4 & 6 & 1 & 3 & 6 & 3 & 2 & 1 & & \\
\hline
\end{tabular}


Table A.56: The degrees of characters in $\operatorname{Irr}\left(2.3^{3+6} \cdot\left(2^{2} \times L_{3}(3)\right) \mid \xi\right)$.

\begin{tabular}{l|rrrrrrrrrrrr}
\hline Degree & 2 & 24 & 26 & 32 & 52 & 54 & 78 & 104 & 156 & 208 & 416 & 468 \\
Number & 1 & 1 & 1 & 4 & 11 & 1 & 1 & 9 & 4 & 1 & 8 & 7 \\
\hline Degree & 832 & 936 & 1248 & 1404 & 1872 & 2496 & 2808 & 3744 & 5616 & 7488 & 8424 & \\
Number & 1 & 9 & 5 & 5 & 2 & 1 & 3 & 7 & 3 & 2 & 1 & \\
\hline
\end{tabular}

Table A.57: The degrees of characters in $\operatorname{Irr}\left(3^{2} \cdot 3^{3} \cdot 3^{6} \cdot\left(2^{2} \times 2 S_{4}\right)\right)$.

\begin{tabular}{l|rrrrrrrrrrrr}
\hline Degree & 1 & 2 & 3 & 4 & 8 & 16 & 18 & 32 & 36 & 48 & 54 & 64 \\
Number & 8 & 12 & 8 & 4 & 24 & 24 & 12 & 8 & 24 & 12 & 12 & 1 \\
\hline Degree & 72 & 96 & 108 & 144 & 192 & 216 & 288 & 432 & 576 & 648 & 864 & 1728 \\
Number & 15 & 6 & 6 & 15 & 1 & 11 & 12 & 19 & 3 & 1 & 16 & 5 \\
\hline
\end{tabular}

Table A.58: The degrees of characters in $\left.\operatorname{Irr}\left(2 \cdot 3^{2} \cdot 3^{3} \cdot 3^{6} \cdot\left(2^{2} \times 2 S_{4}\right)\right) \mid \xi\right)$.

\begin{tabular}{l|rrrrrrrrrr}
\hline Degree & 2 & 4 & 6 & 8 & 16 & 32 & 36 & 64 & 72 & 96 \\
Number & 2 & 3 & 2 & 1 & 18 & 11 & 15 & 1 & 18 & 9 \\
\hline Degree & 108 & 144 & 192 & 216 & 288 & 432 & 576 & 648 & 864 & 1728 \\
Number & 9 & 3 & 1 & 3 & 15 & 17 & 3 & 1 & 17 & 5 \\
\hline
\end{tabular}

Table A.59: The degrees of characters in $\operatorname{Irr}\left(3^{3+6} \cdot 3^{1+2} \cdot 2^{4}\right)$.

\begin{tabular}{l|rrrrrrrrrr}
\hline Degree & 1 & 2 & 4 & 6 & 8 & 12 & 16 & 18 & 24 & 36 \\
Number & 16 & 32 & 24 & 24 & 8 & 36 & 1 & 24 & 20 & 36 \\
\hline Degree & 48 & 54 & 72 & 108 & 144 & 162 & 216 & 324 & 432 & 648 \\
Number & 7 & 8 & 18 & 28 & 3 & 8 & 28 & 12 & 5 & 10 \\
\hline
\end{tabular}

Table A.60: The degrees of characters in $\left.\operatorname{Irr}\left(2.3^{3+6} \cdot 3^{1+2} \cdot 2^{4}\right) \mid \xi\right)$.

\begin{tabular}{l|rrrrrrrrrrrrrrr}
\hline Degree & 2 & 4 & 8 & 12 & 16 & 24 & 36 & 48 & 72 & 108 & 144 & 216 & 324 & 432 & 648 \\
Number & 4 & 20 & 11 & 18 & 1 & 26 & 30 & 7 & 21 & 14 & 3 & 32 & 14 & 5 & 10 \\
\hline
\end{tabular}


Table A.61: The degrees of characters in $\operatorname{Irr}\left(3^{6} \cdot 3^{2+3} \cdot\left(2^{2} \times 2 S_{4}\right)\right)$.

\begin{tabular}{l|rrrrrrrrr}
\hline Degree & 1 & 2 & 3 & 4 & 6 & 8 & 12 & 16 & 24 \\
Number & 8 & 20 & 8 & 18 & 8 & 15 & 2 & 13 & 16 \\
\hline Degree & 32 & 48 & 64 & 72 & 96 & 144 & 162 & 192 & 288 \\
Number & 6 & 28 & 1 & 8 & 18 & 16 & 4 & 7 & 10 \\
\hline Degree & 324 & 432 & 486 & 576 & 648 & 864 & 972 & 1296 & 1944 \\
Number & 10 & 8 & 4 & 2 & 11 & 10 & 4 & 5 & 1 \\
\hline
\end{tabular}

Table A.62: The degrees of characters in $\left.\operatorname{Irr}\left(2.3^{6} \cdot 3^{2+3} \cdot\left(2^{2} \times 2 S_{4}\right)\right) \mid \xi\right)$.

\begin{tabular}{l|rrrrrrrrrr}
\hline Degree & 2 & 4 & 6 & 8 & 12 & 16 & 32 & 48 & 64 & 96 \\
Number & 2 & 11 & 2 & 10 & 4 & 3 & 9 & 16 & 1 & 22 \\
\hline Degree & 144 & 192 & 288 & 324 & 576 & 648 & 864 & 972 & 1296 & 1944 \\
Number & 14 & 7 & 11 & 7 & 2 & 12 & 12 & 5 & 5 & 1 \\
\hline
\end{tabular}

Table A.63: The degrees of characters in $\operatorname{Irr}\left(3^{1+8} \cdot 3 \cdot 2^{2} \cdot 2^{4} \cdot 3^{2} \cdot D_{8}\right)$.

\begin{tabular}{l|rrrrrrrrrr}
\hline Degree & 1 & 2 & 4 & 6 & 8 & 9 & 12 & 16 & 18 & 24 \\
Number & 8 & 6 & 17 & 8 & 10 & 8 & 8 & 9 & 6 & 2 \\
\hline Degree & 32 & 36 & 48 & 64 & 96 & 128 & 144 & 162 & 192 & 256 \\
Number & 12 & 1 & 8 & 6 & 16 & 9 & 16 & 4 & 4 & 4 \\
\hline Degree & 288 & 324 & 384 & 432 & 648 & 768 & 864 & 972 & 1152 & 1296 \\
Number & 8 & 5 & 16 & 8 & 12 & 8 & 8 & 4 & 4 & 6 \\
\hline Degree & 1458 & 1536 & 1728 & 1944 & 2304 & 2592 & 2916 & 3456 & 3888 & 5184 \\
Number & 4 & 2 & 14 & 4 & 2 & 8 & 5 & 1 & 1 & 1 \\
\hline
\end{tabular}

Table A.64: The degrees of characters in $\left.\operatorname{Irr}\left(2 \cdot 3^{1+8} \cdot 3 \cdot 2^{2} \cdot 2^{4} \cdot 3^{2} \cdot D_{8}\right) \mid \xi\right)$.

\begin{tabular}{l|rrrrrrrrrrr}
\hline Degree & 2 & 4 & 8 & 12 & 16 & 18 & 24 & 32 & 36 & 64 & 96 \\
Number & 4 & 2 & 10 & 2 & 2 & 4 & 4 & 6 & 2 & 4 & 2 \\
\hline Degree & 128 & 192 & 256 & 288 & 324 & 384 & 576 & 648 & 768 & 864 & 1152 \\
Number & 2 & 8 & 6 & 4 & 2 & 8 & 2 & 9 & 10 & 2 & 4 \\
\hline Degree & 1296 & 1536 & 1728 & 1944 & 2304 & 2592 & 2916 & 3456 & 3888 & 5184 & 5832 \\
Number & 3 & 2 & 4 & 5 & 2 & 9 & 2 & 4 & 1 & 1 & 1 \\
\hline
\end{tabular}


Table A.65: The degrees of characters in $\operatorname{Irr}\left(3^{6}:\left(2 \times L_{4}(3): 2\right): 2\right)$.

\begin{tabular}{l|rrrrrrrrr}
\hline Degree & 1 & 2 & 39 & 52 & 78 & 90 & 104 & 130 & 180 \\
Number & 4 & 1 & 4 & 8 & 1 & 4 & 1 & 4 & 1 \\
\hline Degree & 260 & 351 & 390 & 416 & 468 & 520 & 702 & 729 & 780 \\
Number & 8 & 4 & 4 & 8 & 12 & 6 & 1 & 4 & 5 \\
\hline Degree & 832 & 936 & 1040 & 1170 & 1280 & 1458 & 1560 & 2080 & 2340 \\
Number & 6 & 1 & 12 & 4 & 8 & 1 & 5 & 2 & 4 \\
\hline Degree & 2808 & 3120 & 4160 & 4680 & 7020 & 8320 & 9360 & 11232 & 12480 \\
Number & 4 & 2 & 4 & 3 & 8 & 4 & 6 & 4 & 4 \\
\hline Degree & 14040 & 16640 & 24960 & 28080 & 29952 & 33280 & 37440 & 37908 & 42120 \\
Number & 4 & 1 & 2 & 6 & 4 & 4 & 6 & 4 & 2 \\
\hline
\end{tabular}

Table A.66: The degrees of characters in $\left.\operatorname{Irr}\left(3^{6}:\left(2 \times L_{4}(3): 2\right): 2\right) \mid \xi\right)$.

\begin{tabular}{l|rrrrrrrrr}
\hline Degree & 2 & 78 & 104 & 180 & 260 & 520 & 702 & 780 & 832 \\
Number & 2 & 2 & 3 & 2 & 1 & 4 & 2 & 2 & 4 \\
\hline Degree & 936 & 1040 & 1458 & 1560 & 1664 & 2080 & 2340 & 2560 & 3120 \\
Number & 4 & 5 & 2 & 2 & 1 & 4 & 1 & 2 & 3 \\
\hline Degree & 4160 & 4680 & 5616 & 9360 & 14040 & 16640 & 18720 & 22464 & 24960 \\
Number & 4 & 4 & 1 & 2 & 2 & 2 & 1 & 1 & 3 \\
\hline Degree & 28080 & 33280 & 37440 & 42120 & 56160 & 59904 & 74880 & 75816 & \\
Number & 3 & 4 & 2 & 2 & 1 & 1 & 1 & 1 & \\
\hline
\end{tabular}

Table A.67: The degrees of characters in $\operatorname{Irr}\left(3^{6} \cdot 3^{1+4} \cdot\left(2 \times 2 S_{4}: 2\right) \cdot 2\right)$.

\begin{tabular}{l|rrrrrrrrr}
\hline Degree & 1 & 2 & 3 & 4 & 6 & 8 & 16 & 18 & 32 \\
Number & 8 & 14 & 8 & 7 & 2 & 9 & 22 & 4 & 17 \\
\hline Degree & 36 & 48 & 54 & 64 & 72 & 96 & 108 & 144 & 192 \\
Number & 17 & 4 & 4 & 4 & 19 & 9 & 9 & 11 & 4 \\
\hline Degree & 216 & 288 & 432 & 576 & 648 & 864 & 1152 & 1728 & 3456 \\
Number & 16 & 14 & 20 & 6 & 2 & 21 & 1 & 10 & 1 \\
\hline
\end{tabular}

Table A.68: The degrees of characters in $\left.\operatorname{Irr}\left(2.3^{6} .3^{1+4} .\left(2 \times 2 S_{4}: 2\right) .2\right) \mid \xi\right)$.

\begin{tabular}{l|rrrrrrrrrrr}
\hline Degree & 2 & 4 & 6 & 8 & 16 & 32 & 36 & 64 & 72 & 96 & 108 \\
Number & 4 & 6 & 4 & 2 & 4 & 14 & 2 & 6 & 11 & 2 & 2 \\
\hline Degree & 144 & 192 & 216 & 288 & 432 & 576 & 648 & 864 & 1152 & 1728 & 3456 \\
Number & 10 & 6 & 10 & 3 & 10 & 9 & 2 & 20 & 1 & 11 & 1 \\
\hline
\end{tabular}


Table A.69: The degrees of characters in $\operatorname{Irr}\left(3^{3+6} \cdot 3^{1+2} \cdot 2^{3} \cdot 2^{2}\right)$.

\begin{tabular}{l|rrrrrrrrrrrr}
\hline Degree & 1 & 2 & 4 & 6 & 8 & 12 & 16 & 18 & 24 & 36 & 48 & 54 \\
Number & 16 & 20 & 24 & 8 & 17 & 18 & 4 & 8 & 32 & 30 & 12 & 8 \\
\hline Degree & 72 & 96 & 108 & 144 & 162 & 216 & 288 & 324 & 432 & 648 & 864 & 1296 \\
Number & 25 & 2 & 18 & 8 & 8 & 42 & 1 & 10 & 12 & 16 & 1 & 2 \\
\hline
\end{tabular}

Table A.70: The degrees of characters in $\left.\operatorname{Irr}\left(2 \cdot 3^{3+6} \cdot 3^{1+2} \cdot 2^{3} \cdot 3^{2}\right) \mid \xi\right)$.

\begin{tabular}{l|rrrrrrrrrr}
\hline Degree & 2 & 4 & 8 & 12 & 16 & 24 & 36 & 48 & 72 & 96 \\
Number & 8 & 8 & 14 & 4 & 6 & 12 & 4 & 18 & 20 & 2 \\
\hline Degree & 108 & 144 & 216 & 288 & 324 & 432 & 648 & 864 & 1296 & \\
Number & 4 & 11 & 34 & 1 & 4 & 15 & 18 & 1 & 2 & \\
\hline
\end{tabular}

Table A.71: The degrees of characters in $\operatorname{Irr}(5: 4 \times \mathrm{HS}: 2)$.

\begin{tabular}{l|rrrrrrrrrr}
\hline Degree & 1 & 4 & 22 & 77 & 88 & 154 & 175 & 231 & 308 & 616 \\
Number & 8 & 2 & 8 & 8 & 2 & 8 & 8 & 8 & 6 & 2 \\
\hline Degree & 693 & 700 & 770 & 825 & 924 & 1056 & 1232 & 1386 & 1408 & 1540 \\
Number & 8 & 2 & 8 & 8 & 2 & 8 & 1 & 8 & 8 & 4 \\
\hline Degree & 1750 & 1792 & 1925 & 2520 & 2750 & 2772 & 3080 & 3200 & 3300 & 4224 \\
Number & 8 & 4 & 16 & 8 & 8 & 2 & 2 & 8 & 2 & 2 \\
\hline Degree & 5544 & 5632 & 6160 & 7000 & 7168 & 7700 & 10080 & 11000 & 12800 & \\
Number & 2 & 2 & 1 & 2 & 1 & 4 & 2 & 2 & 2 & \\
\hline
\end{tabular}

Table A.72: The degrees of characters in $\operatorname{Irr}((5: 4 \times 2 . \mathrm{HS}) .2 \mid \xi)$.

\begin{tabular}{l|rrrrrrrrrrr}
\hline Degree & 112 & 224 & 352 & 1232 & 1408 & 1848 & 2000 & 2464 & 3584 & 3696 & 3960 \\
Number & 2 & 2 & 4 & 4 & 1 & 4 & 2 & 4 & 2 & 2 & 4 \\
\hline Degree & 4000 & 4608 & 4928 & 5040 & 7168 & 7392 & 9856 & 10080 & 15840 & 18432 & \\
Number & 2 & 4 & 1 & 4 & 2 & 3 & 1 & 4 & 1 & 1 & \\
\hline
\end{tabular}

Table A.73: The degrees of characters in $\operatorname{Irr}\left(5: 4 \times 5: 4 \times S_{5}\right)$.

\begin{tabular}{l|rrrrrrrrrr}
\hline Degree & 1 & 4 & 5 & 6 & 16 & 20 & 24 & 64 & 80 & 96 \\
Number & 32 & 48 & 32 & 16 & 18 & 16 & 8 & 2 & 2 & 1 \\
\hline
\end{tabular}


Table A.74: The degrees of characters in $\operatorname{Irr}\left(2 .\left(5: 4 \times 5: 4 \times S_{5}\right) \mid \xi\right)$.

\begin{tabular}{l|rrrrrrrr}
\hline Degree & 4 & 8 & 12 & 16 & 32 & 48 & 64 & 96 \\
Number & 16 & 8 & 8 & 8 & 4 & 4 & 3 & 2 \\
\hline
\end{tabular}

Table A.75: The degrees of characters in $\operatorname{Irr}(5: 4 \times 5: 4 \times 5: 4)$.

\begin{tabular}{l|rrrr}
\hline Degree & 1 & 4 & 16 & 64 \\
Number & 64 & 48 & 12 & 1 \\
\hline
\end{tabular}

Table A.76: The degrees of characters in $\operatorname{Irr}(2 .(5: 4 \times 5: 4 \times 5: 4) \mid \xi)$.

\begin{tabular}{l|rrrrr}
\hline Degree & 2 & 4 & 8 & 16 & 64 \\
Number & 16 & 16 & 8 & 12 & 1 \\
\hline
\end{tabular}

Table A.77: The degrees of characters in $\operatorname{Irr}\left(5: 4 \times 5^{1+2} \cdot 4 . D_{8}\right)$.

\begin{tabular}{l|rrrrrrrrrrr}
\hline Degree & 1 & 2 & 4 & 8 & 16 & 20 & 32 & 40 & 64 & 80 & 160 \\
Number & 32 & 24 & 8 & 22 & 8 & 16 & 4 & 4 & 2 & 4 & 1 \\
\hline
\end{tabular}

Table A.78: The degrees of characters in $\operatorname{Irr}\left(2 .\left(5: 4 \times 5^{1+2} \cdot 4 . D_{8}\right) \mid \xi\right)$.

\begin{tabular}{l|rrrrrrrrr}
\hline Degree & 2 & 4 & 8 & 16 & 32 & 40 & 64 & 80 & 160 \\
Number & 16 & 4 & 4 & 5 & 6 & 8 & 2 & 4 & 1 \\
\hline
\end{tabular}

Table A.79: The degrees of characters in $\operatorname{Irr}\left(5^{3} \cdot L_{3}(5)\right)$.

\begin{tabular}{|c|c|c|c|c|c|c|c|c|c|c|c|c|c|c|}
\hline Degree & 1 & 30 & 31 & 96 & 124 & 125 & 155 & 186 & 620 & 1240 & 1860 & 2480 & 3100 & 3720 \\
\hline Number & 1 & 1 & 3 & 10 & 10 & 1 & 3 & 1 & 1 & 2 & 2 & 2 & 1 & 1 \\
\hline
\end{tabular}

Table A.80: The degrees of characters in $\operatorname{Irr}\left(2 \times 5^{3} \cdot L_{3}(5) \mid \xi\right)$.

\begin{tabular}{|c|c|c|c|c|c|c|c|c|c|c|c|c|c|}
\hline Degree & 130 & 31 & 96 & 124 & 125 & 155 & 186 & 620 & 1240 & 1860 & 2480 & 3100 & 3720 \\
\hline Number & 1 & 3 & 10 & 10 & 1 & 2 & 1 & 1 & 2 & 2 & 2 & 1 & \\
\hline
\end{tabular}


Table A.81: The degrees of characters in $\operatorname{Irr}\left(5_{+}^{1+4} \cdot \mathrm{GL}_{2}(5)\right)$.

\begin{tabular}{l|rrrrrrrrrrrrrr}
\hline Degree & 1 & 4 & 5 & 6 & 24 & 96 & 100 & 120 & 200 & 240 & 300 & 400 & 500 & 600 \\
Number & 4 & 10 & 4 & 6 & 4 & 1 & 1 & 4 & 2 & 4 & 2 & 2 & 1 & 1 \\
\hline
\end{tabular}

Table A.82: The degrees of characters in $\operatorname{Irr}\left(2 \times 5_{+}^{1+4} \cdot \mathrm{GL}_{2}(5) \mid \xi\right)$.

\begin{tabular}{l|rrrrrrrrrrrrrr}
\hline Degree & 1 & 4 & 5 & 6 & 24 & 96 & 100 & 120 & 200 & 240 & 300 & 400 & 500 & 600 \\
Number & 4 & 10 & 4 & 6 & 4 & 1 & 1 & 4 & 2 & 4 & 2 & 2 & 1 & 1 \\
\hline
\end{tabular}

Table A.83: The degrees of characters in $\operatorname{Irr}\left(5_{+}^{1+4} \cdot 2_{-}^{1+4} \cdot A_{5} \cdot 4\right)$.

\begin{tabular}{l|rrrrrrrrrrr}
\hline Degree & 1 & 4 & 5 & 6 & 10 & 15 & 16 & 20 & 24 & 100 & 240 \\
Number & 4 & 8 & 8 & 2 & 10 & 4 & 4 & 8 & 2 & 1 & 8 \\
\hline Degree & 300 & 384 & 400 & 480 & 500 & 1000 & 1200 & 1500 & 1536 & 1600 & 2000 \\
Number & 2 & 4 & 2 & 6 & 4 & 2 & 2 & 1 & 1 & 1 & 2 \\
\hline
\end{tabular}

Table A.84: The degrees of characters in $\operatorname{Irr}\left(2 .\left(5_{+}^{1+4} \cdot 2_{-}^{1+4} \cdot A_{5} \cdot 4\right) \mid \xi\right)$.

\begin{tabular}{l|rrrrrrrrr}
\hline Degree & 4 & 6 & 10 & 16 & 20 & 24 & 200 & 384 & 400 \\
Number & 6 & 4 & 4 & 6 & 8 & 4 & 2 & 4 & 1 \\
\hline Degree & 480 & 600 & 800 & 960 & 1000 & 1536 & 1600 & 2000 & 2400 \\
Number & 4 & 1 & 2 & 1 & 5 & 1 & 1 & 1 & 1 \\
\hline
\end{tabular}

Table A.85: The degrees of characters in $\operatorname{Irr}\left(5^{2}: 4 S_{4} \times S_{5}\right)$.

\begin{tabular}{l|rrrrrrrrrrrrrrrrr}
\hline Degree & 1 & 2 & 3 & 4 & 5 & 6 & 8 & 10 & 12 & 15 & 16 & 18 & 20 & 24 & 96 & 120 & 144 \\
Number & 8 & 12 & 8 & 12 & 8 & 4 & 12 & 12 & 14 & 8 & 4 & 4 & 4 & 10 & 8 & 8 & 4 \\
\hline
\end{tabular}

Table A.86: The degrees of characters in $\operatorname{Irr}\left(2 .\left(5^{2}: 4 S_{4} \times S_{5}\right) \mid \xi\right)$.

\begin{tabular}{l|rrrrrrrrr}
\hline Degree & 4 & 8 & 12 & 16 & 24 & 36 & 96 & 192 & 288 \\
Number & 4 & 12 & 10 & 8 & 8 & 2 & 4 & 2 & 2 \\
\hline
\end{tabular}


Table A.87: The degrees of characters in $\operatorname{Irr}\left(5^{2}: 4 S_{4} \times 5: 4\right)$.

\begin{tabular}{l|rrrrrrrrr}
\hline Degree & 1 & 2 & 3 & 4 & 8 & 12 & 16 & 24 & 96 \\
Number & 16 & 24 & 16 & 12 & 6 & 4 & 2 & 16 & 4 \\
\hline
\end{tabular}

Table A.88: The degrees of characters in $\operatorname{Irr}\left(2 .\left(5^{2}: 4 S_{4} \times 5: 4\right) \mid \xi\right)$.

\begin{tabular}{l|rrrrrrrr}
\hline Degree & 2 & 4 & 6 & 8 & 12 & 16 & 48 & 96 \\
Number & 12 & 16 & 4 & 6 & 4 & 2 & 4 & 4 \\
\hline
\end{tabular}

Table A.89: The degrees of characters in $\operatorname{Irr}\left(\left(2^{2} \times 7^{2}:\left(3 \times 2 A_{4}\right)\right): 2\right)$.

\begin{tabular}{l|rrrrrr}
\hline Degree & 1 & 2 & 3 & 4 & 6 & 48 \\
Number & 12 & 27 & 12 & 15 & 3 & 12 \\
\hline
\end{tabular}

Table A.90: The degrees of characters in $\operatorname{Irr}\left(2 .\left(2^{2} \times 7^{2}:\left(3 \times 2 A_{4}\right)\right): 2 \mid \xi\right)$.

\begin{tabular}{l|rrrrr}
\hline Degree & 2 & 4 & 6 & 8 & 96 \\
Number & 6 & 9 & 6 & 3 & 3 \\
\hline
\end{tabular}

\section{References}

1. J. L. Alperin, 'Weights for finite groups', The Arcata Conference on Representations of Finite Groups, Proc. Sympos. Pure Math. 47 (1987) 369-379. 121

2. JiAnbei An and C. W. EAton, 'The $p$-local rank of a block', J. Group Theory 3 (2000) 369-380. 121, 123

3. JiAnbei An and C. W. EAton, 'Modular representation theory of blocks with trivial intersection defect groups', Algebr. Represent. Theory, to appear. 126, 138

4. JiAnbei An and E. A. O'Brien, 'A local strategy to decide the Alperin and Dade conjectures', J. Algebra 206 (1998) 183-207. 120, 125, 126

5. JiAnbei An and E. A. O'Brien, 'The Alperin and Dade conjectures for the simple Fischer group $\mathrm{Fi}_{23}$ ', International J. Algebra Comput. 9 (1999) 621-670. 120, 125

6. Wieb Bosma, John Cannon and Catherine Playoust, 'The Magma algebra system I: the user language', J. Symbolic Comput. 24 (1997) 235-265. 120

7. J. H. Conway, R. T. Curtis, S. P. Norton, R. A. Parker and R. A. Wilson, Atlas of finite groups (Clarendon Press, Oxford, 1985). 123, 131, 132

8. E. C. DADE, 'Counting characters in blocks', I, Invent. Math. 109 (1992) 187-210. 121,126 
9. E. C. DADE, 'Counting characters in blocks', II.9, Representation theory of finite groups (Columbus, OH, 1995), Ohio State Univ. Math. Res. Inst. Publ. 6 (de Gruyter, Berlin, 1997) 45-59. 120, 121

10. The GAP Team, 'GAP - Groups, Algorithms, and Programming', Version 4. Lehrstuhl D für Mathematik, RWTH Aachen, and School of Mathematical and Computational Sciences, University of St Andrews (2000). 120, 125

11. G. Hiss and K. Lux, Brauer trees of sporadic groups (Oxford Science Publications, 1989). 131, 132

12. I. M. IsAaCS and G. NAVARRO, 'New refinements of the McKay conjecture for arbitrary finite groups', Ann of Math 156 (2002) 333-344. 120, 122

13. R. KNÖRR, 'On the vertices of irreducible modules', Ann. of Math. 110 (1979) 487-499. 138

14. S. Linton, R. Parker, P. Walsh and R. Wilson, 'Computer construction of the Monster', J. Group Theory 1 (1998) 307-337. 124, 125

15. K. Uno, 'Conjectures on character degrees for the simple Thompson group', Osaka J. Math., to appear. 120, 122

16. Robert A. Wilson, 'Some subgroups of baby monster', Invent. Math. 89 (1987) 197-218. 120, 125, 127, 128, 129, 130

17. R. A. WILSON, et al., 'ATLAS of finite group representations', http: //www.mat.bham.ac.uk/atlas. 124, 125

Jianbei An an@math.auckland.ac.nz

Department of Mathematics

University of Auckland

Auckland

New Zealand

R. A. Wilson R.A.Wilson@bham.ac.uk

Department of Mathematics

The University of Birmingham

Birmingham B15 2TT

United Kingdom 